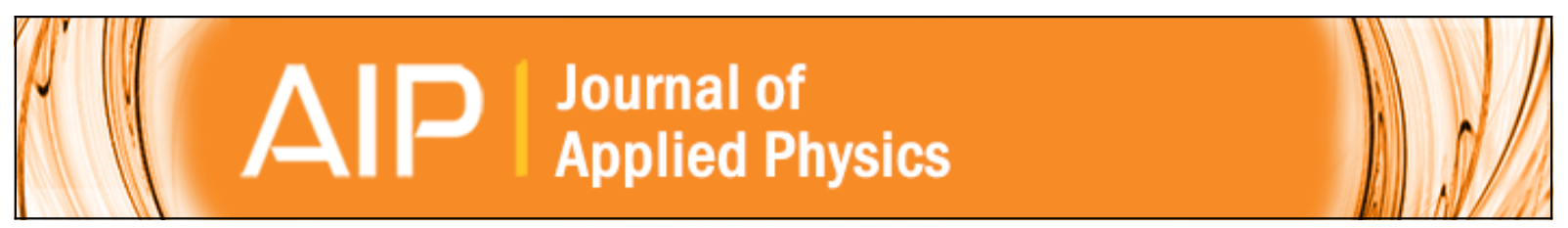

\title{
Making use of x-ray optical effects in photoelectron-, Auger electron-, and x-ray emission spectroscopies: Total reflection, standing-wave excitation, and resonant effects
}

\author{
S.-H. Yang, A. X. Gray, A. M. Kaiser, B. S. Mun, B. C. Sell, J. B. Kortright, and C. S. Fadley
}

Citation: Journal of Applied Physics 113, 073513 (2013); doi: 10.1063/1.4790171

View online: http://dx.doi.org/10.1063/1.4790171

View Table of Contents: http://scitation.aip.org/content/aip/journal/jap/113/7?ver=pdfcov

Published by the AIP Publishing

\section{Articles you may be interested in}

X-ray Spontaneous Emission Control By 1D-PBG Structure

AIP Conf. Proc. 1221, 51 (2010); 10.1063/1.3399256

Determination of buried interface composition and magnetism profiles using standing-wave excited soft x-ray emission and inelastic scattering

J. Appl. Phys. 103, 083515 (2008); 10.1063/1.2906331

Chemical bonding state analysis of silicon carbide layers in $\mathrm{Mo} / \mathrm{SiC} / \mathrm{Si}$ multilayer mirrors by soft x-ray emission and absorption spectroscopy

Appl. Phys. Lett. 77, 2653 (2000); 10.1063/1.1318231

Effect of rapid thermal annealing temperature on the formation of CoSi studied by $x$-ray photoelectron spectroscopy and micro-Raman spectroscopy

J. Vac. Sci. Technol. A 18, 1690 (2000); 10.1116/1.582408

Mo/SiO 2 multilayers for soft x-ray optical applications

J. Appl. Phys. 85, 3175 (1999); 10.1063/1.369657

Frustrated by

old technology?

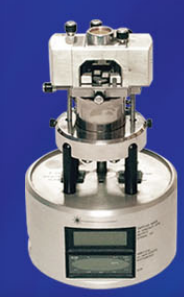

Is your AFM dead

and can't be repaired?

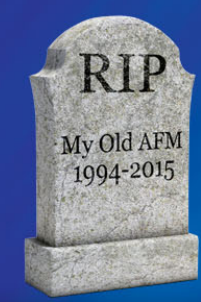

Sick of bad customer support?

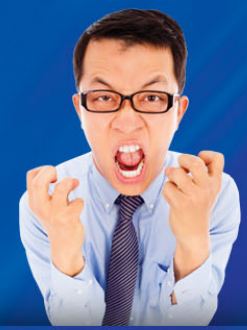

It is time to upgrade your AFM Minimum $\$ 20,000$ trade-in discount for purchases before August 31st

Asylum Research is today's technology leader in AFM

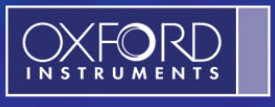




\title{
Making use of x-ray optical effects in photoelectron-, Auger electron-, and $x$-ray emission spectroscopies: Total reflection, standing-wave excitation, and resonant effects
}

\author{
S.-H. Yang, ${ }^{1}$ A. X. Gray, ${ }^{2,3,4}$ A. M. Kaiser, ${ }^{2,3,5}$ B. S. Mun, ${ }^{6,7}$ B. C. Sell, ${ }^{2,3,8, a)}$ J. B. Kortright, ${ }^{2}$ \\ and C. S. Fadley ${ }^{2,3}$ \\ ${ }^{1}$ IBM Almaden Research Center, San Jose, California 95120, USA \\ ${ }^{2}$ Materials Sciences Division, Lawrence Berkeley National Laboratory, Berkeley, California 94740, USA \\ ${ }^{3}$ Department of Physics, University of California at Davis, Davis, California 95616, USA \\ ${ }^{4}$ Stanford Institute for Materials and Energy Science, Stanford University and SLAC National Accelerator \\ Laboratory, Menlo Park, California 94025, USA \\ ${ }^{5}$ Peter Grunberg Institute, PGI-6, Forschungszentrum Jülich, 52425 Jülich, Germany \\ ${ }^{6}$ Advanced Light Source, Lawrence Berkeley National Laboratory, Berkeley, California 94720, USA \\ ${ }^{7}$ Department of Applied Physics, Hanyang University, Ansan, Gyeonggi 426-791, South Korea \\ ${ }^{8}$ Department of Physics, Otterbein College, Westerville, Ohio 43081, USA
}

(Received 2 August 2012; accepted 15 January 2013; published online 21 February 2013)

\begin{abstract}
We present a general theoretical methodology and related open-access computer program for carrying out the calculation of photoelectron, Auger electron, and x-ray emission intensities in the presence of several x-ray optical effects, including total reflection at grazing incidence, excitation with standing-waves produced by reflection from synthetic multilayers and at core-level resonance conditions, and the use of variable polarization to produce magnetic circular dichroism. Calculations illustrating all of these effects are presented, including in some cases comparisons to experimental results. Sample types include both semi-infinite flat surfaces and arbitrary multilayer configurations, with interdiffusion/roughness at their interfaces. These x-ray optical effects can significantly alter observed photoelectron, Auger, and x-ray intensities, and in fact lead to several generally useful techniques for enhancing surface and buried-layer sensitivity, including layer-resolved densities of states and depth profiles of element-specific magnetization. The computer program used in this study should thus be useful for a broad range of studies in which x-ray optical effects are involved or are to be exploited in next-generation surface and interface studies of nanoscale systems. (C) 2013 American Institute of Physics. [http://dx.doi.org/10.1063/1.4790171]
\end{abstract}

\section{INTRODUCTION}

Emission spectroscopies excited with soft or hard x-rays from synchrotron sources constitute some of the most powerful tools for probing atomic structure, electronic structure, and magnetic properties of bulk materials, as well as surfaces, buried layers, and the interfaces between layers. In the case of photoelectron emission, if the excitation is in the soft $\mathrm{x}$-ray regime of $c a \cdot 100-1000 \mathrm{eV}$, the short inelastic mean free paths (IMFPs) of $\sim 5-25 \AA$ (Ref. 1) have led to many investigations of chemical and physical properties at surfaces or layers that are close to surfaces, but also with sensitivity to bulk properties. The techniques of $\mathrm{x}$-ray photoelectron spectroscopy (XPS) ${ }^{2}$ and Auger electron spectroscopy $(\mathrm{AES})^{3}$ are both in this category. More recently, there is growing interest in carrying out photoemission in the multi$\mathrm{keV}$ regime, yielding hard x-ray photoemission (HXPS, HAXPES), for which the IMFPs can be $50-150 \AA$, implying much more bulk and buried layer/buried interface sensitivity. ${ }^{4,5}$ Alternatively, $\mathrm{x}$-ray emission (XES) spectroscopy and its close relative resonant inelastic $\mathrm{x}$-ray scattering (RIXS) ${ }^{6}$ and $\mathrm{x}$-ray absorption (XAS) spectroscopy based on total electron yield ${ }^{7}$ are more bulk-sensitive since the attenuation

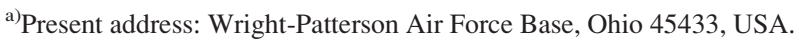

lengths for emitted photons or the creation of secondary electrons are significantly larger. Furthermore, XPS, AES, and XES/RIXS are particularly versatile since they are elementand orbital-specific techniques. We will here focus primarily on XPS and XES/RIXS, commenting to some degree also on AES, and will consider the calculations of various X-ray optical effects that can strongly modify the total electric field (incident plus reflected) vs. depth inside of samples, thus affecting spectral intensities: total reflection at grazing incidence, excitation with standing-waves produced by reflection from synthetic multilayers, ${ }^{8}$ and perhaps also at core-level resonance conditions, and the use of variable polarization to produce magnetic circular or other dichroism effects.

X-ray optical effects in XPS were first discussed theoretically and experimentally with regard to going into total reflection for a homogeneous semi-infinite sample by Henke, ${ }^{9}$ and it was soon demonstrated that these effects could be used to enhance surface sensitivity. ${ }^{10}$ Somewhat later, the theory of x-ray optical effects in spectroscopy was further discussed, ${ }^{11}$ and total reflection was more systematically developed as a tool for enhancing surface sensitivity and reducing inelastic backgrounds, leading to what has been termed grazingincidence XPS (GIXPS) ${ }^{12}$ or total reflections XPS (TRXPS). ${ }^{13}$

Beyond these total reflection studies, x-ray optical effects have not been rigorously accounted for in the analysis 
of most photoemission or x-ray emission data, with the assumption being made that the $\mathrm{x}$-rays penetrate much more deeply, and essentially without attenuation or modulation, into the sample, as compared to the depths from which the photoelectrons or fluorescent $\mathrm{x}$-rays are emitted. Yet x-ray optical effects can play a particularly significant role for measurements done at grazing incidence angles, ${ }^{9-13}$ near absorption-edge resonances, ${ }^{14}$ in Bragg-like reflection from single-crystal planes ${ }^{15-17}$ and synthetic multilayers, ${ }^{8,18-32}$ and with variable polarization so as to produce magnetic and other dichroic effects. ${ }^{19,21,30}$

In beginning this discussion, it is worthwhile to discuss in more detail the specific types of $\mathrm{x}$-ray optical effects that need to be considered: first are optical interference effects that modify the total electric field intensity vs. depth into the sample. These result directly from the consideration of electromagnetic wave propagation into and out of samples (as developed in Sec. II of this work), and include effects near the critical angle in semi-infinite samples, as well as interference effects in layered films. They all result from the phase and amplitude of the upward propagating (reflected) wave field relative to the incident (downward propagating) wave field, as given by the sum of these 2 fields as a function of depth, which we take to be the $z$ coordinate. Second, tuning the photon energy across core resonances can modify these interference effects markedly, thus changing the behavior of total wave field vs. $z$. Third, varying the polarization of the radiation, either at a non-resonant or a resonant energy, can further alter intensities and the variation of the total wave field vs. $z$.

While these optical processes can in one sense be viewed as complicating the analysis of the experimental data in many cases, the effects they produce also carry valuable information related to the structural, optical, and electronic properties of the sample. For example, one more recent approach exploits $\mathrm{x}$-ray optical effects with high-contrast $\mathrm{x}$-ray standing waves (SWs) that are artificially generated by reflection from a nm-scale multilayer mirror to probe buried interfaces via XPS ${ }^{18,21}$ and XAS. ${ }^{19}$ The SW XPS technique has in fact been found to possess several advantages over other methods for probing buried layers and interfaces: it is non-destructive and element-specific, has a high depthresolution estimated to be ca. $2-3 \AA$, and it can be used in various modalities including with $\mathrm{X}$-ray magnetic circular and linear dichroism (XMCD and XMLD) and in a photoelectron microscope to add a third depth dimension. ${ }^{19,27,28}$

In all of the above examples, a complete understanding and exhaustive theoretical consideration of the interaction between the radiation and the relevant solid state materials and/or nanostructures are essential in order to make quantitative use of experimental data. As one specific example, the deconvolution of spectra so as to derive surface and bulk contributions has been a controversial issue in resonant $\mathrm{x}$-ray photoemission spectroscopy (RPES) for cerium-based strongly correlated systems. ${ }^{33-37}$ In this particular case, it has been recognized that the notion of X-ray optics is essential to understand high-resolution RPES more accurately and quantitatively because the photon energy is scanned through the absorption edges of cerium, with the x-ray penetration depth changing dramatically during such a scan. Another specific example of where modeling of x-ray optical effects is essential to understand the system is the characterization of multilayer film stacks and superlattices, which are ubiquitous in semiconductor technology, spintronic applications, and various energy conversion devices. For example, major efforts in the past decades have been devoted to the studies of magnetic multilayer film stacks ${ }^{21,38}$ and superlattice mirrors used in EUV lithography, ${ }^{18,39}$ where consideration of $\mathrm{x}$ ray optical effects is essential to extract physical information about the structure and properties of the sample from spectroscopic measurements. Other driving forces for more quantitatively dealing with $\mathrm{x}$-ray optics in spectroscopy are the development of new state-of-the-art technologies involving complex nanostructures and the continued improvement of high-brilliance and sub-micron spot synchrotron radiation sources. Thus, allowance for $\mathrm{x}$-ray optics in spectroscopy is becoming increasingly more important as a tool for research, characterization, and metrology.

In this paper, we thus present a general discussion of $x$-ray optics, together with the emission processes for photoelectrons, Auger electrons, and fluorescent x-rays. These are incorporated into a versatile public-access computer program so as to provide for more accurate and quantitative analysis of XPS and XES/RIXS, as well as AES, spectra. Section II discusses the theoretical details of the underlying physics describing the interaction between the solid sample, the exciting X-ray wavefields (including both incident and reflected), and the emitted waves (electrons or fluorescent photons). The wavefields of the emitted photons also may scatter from layers in the sample. The excitation of any of these incoherent emission processes is proportional to the total wavefield intensity at a given point, rather than its amplitude. The downloadable program is available elsewhere, together with instructions for its use. ${ }^{40}$ Section III presents several illustrative applications to the analysis of emission from semiinfinite systems, soft $\mathrm{x}$-ray mirrors (also used as standing wave generators), resonant excitation through core absorption edges, and measurements with variable polarization. Section IV summarizes these results and presents an outlook for further studies and improvements in theoretical modeling.

\section{BASIC THEORETICAL FORMALISM}

We here present the basic theoretical components that are included in what we term the Yang X-ray optics (YXRO) computer program, ${ }^{40}$ beginning with the fundamental relations based on Maxwell's Equations, and then incorporating the excitation and emission processes for photoelectrons, Auger electrons, and fluorescent $\mathrm{x}$-rays so as to yield expressions that can be applied to a simple semi-infinite material, as well as to an arbitrary multilayer structure, with intermixing/interdiffusion at all interfaces, and the possibility of simulating dichroism effects. At the outset, we note that this program has been tested extensively and used to successfully model a variety of sets of experimental data, to which we refer the reader. ${ }^{18,20-32}$ We here thus focus primarily on the theoretical modeling of various x-ray optical effects, 
pointing out the actual and potential utility of them in future studies of buried layers and interfaces.

We begin in Figs. 1 and 2 by defining various important physical quantities for describing the interaction of the radiation with a semi-infinite sample, and an arbitrary multilayer sample, respectively. Specifically, in Fig. 1, medium 1 is vacuum with index of refraction $n_{1}$ equal to unity, and medium 2 is a semi-infinite substrate with index of refraction $n_{2}=1-\delta+i \beta$. Although it has been pointed out that using this scalar index of refraction approximation does not rigorously describe the inhomogeneous nature of wave fields in absorbing media, ${ }^{41}$ this approximation has been used successfully to interpret experimental photoemission data previously, e.g., by Henke ${ }^{9}$ and is expected to be valid for soft $\mathrm{x}$-ray energies where $|\beta| \ll 1-\delta .{ }^{11}$ The electric field, which can be involved with various types of polarization, including linear and circular, is $\vec{E}_{1}$ for the incident wave, and $\vec{E}_{1}^{\prime}$ for the reflected wave, with wave vectors of propagation of $\vec{k}_{1}$ and $\vec{k}_{1}^{\prime}$, respectively; both the $s$ and $p$ polarizations are shown in this figure. The incidence angle relative to the surface is $\phi_{i n c}$ as measured with respect to the surface, or the complement of this angle $\theta_{\text {inc }}$ as measured with respect to the surface normal. Simple geometric considerations in specular reflection dictate that the period of the standing wave electric field intensity $|\vec{E}|^{2}$ is given by $\lambda_{S W}=\lambda_{x} /\left(2 \sin \phi_{\text {inc }}\right)$. The coordinate $z$ represents depth into medium 2 . The incidence and specular reflection angle is defined relative to the surface normal as $\theta_{\text {inc }}$, and the refracted angle inside medium 2 is $\theta_{2}$; again defined relative to the surface normal. In fact, for x-rays with index of refraction less than unity, $\theta_{2}$ is greater than $\theta_{i n c}$, and thus not as shown in Fig. $1 . \overrightarrow{k_{2}}$ is the wave vector of the transmitted radiation moving downward in medium 2 with electric field $\vec{E}_{2}=E_{2} \hat{e}$ (with $\hat{e}$ a unit

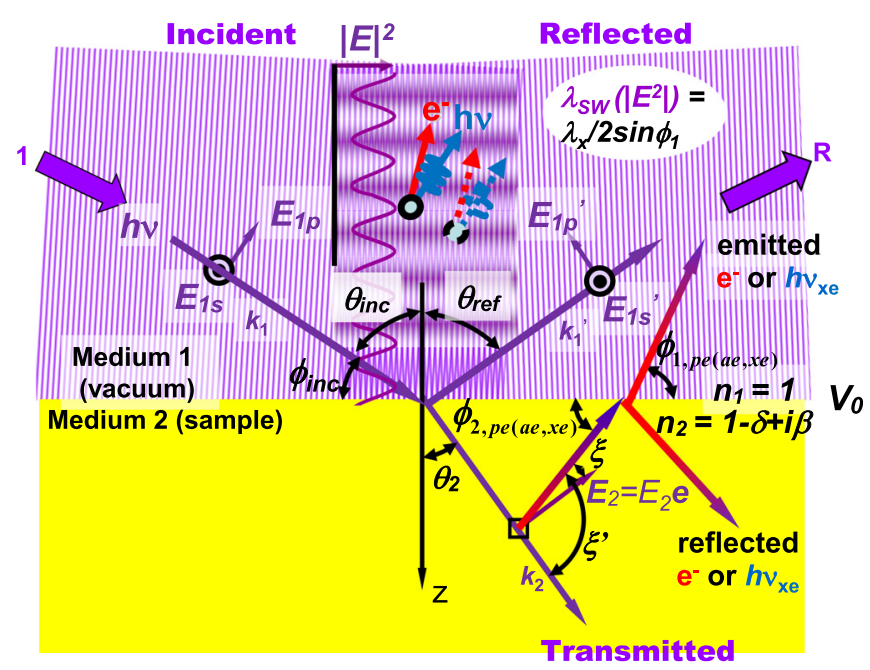

FIG. 1. Schematic diagram of the x-ray optical effects and spectroscopic processes for emission of photoelectrons (pe), Auger electrons (ae), or $\mathrm{x}$-rays (xe) from a semi-infinite medium, with various symbols defined. Here, the $\mathrm{x}$-rays are incident from medium $1=$ vacuum on the surface of medium 2, with index of refraction $n_{2}=1-\delta+i \beta$. Incident, reflected, and transmitted wave are shown, together with various angles to quantitatively model the overall processes. Also shown is a schematic illustration of how the emission of an electron or an x-ray from an atom changes in relative intensity depending on its position with respect to the maxima and minima of the standing wave, with the relative magnitude of $|E|^{2}$ also indicated.

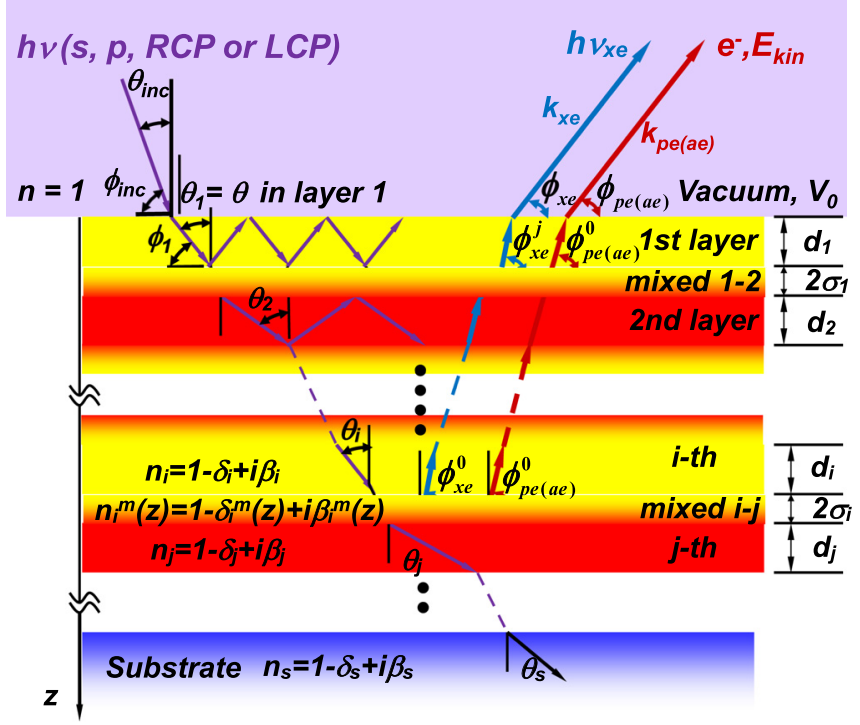

FIG. 2. Schematic diagram of the $\mathrm{x}$-ray optical effects and spectroscopic processes for a general multilayer sample, including roughness/interdiffusion at interfaces and with various symbols defined.

vector) and $\xi$ and $\xi^{\prime}$ are angles of emission of photoelectrons (pe), Auger electrons (ae), or x-rays (xe) relative to $\vec{E}_{2}$ or $\vec{k}_{2}$, respectively. The emission angles $\phi_{2, p e(a e, x e)}$ in medium 2 , and finally into vacuum $\phi_{1, p e(a e, x e)}$ are defined relative to the relevant surface/interface between the two media. X-ray incidence and refraction/reflection angles $\theta$ will generally be defined from the surface normal, but for emission processes the angles $\phi$ will be defined from the surface or the relevant buried interface. We will follow this convention for angles of incidence/reflection and emission in the more general $\mathrm{x}$-ray optical discussion for a multilayer sample below. However, in describing Bragg reflection from multilayers, as well as $\mathrm{X}$-ray attenuation at incidence, it is more intuitive to define the $\mathrm{x}$-ray incidence angle relative to the surface, as we have done above in the Bragg equation and will also do in some equations below.

In Fig. 2, this picture is generalized to describe an arbitrary multilayer sample, in which the layers are labeled with indices $1,2, \ldots ., \mathrm{j}$, as deposited on a semi-infinite substrate. Each layer has its unique index of refraction, and the computer program to be described later permits including the effects of intermixing, or equivalently interface roughness, at each interface. As illustrated here, the sample itself would be a periodic multilayer composed of repeating bilayers of 1-2, $3-4$, etc., but the program can in fact deal with any configuration of layers. Note also that multiple scattering of the x-rays within all layers is allowed for, using the Parratt algorithm. ${ }^{42}$ By contrast, the photoelectrons are assumed to travel in straight-line trajectories to the top surface of the sample. However, the photoelectrons may be refracted and reflected in crossing the inner potential barrier $V_{0}$ at the surface, ${ }^{43}$ as schematically indicated in Figs. 1 and 2. The YXRO program is thus complementary to the SESSA program for XPS surface analysis, ${ }^{44,45}$ in that the latter allows for atomic elastic scattering effects inside the sample as the photoelectrons exit, but includes neither any effects of X-ray optics nor refraction at the surface barrier, which are included in YXRO. 


\section{A. A semi-infinite sample}

When a plane electromagnetic wave originating from vacuum (coordinate $z<0$, dielectric constant $\varepsilon_{0}=1$ ) is incident onto an idealized mirror-like flat surface $(z=0)$ of a solid $\left(z>0\right.$, with dielectric constant $\left.\varepsilon=(1-\delta+i \beta)^{2}\right){ }^{46}$ linear optics based on Maxwell's equations yields spatially and temporally dependent electric fields for incoming $\left(\mathbf{E}_{1}(\mathbf{r}, t ; h \nu)\right)$, reflected $\left(\mathbf{E}_{1}^{\prime}(\mathbf{r}, t ; h \nu)\right)$, and transmitted $\left(\mathbf{E}_{2}(\mathbf{r}, t ; h \nu)\right.$ ) waves at the $z=0$ interface as follows (see, Fig. 1)

$$
\begin{aligned}
& \mathbf{E}_{1}(\mathbf{r}, t ; h \nu)=\mathbf{E}_{1}\left(z=0^{-}\right) e^{i\left(\mathbf{k}_{1} \cdot \mathbf{r}-\omega t\right)}, \\
& \mathbf{E}_{1}^{\prime}(\mathbf{r}, t ; h \nu)=\mathbf{E}_{1}^{\prime}\left(z=0^{-}\right) e^{i\left(\mathbf{k}_{1}^{\prime} \cdot \mathbf{r}-\omega t\right)}, \\
& \mathbf{E}_{2}(\mathbf{r}, t ; h \nu)=\mathbf{E}_{2}\left(z=0^{+}\right) e^{i\left(\mathbf{k}_{2} \cdot \mathbf{r}-\omega t\right)} .
\end{aligned}
$$

Applying the appropriate boundary conditions yields Snell's law, ${ }^{47}$

$$
n_{1} \sin \theta_{1}=n_{1}^{\prime} \sin \theta_{1}^{\prime}=n_{2} \sin \theta_{2} .
$$

The special condition $n_{1}=n_{1}^{\prime}$ results in $\theta_{1}=\theta_{1}^{\prime}$. In general, all media can be considered to be conducting in the soft and hard x-ray regimes of frequencies, and this implies that the angle $\theta_{2}$ is complex because $n_{2}$ is complex and with a nonzero absorbing component $\beta$. The wave propagation in any medium can thus be expressed in terms of incident electromagnetic waves via these boundary conditions. That is, if it is assumed that the magnitudes of reflected and transmitted waves become, respectively,

$$
\begin{aligned}
& E_{1}^{\prime}\left(z=0^{-}\right)=r E_{1}\left(z=0^{-}\right), \\
& E_{2}\left(z=0^{+}\right)=t E_{1}\left(z=0^{-}\right),
\end{aligned}
$$

where $r$ and $t$ represent reflection and transmission, respectively, then with the boundary conditions on the field components tangential to the surface $\mathbf{E}_{1 t}+\mathbf{E}_{1 t}^{\prime}=\mathbf{E}_{2 t}$ and $\mathbf{B}_{1 t}+\mathbf{B}_{1 t}^{\prime}=\mathbf{B}_{2 t}$, we obtain the relevant Fresnel coefficients for $\mathrm{s}$ and $\mathrm{p}$ polarized radiation, with the usual definitions of $\mathrm{s}$ polarization perpendicular to the planes of Figs. 1 and 2, and $p$ polarization in these planes,

$$
\begin{aligned}
r^{s} & =\frac{n_{1} \cos \theta_{1}-n_{2} \cos \theta_{2}}{n_{1} \cos \theta_{1}+n_{2} \cos \theta_{2}}, \\
t^{s} & =\frac{2 n_{1} \cos \theta_{1}}{n_{1} \cos \theta_{1}+n_{2} \cos \theta_{2}}(\mathrm{~s}-\text { polarization }), \\
r^{p} & =\frac{n_{1} \cos \theta_{2}-n_{2} \cos \theta_{1}}{n_{1} \cos \theta_{2}+n_{2} \cos \theta_{1}}, \\
t^{p} & =\frac{2 n_{1} \cos \theta_{1}}{n_{1} \cos \theta_{2}+n_{2} \cos \theta_{1}}(\mathrm{p}-\text { polarization }) .
\end{aligned}
$$

The transmitted electric field strength $E_{2}(\mathbf{r}, t)$ can then be written as

$$
\begin{aligned}
E_{2}(\mathbf{r}, t ; h \nu) & =E_{2}\left(z=0^{+}\right) e^{i\left(\mathbf{k}_{2} \cdot \mathbf{r}-\omega t\right)} \\
& =t E_{1}\left(z=0^{-}\right) e^{i\left(k_{2} x \sin \theta_{2}+k_{2} z \cos \theta_{2}-\omega t\right)} .
\end{aligned}
$$

Since medium 1 is vacuum $\left(n_{1}=1\right)$, Eq. (2) becomes

$$
k_{1} \sin \theta_{1}=k_{2} \sin \theta_{2}=k_{2}^{r} \sin \theta_{2}=\text { Real number. }
$$

Here, $\mathbf{k}_{2}=\mathbf{k}_{2}^{r}+i \mathbf{k}_{2}^{i}, \mathbf{k}_{2}^{r}=\operatorname{Re}\left(\mathbf{k}_{2}\right), \mathbf{k}_{2}^{i}=\operatorname{Im}\left(\mathbf{k}_{2}\right)$, and $\mathbf{k}_{2}=k_{2} \sin \theta_{2}$ $\hat{\mathbf{x}}+k_{2} \cos \theta_{2} \hat{\mathbf{z}} \cdot k_{2}$ and $\cos \theta_{2}$ are complex while $k_{1}, k_{2}^{r}, k_{2}^{i}$, and $\sin \theta_{2}$ are real. Equation (6) implies that $\mathbf{k}_{2}^{i}$ has only a normal (z-) component and $\mathbf{k}_{2}=k_{2}^{r} \sin \theta_{2} \hat{\mathbf{x}}+k_{2}^{r} \cos \theta_{2} \hat{\mathbf{z}}+i k_{2}^{i} \hat{\mathbf{z}}=k_{1}$ $\sin \theta_{1} \hat{\mathbf{x}}+\left(k_{2}^{r} \cos \theta_{2}+i k_{2}^{i}\right) \hat{\mathbf{z}}$. Hence $\mathbf{k}_{2}=k_{2}^{r} \sin \theta_{2} \hat{\mathbf{x}}+k_{2}^{r} \cos \theta_{2}$ $\hat{\mathbf{z}}+i k_{2}^{i} \hat{\mathbf{z}}=k_{1} \sin \theta_{1} \hat{\mathbf{x}}+\left(k_{2}^{r} \cos \theta_{2}+i k_{2}^{i}\right) \hat{\mathbf{z}} \quad$ and $\quad k_{2}^{r} \cos \theta_{2}+i k_{2}^{i}$ $=k_{2} \cos \theta_{2}$. Then, the electric field strength in medium 2 can be described as

$E_{2}(\mathbf{r}, t ; h \nu)=t E_{1}\left(z=0^{-}\right) e^{i\left(k_{2} x \sin \theta_{2}+\operatorname{Re}\left\{k_{2} z \cos \theta_{2}\right\}+i \operatorname{Im}\left\{k_{2} z \cos \theta_{2}\right\}-\omega t\right)}$.

The electric field strength in the medium needs to be normalized with respect to the incoming wave, i.e., $\left|E_{1}\left(z=0^{-}\right)\right|=1$, thus the squared electric field strength in media 2 becomes

$$
I(z)=\left|E_{2}(z)\right|^{2}=|t|^{2} e^{-2 \operatorname{Im}\left\{k_{2} z \cos \theta_{2}\right\}} .
$$

The photon energy is transported in the form of the Poynting vector $\mathbf{S}=\mathbf{E} \times \mathbf{B}$ and, therefore, $\mathbf{S} \propto|\mathbf{E}|^{2}$. Using Eq. (8), the differential spectral intensities for photoelectron, Auger electron, and $\mathrm{x}$-ray emission, emanating from level $k$ in an atom of type $A$ at depth $z$, as excited by a photon of energy $h \nu$ and polarization $\hat{\varepsilon}$ into a direction $\phi_{p e(a e, x e)}^{0}$, can be written as

$$
\begin{aligned}
& \frac{d I_{A k, p e}\left(z ; \hat{\varepsilon}, h \nu, \phi_{p e}^{0}\right)}{d z}= \rho_{A} \frac{d \sigma_{p e}^{A k}(h \nu, \hat{\varepsilon})}{d \Omega}\left|E_{2}(z ; h \nu)\right|^{2} \\
& \times\left[1-R\left(\phi_{p e}^{0}\right)\right] e^{-\frac{z}{\Lambda_{p e} \sin \phi_{p e}^{0}}} \\
&(\text { photoemission }), \\
& \frac{d I_{A k, a e}\left(z ; \hat{\varepsilon}, h \nu, \phi_{a e}^{0}\right)}{d z}=\rho_{A} \frac{d \sigma_{a e}^{A k}(h \nu, \hat{\varepsilon})}{d \Omega}\left|E_{2}(z ; h \nu)\right|^{2} \\
& \times\left[1-R\left(\phi_{a e}^{0}\right)\right] e^{-\frac{z}{\Lambda_{a e} \sin \phi_{a e}^{0}}}(\text { Auger }), \\
& \frac{d I_{A k, x e}\left(z ; \hat{\varepsilon}, h \nu, \phi_{1 x e}^{0}\right)}{d z}= \rho_{A} \frac{d \sigma_{x e}^{A k}(h \nu, \hat{\varepsilon})}{d \Omega}\left|E_{2}(z ; h \nu)\right|^{2} \\
& \times \mid E_{1 x e}\left(z ; h \nu_{x e},\left.\phi_{1 x e}^{0}\right|^{2}\right. \\
&(\mathrm{x}-\text { ray emission }),
\end{aligned}
$$

where $\rho_{\mathrm{A}}$ is the density of atom $\mathrm{A}, k$ labels the subshell that is initially excited to cause emission of a photoelectron, or a secondary Auger electron or x-ray (and is here not a wave vector), the angular variables $\phi_{p e}^{0}, \phi_{a e}^{0}$, and $\phi_{1 x e}^{0}$ describe the initial emission direction at depth $z$, the differential atomic cross sections $\frac{d \sigma_{p e(a e, x)}^{A k}(h \nu, \hat{\varepsilon})}{d \Omega}$ describe the probability of exciting each type of emission into a given initial direction, $\Lambda_{\text {pe(ae) }}$ are the inelastic mean free paths of electrons, or more correctly effective attenuation lengths (EALs) if allowance is made for elastic scattering effects as well, with the EALs being estimated using for example, the TPP-2 M method of Tanuma, Powell, and Penn ${ }^{48}$ or derived directly from prior experimental data for many materials. ${ }^{45}$ For photoelectron 
emission and in the dipole approximation, $\frac{d \sigma_{p e}^{A k}(h \nu, \hat{\varepsilon})}{d \Omega}$ can be expressed in the usual way as a function of the polarization of the incident radiation via the asymmetric parameter $\eta_{A k}$ for the $A k$ subshell, the angle between the polarization direction and the electron emission direction $\xi$ (for linearly polarized light), which determines also $\phi_{p e}^{0}$, or $\xi^{\prime}$, the angle between $\mathrm{x}$-ray propagation direction $\hat{k}_{2}$ and electron emission direction (for unpolarized light). It should be noted that the actual take-off angle $\phi_{p e(a e)}$ from the surface to the detector is different from the angle $\phi_{p e(a e)}^{0}$ at which the electron leaves a given atom due to the electron refraction at the inner potential surface barrier. Also, the back reflection of outgoing electrons at this barrier should be taken into account for complete generality; this we describe by $R\left(\phi_{p e}^{0}\right)$, and the two relevant quantities can readily be obtained from $\phi_{p e(a e)}=\cos ^{-1}\left[\left(1+V_{0} / E_{k i n}\right)^{1 / 2} \cos \phi_{p e(a e)}^{0}\right]$ and $R\left(\phi_{p e(a e)}^{0}\right)$ $=\left[\frac{1-\left(1-V_{0} / E_{k i n} \sin ^{2} \phi_{p e(a e)}^{0}\right)^{1 / 2}}{1+\left(1-V_{0} / E_{k i n} \sin ^{2} \phi_{p e(a e)}^{0}\right)^{1 / 2}}\right]^{2}$, where $V_{0}$ is the inner potential and $E_{k i n}$ is the electron kinetic energy. Similar refraction and back reflection of the emitted x-rays can also occur at the surface. The differential cross sections $\frac{d \sigma_{a e(x e)}^{A k}(h \nu, \hat{\varepsilon})}{d \Omega}$ for Auger or x-ray emission are more complex in nature, and we will not deal with them quantitatively here but we note that the depth dependence of both will be controlled by $\left|E_{2}(z ; h \nu)\right|^{2}$ in Eqs. (9) above.

In the dipole approximation, and for given $\eta_{A k}, \xi$, and $\xi^{\prime}$ values, Eq. (9a) for photoemission from a semi-infinite sample becomes for polarized radiation,

$$
\begin{aligned}
\frac{d I_{A k, p e}\left(z ; h v, \hat{\varepsilon}, \phi_{p e}^{0}\right)}{d z}= & \rho_{A} \frac{\sigma_{p e}^{A k}(h \nu)}{4 \pi}\left[1+\eta_{A k}\left(\frac{3}{2} \cos ^{2} \xi-\frac{1}{2}\right)\right] \\
& \times\left|E_{2}(z ; h v)\right|^{2}\left[1-R\left(\phi_{p e}^{0}\right)\right] e^{-\frac{z}{\Lambda_{p e} \sin \phi_{p e}^{0}}}
\end{aligned}
$$

and for unpolarized radiation,

$$
\begin{aligned}
\frac{d I_{A k, p e}\left(z ; h v, \hat{k}_{x}, \phi_{p e}^{0}\right)}{d z}= & \rho_{A} \frac{\sigma_{p e}^{A k}(h \nu)}{4 \pi}\left[1+\frac{1}{2} \eta_{A k}\left(\frac{3}{2} \sin ^{2} \xi^{\prime}-1\right)\right] \\
& \times\left|E_{2}(z ; h v)\right|^{2}\left[1-R\left(\phi_{p e}^{0}\right)\right] e^{-\frac{z}{\Lambda_{p e} \sin \phi_{p e}^{0}}} .
\end{aligned}
$$

Specifying this now for the polarized case that is found with synchrotron radiation as the source, we have for the total photoemission yield by substituting Eq. (8) into Eq. (10a) and integrating over depth

$$
\begin{aligned}
I_{A k, p e}\left(h v, \hat{\varepsilon}, \phi_{p e}^{0}\right)= & \int_{0}^{\infty} \frac{d z \frac{d I_{A k}\left(z ; h v, \hat{\varepsilon}, \phi_{p e}^{0}\right)}{d z}}{=} \rho_{A} \frac{\sigma_{p e}^{A k}(h \nu)}{4 \pi} A_{A k}\left(\eta_{A k}, \xi\right)\left[1-R\left(\phi_{p e}^{0}\right)\right] \\
& \times \frac{|t|^{2}}{2 \operatorname{Im}\left\{k_{2} \cos \theta_{2}\right\}+\frac{1}{\Lambda_{p e} \sin \phi_{p e}^{0}}},
\end{aligned}
$$

where $A_{A k}\left(\eta_{A k}, \xi\right)=\left[1+\eta_{A k}\left(\frac{3}{2} \cos ^{2} \xi-\frac{1}{2}\right)\right]$.

This is fully specified for computation if we note that $k_{2}=n_{2} \omega / c=2 \pi n_{2} / \lambda_{x}$ and $n_{2}=\alpha+i \beta=1-\delta+i \beta$, and define $n_{2} \cos \theta_{2}=\gamma+i \zeta$.

Then, since $n_{2}^{2} \cos ^{2} \theta_{2}=(\gamma+i \zeta)^{2}=n_{2}^{2}\left(1-\sin ^{2} \theta_{2}\right)$ $=n_{2}^{2}-n_{1}^{2} \sin ^{2} \theta_{1}=(\alpha+i \beta)^{2}-n_{1}^{2} \sin ^{2} \theta_{1}$,

$$
\gamma=\sqrt{\frac{X+\sqrt{X^{2}+Y^{2}}}{2}}, \quad \zeta=\sqrt{\frac{-X+\sqrt{X^{2}+Y^{2}}}{2}},
$$

where $X=\alpha^{2}-\beta^{2}-n_{1}^{2} \sin ^{2} \theta_{1}$ and $Y=2 \alpha \beta$.

In the soft and hard $\mathrm{X}$-ray energy regime, one finds that $\beta \ll 1$ and $\alpha \cong 1$, and we can thus make the following approximation: $\quad \gamma \cong \sqrt{X}$ and $\zeta \cong \frac{Y}{2 \sqrt{X}}=\frac{\alpha \beta}{\sqrt{\alpha^{2}-\beta^{2}-n_{1}^{2} \sin ^{2} \theta_{1}}}$. Since and $k_{2}^{r}=\frac{\omega}{c} \sqrt{\gamma^{2}+n_{1}^{2} \sin ^{2} \theta_{1}}$ and $k_{2}^{r} \cos \theta_{2}=\frac{\omega}{c} \gamma$ $k_{2}^{i}=\frac{\omega}{c} \zeta, \quad n_{1} \sin \theta_{1}=\gamma \tan \theta_{2} . \quad$ Therefore, $\quad n_{1}^{2} \sin ^{2} \theta_{1}$ $=\gamma^{2} \tan ^{2} \theta_{2} \cong\left(\alpha^{2}-\beta^{2}-n_{1}^{2} \sin ^{2} \theta_{1}\right) \tan ^{2} \theta_{2}$, which becomes

$$
n_{1} \sin \theta_{1}=\sqrt{\alpha^{2}-\beta^{2}} \sin \theta_{2}
$$

Finally,

$$
\begin{aligned}
2 \operatorname{Im}\left(k_{2} \cos \theta_{2}\right) & =2 \frac{\omega}{c} \zeta=\frac{4 \pi}{\lambda_{x}} \zeta \\
& =\frac{4 \pi}{\lambda_{x}} \frac{\alpha \beta}{\sqrt{\alpha^{2}-\beta^{2}-n_{1}^{2} \sin ^{2} \theta_{1}}} \\
& \cong \frac{4 \pi}{\lambda_{x}} \frac{\alpha \beta}{\sqrt{\alpha^{2}-\beta^{2}} \cos \theta_{2}} \cong \frac{4 \pi}{\lambda_{x}} \frac{\beta}{\cos \theta_{2}} .
\end{aligned}
$$

When $\sin \theta_{1} \ll \alpha$ (far away from the total external reflection region), we can further make the following approximation:

$$
|t|^{2} \cong \frac{4}{(1+\alpha)^{2}+\beta^{2}} \cong \frac{1}{(1-\delta / 2)^{2}} \cong 1+\delta,
$$

Eq. (11) thus becomes

$$
\begin{aligned}
I_{A k, p e}\left(h v, \hat{\varepsilon}, \phi_{p e}^{0}\right) \approx & \rho_{A} \frac{\sigma_{p e}^{A k}(h \nu)}{4 \pi} A_{A k}(\eta, \xi)\left[1-R\left(\phi_{p e}^{0}\right)\right] \\
& \times \frac{1+\delta}{\frac{4 \pi}{\lambda_{x}} \frac{\beta}{\sin \phi_{2}}+\frac{1}{\Lambda_{p e} \sin \phi_{p e}^{0}}},
\end{aligned}
$$

where $\frac{4 \pi}{\lambda_{x}} \beta=\frac{1}{\Lambda_{x e}}$, with $\Lambda_{x e}=$ the x-ray attenuation length due to absorption in the absence of significant refraction or reflection at the surface, and $\phi_{2}$, as before, is the complement of the angle of incidence $\theta_{2}$ (cf. Fig. 1 and the more general multilayer geometry of Fig. 2). It is notable here that the intensity variation as a function of photon energy appears to be very similar to the shape of the inverse $\beta$ curve around an absorption edge in the soft $\mathrm{x}$-ray range because the second term in the denominator of Eq. (16a) remains almost constant while the numerator $1+\delta$ varies almost indiscernibly. Thus, it is the first term in the denominator, which controls the variation. This result is also consistent with results from $\mathrm{x}$-ray diffraction and scattering theory. ${ }^{49}$ 
As $\theta_{1}$ increases and thus becomes closer to the total external reflection region, $\sin \theta_{1}$ approaches $\alpha$ and Eq. (11) becomes

$$
\begin{aligned}
I_{A k}\left(h v, \hat{\varepsilon}, \phi_{p e}^{0}\right)= & \rho_{A} \frac{\sigma_{p e}^{A k}(h \nu)}{4 \pi} A_{A k}(\eta, \xi)\left[1-R\left(\phi_{p e}^{0}\right)\right] \\
& \times \frac{4}{\left(1+\sqrt{1-\frac{2 \delta}{\phi_{1}^{2}}}\right)^{2}} \frac{1}{\frac{4 \pi}{\lambda_{x}} \frac{\beta}{\sin \phi_{2}}+\frac{1}{\Lambda_{p e} \sin \phi_{p e}^{0}}},
\end{aligned}
$$

where the following approximation can been used:

$$
\begin{aligned}
|t|^{2}= & \frac{4}{\left|n_{2}+\frac{\cos \theta_{2}}{\cos \theta_{1}}\right|^{2}} \cong \frac{4}{\left|n_{2}+\frac{\sqrt{\alpha^{2}-\sin ^{2} \theta_{1}}}{\alpha \cos \theta_{1}}\right|^{2}} \\
& \cong \frac{4}{\left|n_{2}+\frac{\sqrt{\cos ^{2} \theta_{1}-2 \delta}}{\alpha \cos \theta_{1}}\right|^{2}} \\
& \cong \frac{4}{\left|1+\frac{\sqrt{\cos ^{2} \theta_{1}-2 \delta}}{\cos \theta_{1}}\right|^{2}}=\frac{4}{\left|1+\sqrt{1-\frac{2 \delta}{\sin ^{2} \phi_{1}}}\right|^{2}} \\
& \approx \frac{4}{\left|1+\sqrt{1-\frac{2 \delta}{\phi_{1}^{2}}}\right|^{2}},
\end{aligned}
$$

with additional simplifications of $\sqrt{\alpha^{2}-\sin ^{2} \theta_{1}}=$ $\sqrt{(1-\delta)^{2}-\sin ^{2} \theta_{1}} \cong \sqrt{\cos ^{2} \theta_{1}-2 \delta} \quad$ and $\quad 1 \gg \phi_{1}^{2}>2 \delta$. Near an absorption edge in the soft $\mathrm{x}$-ray range, the factor $\frac{4}{\left(1+\sqrt{1-\frac{2 \delta}{\phi_{1}^{2}}}\right)^{2}}$ plays a more critical role than the factor $\frac{1}{\frac{4 \pi}{\beta} \sin \phi_{2}}+\frac{1}{\Lambda_{p e} \sin \phi_{p e}^{0}}$ in intensity variation as a function of photon energy when $2 \delta$ is comparable to $\phi_{1}^{2}$, corresponding to the critical angle.

Thus, $\delta$ determines the intensity shape. In the total external reflection region $\left(\phi_{1}^{2}<2 \delta\right) \xi \cong \sqrt{\sin ^{2} \theta_{1} / \alpha^{2}-1},|t|^{2} \cong$ $\frac{2 \phi_{1}^{2}}{\delta}$ and $\operatorname{Im}\left\{k_{2} \cos \theta_{2}\right\} \cong \frac{2 \pi}{\lambda} \sqrt{2 \delta-\phi_{1}^{2}}$. Therefore, in the region of total external reflection,

$$
\begin{aligned}
I_{A K}\left(h v, \hat{\varepsilon}, \theta_{p e}^{0}\right) & =\rho_{A} \frac{\sigma_{p e}^{A K}(h v)}{4 \pi} A_{A K}\left(\eta_{A K}, \xi\right) \\
& \times\left[1-R\left(\theta_{p e}^{0}\right)\right] \frac{\frac{2 \phi_{1}^{2}}{\delta}}{\frac{4 \pi}{\lambda_{x}} \sqrt{2 \delta-\phi_{1}^{2}}+\frac{1}{\Lambda_{p e} \sin \theta_{p e}^{0}}} .
\end{aligned}
$$

We note that the variation of the photoemission intensity is predominantly determined by $\delta$ in the total external reflection region since the $\mathrm{x}$-ray penetration depth depends more on $\delta$ rather than $\beta$, a result that is consistent with well-known limits in x-ray diffraction and scattering theory. ${ }^{49}$ Below, we illustrate the applicability of these limiting approximations in comparison to experimental data for $\mathrm{MnO}$.
It is convenient to express spectral intensities in terms of reflectance $R$. Reflectance $R$ and transmittance $T$ are defined with respect to the Poynting vector $\mathbf{S}$ and the interface normal $\mathbf{n}$;

$$
R=\frac{\left|\mathbf{n} \cdot \mathbf{S}_{\mathbf{1}}^{\prime}\right|}{\left|\mathbf{n} \cdot \mathbf{S}_{\mathbf{1}}\right|}, \quad T=\frac{\left|\mathbf{n} \cdot \mathbf{S}_{2}\right|}{\left|\mathbf{n} \cdot \mathbf{S}_{\mathbf{1}}\right|} .
$$

These can be simply reduced to the following using Eqs. (3):

$$
R=|r|^{2}, \quad T=\frac{\left|n_{2} \cos \theta_{2}\right|}{\left|n_{1} \cos \theta_{1}\right|}|t|^{2} .
$$

The energy flow is attenuated via the imaginary channel $\operatorname{Im}\left\{k_{2} \cos \theta_{2}\right\}$, thus resulting in no energy flow along the $\mathrm{z}$-direction for the semi-infinite case. Therefore, since the energy is transferred along the $\mathbf{z}$-axis (or $\mathbf{n}$-direction) via the real channel $\operatorname{Re}\left\{k_{2} \cos \theta_{2}\right\}$ only. Equation (18) should be modified for our conducting medium as follows:

$$
R=|r|^{2}, T=\frac{\operatorname{Re}\left\{n_{2} \cos \theta_{2}\right\}}{\left|n_{1} \cos \theta_{1}\right|}|t|^{2} .
$$

It can also be readily shown that $R+T=1$ in the presence of absorption, and Eq. (11) can be rewritten as a function of $R$ as follows:

$$
\begin{aligned}
& I_{A k}\left(h v, \hat{\varepsilon}, \phi_{p e}^{0}\right)=\rho_{A} \frac{\sigma_{p e}^{A k}(h \nu)}{4 \pi} A_{A k}\left(\eta_{A k}, \xi\right)\left[1-R\left(\phi_{p e}^{0}\right)\right] \\
& \times \frac{(1-R) \frac{\cos \theta_{1}}{\operatorname{Re}\left\{n_{2} \cos \theta_{2}\right\}}}{\frac{4 \pi}{\lambda_{x}} \operatorname{Im}\left\{k_{2} \cos \theta_{2}\right\}+\frac{1}{\Lambda_{p e} \sin \phi_{p e}^{0}}} \\
& =\rho_{A} \frac{\sigma_{p e}^{A k}(h \nu)}{4 \pi} A_{A k}\left(\eta_{A k}, \xi\right)\left[1-R\left(\phi_{p e}^{0}\right)\right] \\
& \times \frac{(1-R) \frac{\cos \theta_{1}}{\cos \theta_{2}}}{\frac{4 \pi}{\lambda_{x}} \frac{\beta}{\sin \phi_{2}}+\frac{1}{\Lambda_{p e} \sin \phi_{p e}^{0}}} .
\end{aligned}
$$

Finally, we consider $\mathrm{x}$-ray emission from a semi-infinite sample, and the x-ray optical effects that influence its intensities. The electric fields of the outgoing wave (emitted photon) inside and outside a sample can be written as, respectively,

$$
\begin{aligned}
& \mathbf{E}_{2 x e}\left(\mathbf{r}, t ; h \nu_{x e}\right)=\mathbf{E}_{2 x e}\left(z=0^{-}\right) e^{i\left(\mathbf{k}_{2 x e} \cdot \mathbf{r}-\omega t\right)}, \\
& \mathbf{E}_{2 x e}^{\prime}\left(\mathbf{r}, t ; h \nu_{e m}\right)=\mathbf{E}_{2 x e}^{\prime}\left(z=0^{-}\right) e^{i\left(\mathbf{k}_{2 x e}^{\prime} \cdot \mathbf{r}-\omega t\right)}, \\
& \mathbf{E}_{1 x e}\left(\mathbf{r}, t ; h \nu_{e m}\right)=\mathbf{E}_{1 x e}\left(z=0^{+}\right) e^{i\left(\mathbf{k}_{1 e e} \cdot \mathbf{r}-\omega t\right)},
\end{aligned}
$$

and according to Snell's law $n_{2} \cos \phi_{2 x e}=n_{1} \cos \phi_{1 x e}^{0}$, the complex angle $\phi_{2 x e}$ inside the sample (medium 2) can be calculated from the actual measurement at the take-off angle $\phi_{1 x e}^{0}$ at which the external x-ray detector counts the number of emitted photons within a very narrow solid angle. The Fresnel coefficients are

$$
\begin{aligned}
r_{x e}^{s} & =\frac{n_{2} \sin \phi_{2 x e}-n_{1} \sin \phi_{1 x e}^{0}}{n_{1} \sin \phi_{1 x e}^{0}+n_{2} \sin \phi_{2 x e}}, \\
t_{x e}^{s} & =\frac{2 n_{2} \sin \phi_{2 x e}}{n_{1} \sin \phi_{1 x e}^{0}+n_{2} \sin \phi_{2 x e}} \text { (s-polarization), }
\end{aligned}
$$




$$
\begin{aligned}
r_{x e}^{p} & =\frac{n_{2} \sin \phi_{1 x e}^{0}-n_{1} \sin \phi_{2 x e}}{n_{2} \sin \phi_{1 x e}^{0}+n_{1} \sin \phi_{2 x e}}, \\
t_{x e}^{p} & \left.=\frac{2 n_{2} \sin \phi_{2 x e}}{n_{2} \sin \phi_{1 x e}^{0}+n_{1} \sin \phi_{2 x e}} \text { (s-polarization }\right),
\end{aligned}
$$

where $E_{2 x e}^{\prime}\left(z=0^{+}\right)=r_{x e} E_{2 x e}\left(z=0^{+}\right)$and $E_{1 x e}\left(z=0^{-}\right)$ $=t_{x e} E_{2 x e}\left(z=0^{+}\right)$.

Therefore, the electric field strength of the emitted $\mathrm{X}$-rays outside the sample, at a position given by $\mathbf{r}_{\text {probe }}$, is given by

$$
\begin{aligned}
E_{1 x e}\left(\mathbf{r}_{\text {probe }}, z ; h \nu_{x e}, \theta_{1 x e}\right) & =E_{1 x e}\left(z=0^{-}\right) e^{i\left(\mathbf{k}_{1 x e} \cdot \mathbf{r}_{\text {probe }}-\omega t\right)} \\
& =t_{x e} E_{2 x e}\left(\mathbf{r} ; h \nu_{x e}\right) e^{i\left(\mathbf{k}_{1 x e} \cdot \mathbf{r}_{\text {probe }}-\mathbf{k}_{2 x e} \cdot \mathbf{r}\right)}
\end{aligned}
$$

The electric field $E_{2 x e}\left(\mathbf{r} ; h \nu_{x e}\right)$ represents the intensity of emitted photons. Indeed the cross section, $\frac{d \sigma_{x e}(h \nu, \hat{\varepsilon})}{d \Omega}$ represents $E_{2 x e}\left(\mathbf{r} ; h \nu_{x e}\right)$, and we will for convenience let $\left|E_{2 x e}\left(\mathbf{r} ; h \nu_{x e}\right)\right|=1$ later on. Since $\mathbf{k}_{1 x e} \cdot \mathbf{r}_{\text {probe }}$ is a real number, the squared electric field probed with a detector in vacuum becomes

$$
\left|E_{1 x e}\left(\mathbf{r}_{\text {probe }}, z ; h \nu_{x e}, \theta_{1 x e}\right)\right|^{2}=\left|t_{x e}\right|^{2} e^{-2 \operatorname{Im}\left\{k_{2 x e} z \sin \phi_{2 x e}\right\}} .
$$

The integrated total $\mathrm{x}$-ray emission yield for $k$-hole decay in an $A$-atom can now be written as

$$
\begin{aligned}
& I_{A k, x e}\left(\mathbf{r}_{\text {probe }} ; h \nu, \hat{\varepsilon}, \phi_{1 x e}^{0}\right) \\
& =\int_{0}^{\infty} d z \frac{d I_{A k, x e}\left(\mathbf{r}_{\text {probe }} ; h \nu, \hat{\varepsilon}, \phi_{1 x e}^{0}\right)}{d z} \\
& =\rho_{A} \frac{d \sigma_{x e}(h \nu, \hat{\varepsilon})}{d \Omega} \int_{0}^{\infty} d z|t|^{2}\left|t_{x e}\right|^{2} e^{-2 \operatorname{Im}\left\{k_{2} z \sin \phi_{2}\right\}-2 \operatorname{Im}\left\{k_{2 x e} z \sin \phi_{x e}^{0}\right\}} \\
& =\rho_{A} \frac{d \sigma_{x e}^{A k}(h \nu)}{d \Omega} \frac{|t|^{2}\left|t_{x e}\right|^{2}}{2 \operatorname{Im}\left\{k_{2} \sin \theta_{2 x}+k_{2 x e} \sin \phi_{2 x e}\right\}}
\end{aligned}
$$

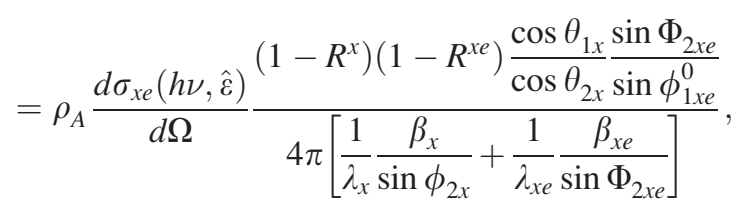

where $\gamma_{x e}$ and $\zeta_{x e}$ are defined from $n_{2} \cos \phi_{2 x e}=\gamma_{x e}+i \zeta_{x e}$.

\section{B. A multilayer sample}

Figure 2 shows the schematic configuration of a generic multilayer with interdiffusion between its constituent layers. In general, as discussed previously by Windt ${ }^{50}$ and following a method developed earlier by Parratt, ${ }^{42}$ the electric field in the $i$ th individual layer that is adjacent to a $j$ th individual layer can be written as

$$
\begin{array}{r}
E_{i}\left(z_{i}\right)=\frac{1}{t_{i j}} e^{-i \chi_{i}\left(z_{i}\right)} E_{j}(0)+\frac{r_{i j}}{t_{i j}} e^{-i \chi_{i}\left(z_{i}\right)} E_{j}^{\prime}(0), \\
E_{i}^{\prime}\left(z_{i}\right)=\frac{r_{i j}}{t_{i j}} e^{i \chi_{i}\left(z_{i}\right)} E_{j}(0)+\frac{1}{t_{i j}} e^{i \chi_{i}\left(z_{i}\right)} E_{j}^{\prime}(0),
\end{array}
$$

where $E_{i}\left(z_{i}\right)$ and $E_{i}^{\prime}\left(z_{i}\right)$ are incoming (increasing z) and outgoing (decreasing $\mathrm{z}$ ) fields at a distance $z_{i}$ above the interface between $i$ and $j$ layers, respectively, and $\chi_{i}\left(z_{i}\right)=$ $2 \pi z_{i} n_{i} \cos \theta_{i} / \lambda$. Also, $E_{j}^{+}(0)$ and $E_{j}^{-}(0)$ are the field amplitudes at the top of the $j$ th layer, and the Fresnel coefficients in case of p-polarization are given by

$$
\begin{aligned}
r_{i j}^{P} & =\frac{\left|\mathbf{E}_{i}^{\prime}\right|}{\left|\mathbf{E}_{i}\right|}=\frac{n_{i} \cos \theta_{j}-n_{j} \cos \theta_{i}}{n_{i} \cos \theta_{j}+n_{j} \cos \theta_{i}}, \\
t_{i j}^{P} & =\frac{\left|\mathbf{E}_{j}\right|}{\left|\mathbf{E}_{i}\right|}=\frac{2 n_{i} \cos \theta_{i}}{n_{i} \cos \theta_{j}+n_{j} \cos \theta_{i}} .
\end{aligned}
$$

The net reflection $\left(r_{i}\right)$ and transmission $\left(t_{i}\right)$ of the $i$ th layer are given by

$$
r_{i}=\frac{r_{i j}+r_{j} e^{2 i \chi_{i}}}{1+r_{i j} r_{j} e^{2 i \chi_{i}}}, \quad t_{i}=\frac{t_{i j} t_{j} e^{2 i \chi_{i}}}{1+r_{i j} r_{j} e^{2 i \chi_{i}}}
$$

The total reflectance $R$ and transmittance $T$, which measure the fraction of energy that is reflected at and transmitted through the entire multilayer sample, are then

$$
R=|r|^{2}, \quad T=\operatorname{Re}\left\{\frac{n_{s} \cos \theta_{s}}{n_{v} \cos \theta_{v}}\right\}|t|^{2},
$$

where subscripts $s$ and $v$ stand for substrate and vacuum, respectively. Absorptance A, which measures the amount of energy absorbed by the multilayer sample, is defined as $A=1-R-T$. We use a recursive algorithm to compute the field amplitudes throughout the stack, starting at the bottommost layer $(i=N, j=s$ ) with the field amplitudes in the substrate given by $\left|\mathbf{E}_{s}(0)\right|=1$ and $\left|\mathbf{E}_{s}^{\prime}(0)\right|=0$. The net total reflection and transmission coefficients of the film can then be computed from the field amplitudes in vacuum

$$
r=\frac{E_{v}^{\prime}(0)}{E_{v}(0)}, \quad t=\frac{1}{E_{v}(0)} .
$$

Once the transmission coefficient is computed from Eq. (28), the field amplitudes can be rescaled using $E(z) \rightarrow t E(z)$. The squared electric field strength in the $i$ th layer is finally obtained from

$$
I_{i}(z)=\left|E_{i}(z)+E_{i}{ }^{\prime}(z)\right|^{2}=\left[\begin{array}{l}
\left|E_{i}(z)+E_{i}{ }^{\prime}(z)\right|^{2}(\text { s-polarization }) \\
\left|\left[E_{i}(z)-E_{i}{ }^{\prime}(z)\right] \cos \theta_{i}\right|^{2}+\left|\left[E_{i}(z)+E_{i}{ }^{\prime}(z)\right] \sin \theta_{i}\right|^{2}(\text { p-polarization })
\end{array}\right] .
$$


To deal with more realistic multilayers, we also need to take into account interfacial roughness and/or interdiffusion by replacing abrupt interfaces by gradually varying layers with thickness $2 \sigma_{i}$. We model such non-ideal interfaces in our calculations using linear, elliptical, half-Gaussian, and half-Lorentzian functions. This is done by breaking the interface into several interfaces, and calculating refraction and reflection at each interface. The reduction of reflection and refraction caused by the interdiffusion at the interfaces are also taken into account. ${ }^{29}$

Proceeding by complete analogy from the semi-infinite layer, the differential photoemission intensity originating from $k$-atom orbital of $A$-atom at depth $\mathrm{z}$ in the $i$ th layer is given by

$$
\begin{aligned}
\frac{d I_{A k, p e}^{i}(z ; h \nu, \hat{e})}{d z}= & \rho_{A}^{i} \frac{\sigma_{p e}^{A k}(h \nu)}{4 \pi} A\left(\eta, \xi^{i}\right) I_{i}(z ; h \nu, \hat{e})\left[1-R\left(\phi_{p e}^{i}\right)\right] \\
& \times \exp \left(-\frac{z-\sum_{l=1}^{i-1} d_{l}}{\Lambda_{p e}^{i A k} \sin \phi_{p e}^{i}}\right) \\
& \times \prod_{q=1}^{i-1} \exp \left(-\frac{d_{q}}{\Lambda_{p e}^{q A k} \sin \phi_{p e}^{q}}\right)
\end{aligned}
$$

where the multiplication of exponential factors in the above equation implies that a photoelectron created in the $i$ th layer experiences different attenuation in each layer until it reaches the surface. Finally, we obtain the total spectral intensity from the $k$ subshell of atom A

$$
\begin{aligned}
I_{A k, p e}(h \nu)= & \sum_{i \in F(A k)} \int_{\Sigma_{k=1}^{i-1} d_{k}}^{\Sigma_{l=1}^{i} d_{l}} d z \frac{d I_{A k}^{i}(z ; h \nu, \hat{e})}{d z} \\
= & \frac{\sigma_{p e}^{A k}(h \nu)}{4 \pi} \sum_{i \in F(A k)} \rho_{A}^{i} A\left(\eta, \xi^{i}\right) \\
& \times \prod_{q=1}^{i-1} \exp \left(-\frac{d_{q}}{\Lambda_{p e}^{q A k} \sin \phi_{p e}^{q}}\right) \\
& \times \int_{\sum_{k=1}^{i-1} d_{k}}^{\Sigma_{l=1}^{i} d_{l}} d z I_{i}(z ; h \nu, \hat{e})\left[1-R\left(\phi_{p e}^{i}\right)\right] \\
& \times \exp \left(-\frac{z-\sum_{l=1}^{i-1} d_{l}}{\Lambda_{p e}^{i A k} \sin \phi_{p e}^{i}}\right),
\end{aligned}
$$

where $F(A k)$ signifies the set of layers in the multilayer that contains $A$ atoms, which can emit electrons from subshell $k$.

Similar to photoelectron emission, the differential intensity for $\mathrm{x}$-ray emission due to a $k$-subshell decay in atom $A$ from depth $z$ will be given by $\frac{d I_{A k, x e}^{i}(z ; h \nu, \hat{e})}{d z}=\rho_{A}^{i} \frac{d \sigma_{x e}^{A k}(h \nu, \hat{e})}{d \Omega} I_{i}(z ; h \nu, \hat{e}) I_{i}^{x e}\left(z ; h \nu_{x e}, \hat{e}, \phi_{x e}^{i}\right)$,

where $I_{i}^{x e}\left(z ; h \nu_{x e}, \phi_{x e}^{i}\right)=\left|E_{\text {vacuum }}^{x e-}\left(z ; h \nu_{x e}, \phi_{x e}^{i}\right)\right|^{2}$ can be calculated by taking into account that the emitted x-ray in the $i$ th layer is illuminated from below. Since the emitted photons undergo self-absorption in each layer and reflection/refraction at each interface, the calculation becomes more complicated than photoelectron emission.

Therefore, the total yield of XES can be obtained as follows:

$$
\begin{aligned}
I_{x e}(h \nu)= & \sum_{i \in F(A k)} \int_{\Sigma_{k=1}^{i-1} d_{k}}^{\sum_{l=1}^{i} d_{l}} d z \frac{d I_{A k, x e}^{i}(z ; h \nu)}{d z} \\
= & \frac{d \sigma_{x e}^{A k}(h \nu)}{d \Omega} \sum_{i \in F(A k)} \rho_{A}^{i} \int_{\sum_{k=1}^{i-1} d_{k}}^{\Sigma_{l=1}^{i} d_{l}} d z I_{i}(z ; h \nu, \hat{e}) \\
& \times I_{i}^{x e}\left(z ; h \nu_{e m}, \hat{e}, \phi_{x e}^{i}\right) .
\end{aligned}
$$

\section{Standing-wave effects}

Finally, we comment on the way in which standingwave effects will affect various emission intensities. Fig. 1 illustrates the basic interference, which results in any situation in which the specular reflectivity $R$ is $>0$. A standing wave with wavefronts parallel to the reflecting surface is created, and simple geometric considerations indicate that the period of the square of the electric field that is responsible for exciting electron or $\mathrm{x}$-ray emission will be given by

$$
\lambda_{S W}\left(\left|E^{2}\right|\right)=\lambda_{x} / 2 \sin \phi_{i n c} .
$$

As indicated in Fig. 2, electron or x-ray emission from a given point in the standing wave will thus be modulated in depth, as discussed more quantitatively below.

It is also important to note that what is of interest here is the total wave field intensity inside a sample that may consist of multiple layers, or layers grown on a suitable multilayer mirror, although we have here simplified the argument to consider the region in vacuum above a reflecting surface or multilayer. Thus, the detailed $\mathrm{x}$-ray optical effects inside the sample, however defined, must in general be modeled, as discussed in the preceding paragraphs and further below. All of the calculations shown here will include all of these effects via the YXRO program. If such standing waves are produced by Bragg reflection from crystal planes or from a multilayer mirror, then the condition for strong reflection is

$$
p \lambda_{x}=2 d \sin \phi_{B}
$$

where $p=1,2,3, \ldots$ and $d$ is the spacing of the planes or the multilayer period. This implies via Eq. (34) that the period of the standing wave created equals the interplanar spacing or the multilayer period, although refraction and reflection in the multilayer may cause slight variations from this form inside a multilayer sample. Varying either the angle around 
the Bragg condition for a given photon energy or the photon energy around the Bragg condition for a given angle changes the phase of the standing wave by about $\pi$, perpendicular to the reflecting planes. We will illustrate the utility of these properties of standing waves for depth-resolved emission experiments below.

In addition, we note that additional reflections in a given multilayer structure of total thickness $D$ can give rise to further standing-wave effects that are often termed Kiessig fringes ${ }^{29}$ or Fresnel fringes, with a similar equation describing the maxima in reflectivity associated with these fringes

$$
q \lambda_{x}=2 D \sin \phi_{K}
$$

where $q$ is the (usually unknown) order of the diffraction and $\phi_{K}$ is the incidence angle at which a maximum occurs. A useful approximate relationship for data interpretation is to note that, if the angle $\phi_{K}$ is less than about $20^{\circ}, \sin \phi_{K}$ can be replaced by $\phi_{K}$, and a simple derivation then shows that the angular spacing between two adjacent fringes $\Delta \phi_{K}$ is given by

$$
\Delta \phi_{K}\left(^{\circ}\right)=\frac{\lambda_{x}}{2 D}=\frac{3.552 \times 10^{5}}{h \nu(e V) D(\AA)},
$$

which can be used to check experimental data for selfconsistency with the often-known structure of the multilayer.

Finally, we note that in case of either Bragg reflection or the Kiessig fringes, the fact that the standing-wave modulation of $|\vec{E}|^{2}$ that is responsible for electron or x-ray emission is the result of interference between the incident and reflected fields, implies that its intensity will (in vacuum) range over maximum limits set by $1+R \pm 2 \sqrt{R}$. Thus, even a modest reflectivity at the Bragg condition of 0.01 will yield an overall SW modulation of $20 \%$ via the last term in this expression. In total reflection, the modulation of the SW will go from 0 to 4 times the incident intensity, or a total change of 4 times the incident intensity. This relationship is more complex inside of a multilayer sample, but nonetheless is semi-quantitatively valid as a predictor of the fractional effects to be seen in SW spectroscopies.

\section{RESULTS FOR EXAMPLE SYSTEMS AND DISCUSSION}

All of the above formalism has been incorporated into the program YXRO, which has been tested in numerous studies against both programs predicting multilayer reflectivities $^{50,51}$ as well as experimental data from several standingwave experimental studies. ${ }^{18,20-32}$

\section{A. Semi-infinite sample, non-resonant excitation}

As a first case, we consider the simple case of photoelectron emission from a semi-infinite substrate with nonresonant excitation, and focus on what occurs as the incidence angle $\phi_{1}$ in Fig. 1 goes below the critical angle and into the total reflection condition. The optical constants can in this case be taken directly from a standard tabulation of the Berkeley Center for X-Ray Optics, ${ }^{51}$ and the electron inelastic mean free path from semi-empirical calculations based on optical constants. ${ }^{48}$ We choose here to perform the same calculation as Henke ${ }^{9}$ for $\mathrm{Au} 4 f_{7 / 2}$ excited from Au by Al $K \alpha$ radiation to yield a kinetic energy of $1402 \mathrm{eV}$ and to compare our results to his experimental data. A comparison of calculations based on the YXRO program and Henke's experimental data is shown in Fig. 3 for two choices of dielectric constants at this photon energy: those from the standard tabulation $\left(\delta=1.00 \times 10^{-3}\right.$ and $\left.\beta=2.89 \times 10^{-4}\right)$, and values derived by Henke by empirically fitting the behavior of the $\mathrm{Au} 4 \mathrm{f}_{7 / 2}$ intensity with angle $(\delta=6.70$ $\times 10^{-4}$ and $\left.\beta=2.30 \times 10^{-4}\right)$. Both calculations agree well with the experimental data, although the empirically adjusted dielectric constants of course fit better. The undershot of theory at low angles may have to do with the angular spread in the $\mathrm{x}$-ray source, which was about $0.45^{\circ}$.

This kind of enhancement of photoelectron intensity for incidence angles below the critical angle is thus expected to be a general phenomenon. Another important aspect of this comparison to experiment is the essentially constant nature of the intensity for larger angles of incidence, which is directly linked to the fact that the x-ray source in this case illuminates a wide area of the sample as seen by the electron spectrometer, a typical situation with a laboratory x-ray source. If, on the other hand, the beam is focused such that, for approximately normal electron emission, it does not fill the area of the sample seen by the spectrometer (a typical situation in synchrotron radiation experiments), then the intensity falls rapidly as the incidence angle is increased above the critical angle, due to a simple geometric effect. ${ }^{52}$ The YXRO program permits calculations in both situations as user-selected options.

\section{B. Semi-infinite sample, resonant excitation}

We now consider a more complex case of a semiinfinite sample for which the photon energy is scanned through a strong core-level absorption resonance. $\mathrm{MnO}$ has

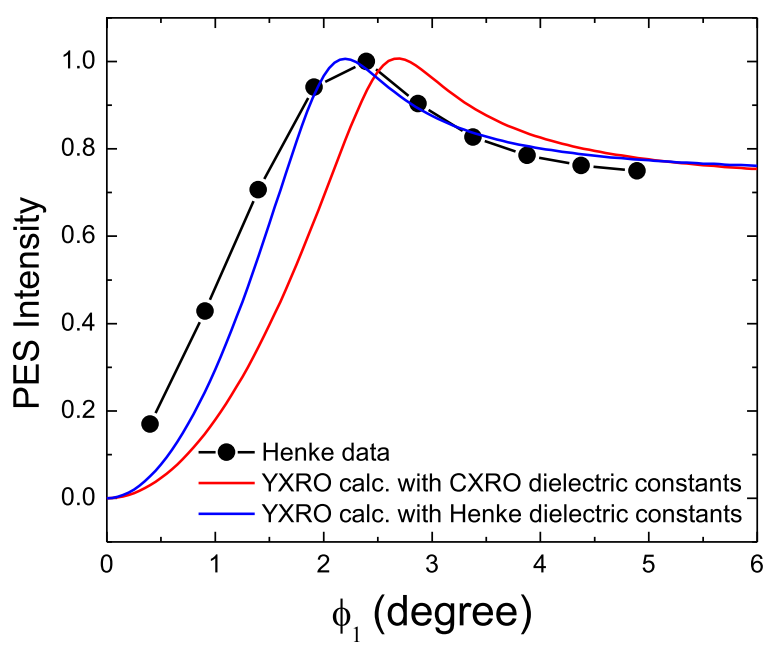

FIG. 3. Experimental data for Au $4 f$ emission from Au at a photon energy of $1486.7 \mathrm{eV}$, as a function of incidence angle on going into total reflection from Ref. 9, are compared to calculations using the methodology and YXRO program described here. Two different sets of optical constants have been used: those from the CXRO database ${ }^{51}$ and those determined in Ref. 9 so as to fit experiment. 
been chosen to simulate $\mathrm{x}$-ray optical effects in both photoemission and $\mathrm{x}$-ray emission spectra for this case, because Mn has strong $2 p_{3 / 2}$ and $2 p_{1 / 2}\left(\mathrm{~L}_{2,3}\right)$ absorption edges in the soft $\mathrm{x}$-ray region at $\sim 640 \mathrm{eV}$. Moreover, the binding energy of the $\mathrm{O} 1 s$ orbital $(\sim 532 \mathrm{eV})$ is significantly lower than the Mn $2 p$ edges, thus making it easy for us to investigate the variation of $\mathrm{O} 1 s$ as a function of incidence angle and photon energy near the Mn $2 p$ edges. This system has been studied in detail previously with reference to what has been termed multi-atom resonant photoemission (MARPE) ${ }^{53}$ Fig. 4(a) shows the optical constants of $\mathrm{MnO}$ around $\mathrm{Mn} L_{2,3}$ edges, which have been obtained from a Mn $L$ edge absorption spectrum that yields $\beta$ and then using Kramers-Kronig analysis to derive $\delta$. The details of obtaining the optical constants from the absorption spectra are described elsewhere. ${ }^{54}$ As mentioned in the previous chapter, when $\delta>0$, we have a finite positive critical angle and a total external reflection region exists for non-zero incidence angles, whereas when $\delta<0$, there is no such total external reflection region in the limits of $|\delta|, \beta \ll 1$. Therefore, total reflection for non-zero incidence is impossible at photon energies below $h \nu \sim 636 \mathrm{eV}$ and around $646 \mathrm{eV}$ from (Fig. 4(a)), which can also be confirmed from the reflectivity calculation shown in Fig. 4(b): in particular, the energy ranges that have finite critical angles, i.e., total reflection regions, exhibit enhanced reflectivity when $\phi_{1}<5^{\circ}$ in contrast with the other energy ranges.
Figs. 4(c) and 4(d) plot x-ray attenuation lengths $\left(\Lambda_{\mathrm{x}}\right)$ and penetration depths inside $\mathrm{MnO}$ at the photon energy corresponding to the $\mathrm{Mn} L_{3}$ edge resonance $(h \nu=636.4 \mathrm{eV})$ and off-resonance (at $h \nu=611 \mathrm{eV}$ ) that are calculated from Eqs. (8) and (15). X-ray attenuation lengths are seen to strongly depend on photon energy near the resonance region; $\Lambda_{\mathrm{x}}$ is $\sim 3300 \AA$ off resonance and only $127 \AA$ on resonance when $\phi_{1}$ is close to $90^{\circ}$ whereas $\Lambda_{\mathrm{x}}$ is $\sim 130 \AA$ off resonance and only $15 \AA$ on resonance when $\phi_{1}$ is near $0^{\circ}$. In addition, $\Lambda_{\mathrm{x}}$ off-resonance is larger than $\Lambda_{\mathrm{x}}$ on-resonance by an order of magnitude or more for all $\phi_{1}$ values.

Figs. 5(a) and 5(c) show the electric field strengths $\left(|\mathrm{E}|^{2}\right)$ and $\mathrm{O} 1 \mathrm{~s}$ photoelectron intensity as a function of depth and incidence angle for $h \nu=611 \mathrm{eV}$ off-resonance, with similar plots for $h \nu=636.4 \mathrm{eV}$ on-resonance in Figs. 5(b) and 5(d). On-resonance, the reflectivity becomes more significant at low incidence angles $\left(\phi_{1}<10^{\circ}\right)$ than it does for offresonance, thus resulting in enhanced standing-wave modulation in the electric field above the surface $\left|E_{\text {vacuum }}(\mathrm{z})\right|^{2}$. A cut through these contours at constant incidence angle would reveal a SW whose period is given by Eq. (34), and the variation of the period with incidence angle is clear in Figs. 5(a) and 5(b). The approach of $|E|^{2}$ above the surface to its maximum value of 4 at total reflection for the on-resonance results is also seen in Figs. 5(a) and 5(b). In contrast, the electric field below the surface, $\left|E_{\mathrm{MnO}}(\mathrm{z})\right|^{2}$, as the resonance is approached is much smaller compared to that off the
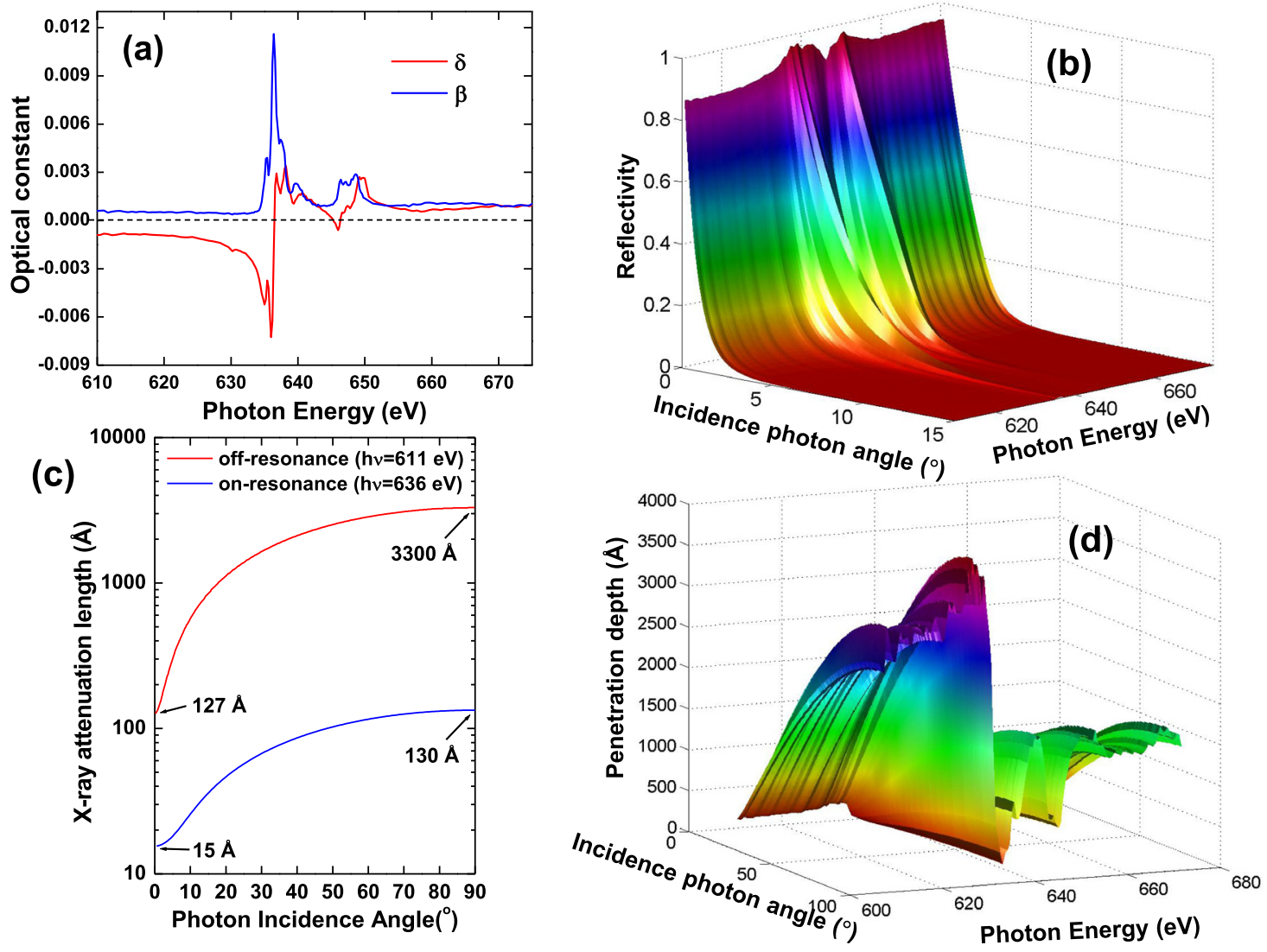

FIG. 4. X-ray optical simulations related to the O $1 s$ photoelectron intensity from $\mathrm{MnO}$ as photon energy scans through the Mn $2 p$ edges: (a) optical constants in the index of refraction $n=1-\delta+i \beta$ across the Mn $2 p$ edge, (b) a 3D representation of calculated reflectivity vs. incidence photon angle and energy, (c) calculated x-ray penetration depth vs. photon incidence angle: red solid curve (off resonance, $h \nu=611 \mathrm{eV}$ ) and blue solid curve $($ on resonance, $h \nu=636.4 \mathrm{eV}$ ), and (d) a 3D representation of the x-ray penetration vs. incidence photon angle and energy. 


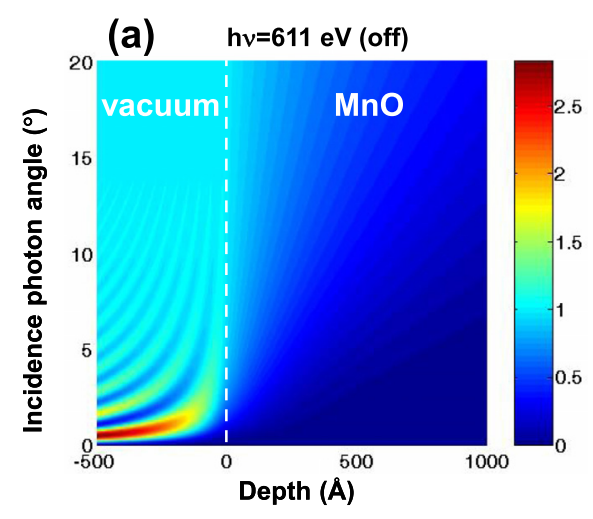

(c) $\mathrm{hv}=611 \mathrm{eV}$ (off)
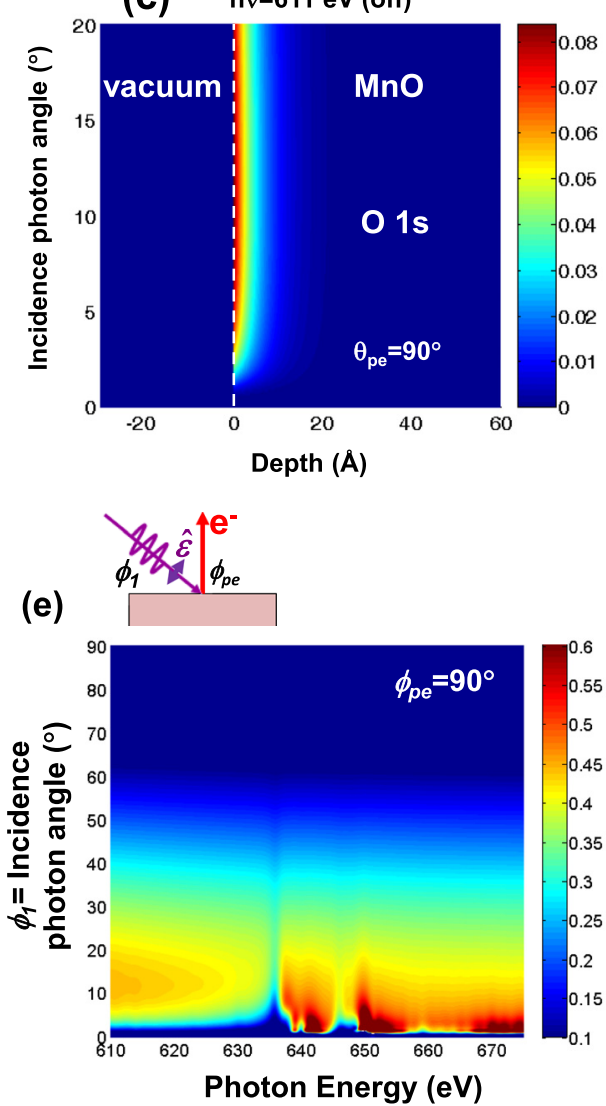

(b) $h v=636.4 \mathrm{eV}$ (on)

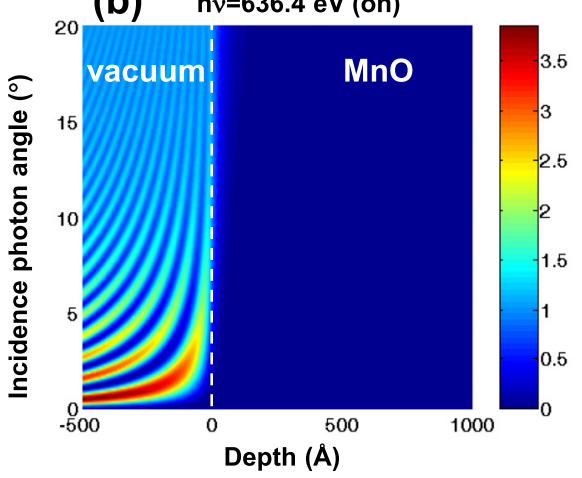

(d)

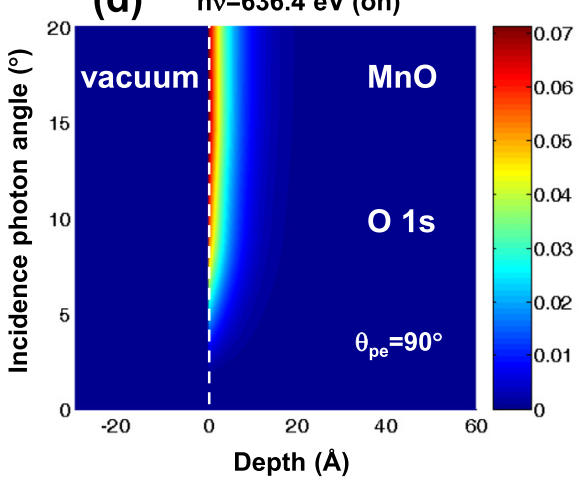

(f)

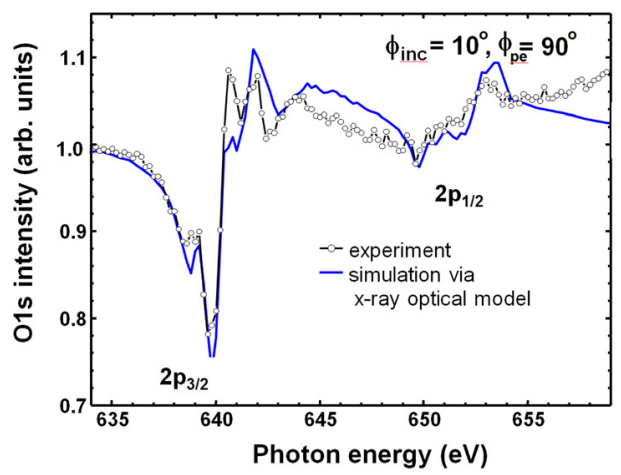

FIG. 5. Calculated p-polarized x-ray electric field strength $|E|^{2}$ ((a) and (b)) and depth-resolved $\mathrm{O} 1 \mathrm{~s}$ photoelectron intensity profiles ((c) and (d)) as a function of depth for $\mathrm{MnO}$ for energies on and off the Mn $2 p_{3 / 2}$ edge as a function of incidence photon angle: (a) and (c) off-resonance, (b) and (d) on-resonance, (e) a 3D representation of $\mathrm{O} 1 \mathrm{~s}$ intensity vs. incidence photon angle and energy at a $90^{\circ}$ take-off angle, and (f) a direct comparison of experiment and theory for O $1 \mathrm{~s}$ emission from $\mathrm{MnO}$ based on data from Ref. 54. resonance. It is important to realize that the photoemission intensity strongly depends on both the electron inelastic mean-free path and the x-ray penetration depth for regions close to total reflection. With increasing $\phi_{1}$, the reflectivity is negligibly small (very little modulation of $\left|E_{\text {vacuum }}(\mathrm{z})\right|^{2}$ and $\beta$ begins to affect the x-ray penetration depth). However, $\left|E_{\mathrm{MnO}}(\mathrm{z}=0)\right|^{2}$ makes a great difference in the behavior of photoemission intensity for small $\phi_{1}\left(<20 \sim 30^{\circ}\right)$, where $\delta$ plays a key role, by contrast with the behavior for relatively larger $\phi_{1}\left(>30^{\circ}\right)$, where the $\mathrm{x}$-ray penetration depth, via $\beta$ is important.

Fig. 5(e) finally shows the PES intensity variation of $\mathrm{O}$ $1 s$ as functions of $h \mathrm{v}$ and $\phi_{1}$. A normal take-off angle $\left(\phi_{\mathrm{pe}}=90^{\circ}\right)$ and $\mathrm{p}$-polarized incident radiation have been used for the calculations, as shown in the inset. For this geometry, normal photon incidence angle $\left(\phi_{1}=90^{\circ}\right)$ must yield zero photoemission intensity from the $\mathrm{O} 1 \mathrm{~s}$ core level, due to the fact that its asymmetry parameter is 2.0 (see, $A(\eta, \xi)$ in Eq. (11)). Maximum O $1 s$ PES intensity occurs at $\phi_{1} \sim 12^{\circ}$ since $A(\eta, \xi)$ decreases with increasing $\phi_{1}$, while $\mathrm{x}$ ray attenuation becomes weaker. The form of these curves for a given incidence angle has been verified for both $\mathrm{MnO}^{54}$ as well as $\mathrm{NiO},{ }^{14}$ in prior studies, where $\mathrm{x}$-ray optical calculations are compared directly to experimental data.

In the region far away from the total reflection $\left(\phi_{1} \gg\right.$ the critical angle), the shape of the $\mathrm{O} 1 \mathrm{~s}$ intensity as a function of $h \nu$ is similar to that of the inverted $\beta$ curve, as discussed in the previous chapter (region I: Eq. (16a)). As $\phi_{1}$ decreases but still sits outside of the total reflection region, the curve shape becomes a more complex intermixing of the values of $\delta$ and inverted $\beta$ (region II: Eq. (16b)). When the incidence angle is in the total reflection region 


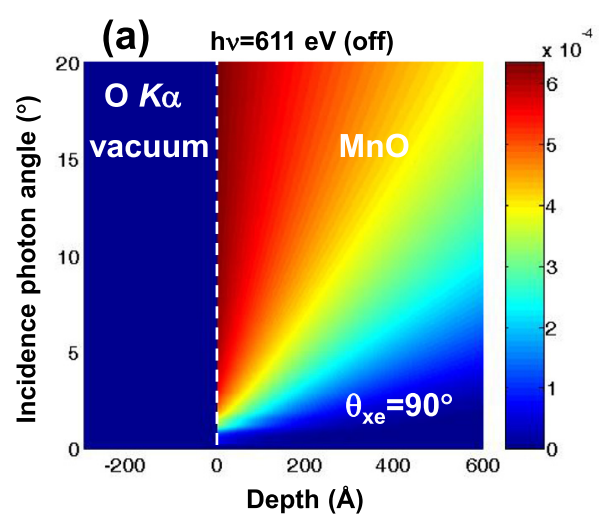

(c)

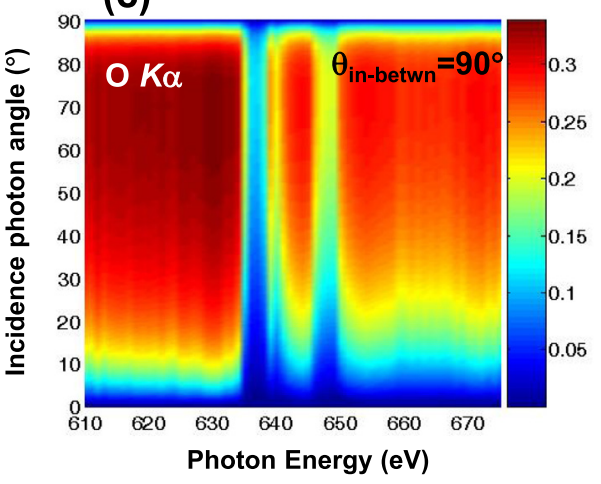

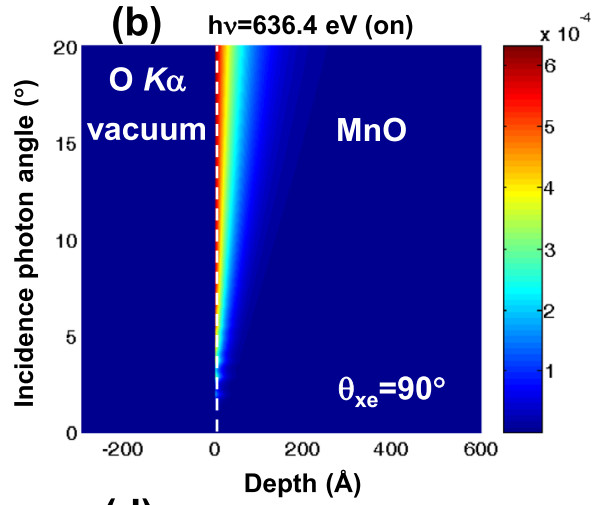

(d)

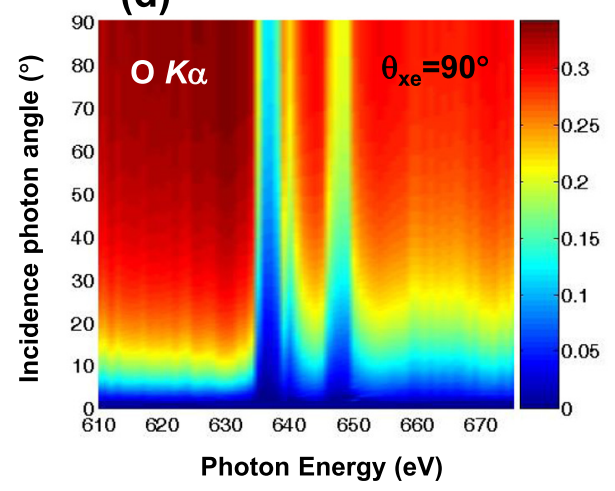

FIG. 6. O $K \alpha$ x-ray emission intensity depth profiles for $\mathrm{MnO}$ across the $\mathrm{Mn} 2 p$ edges as a function of incidence photon angle: (a) off-resonance and (b) onresonance. $\mathrm{O} K \alpha$ intensity plot vs. incidence photon angle and energy (c) for a $90^{\circ}$ angle between $\mathrm{x}$-ray incidence and exit and (d) for a $90^{\circ} \mathrm{x}$-ray exit angle (normal to the surface). (region III: Eq. (16c)), the attenuation term becomes almost constant and the shape of $I(h \nu)$ is largely determined by $\delta$.

Fig. 6 shows the calculated $\mathrm{O} K \alpha$ XES intensity in $\mathrm{MnO}$ as a function of $\phi_{1}$ on- and off-resonance near the Mn $2 p$ edge. Here (a), (b), and (c) are calculated using a constant "in-between angle" $\left(\phi_{\text {in-btwn }}=90^{\circ}\right)$ measured between the incident photon and emitting photon beams, while a normal take-off angle $\left(\phi_{\mathrm{xe}}=90^{\circ}\right)$ is used in (d). As expected, at the off-resonance energies, the probing depth for XES is significantly higher compared to that of PES (see, Figs. 5(c) and 6(a)). At $\phi_{1}=25^{\circ}$, the probing depth is about $800 \AA$, whereas that for PES is $\sim 8 \AA$. In contrast, on the resonance, the probing depth for XES and PES decreases down to about $80 \AA$ and $5 \AA$, respectively (Figs. 5(d) and 6(b)). As a result, XES is significantly more sensitive to the variations in optical parameters than PES, since the penetration depth for the excitation photons is comparable with that of the emitted photons. $\mathrm{O} K \propto \mathrm{XES}$ total intensity variation is further plotted in Figs. $6(c)$ and $6(d)$ as functions of incidence photon angle and photon energy around the Mn $2 p$ edge. The overall shapes are similar to that of the inverted $\beta$ curve for $5^{\circ}<\phi_{1}<85^{\circ}$ and $\phi_{\text {in-btwn }}=90^{\circ}$ (Fig. 6(c)), and for $5^{\circ}<\phi_{1}$ and normal take-off angle $\phi_{\mathrm{xe}}=90^{\circ}$ (Fig. 6(d)), resulting from the fact that the behavior of XES intensity is mostly governed by the $\mathrm{x}$-ray penetration depth. In the other region of incidence angles $\left(5^{\circ}>\phi_{1}\right.$ or $\phi_{1}>85^{\circ}$ and $\phi_{\text {in-btwn }}=90^{\circ} ; \phi_{1}<5^{\circ}$ and normal take-off angle $\phi_{\mathrm{xe}}=90^{\circ}$ ), the XES intensity is heavily perturbed by total reflection of incoming photons or emitted photons at the surface. Since conventional XES spectrometers usually use the constant $\phi_{\text {in-btwn }}$ scheme, the Fig. 6(c) calculation represents the geometry most directly related to the analysis of experimental data.
It should also be noted that the emitted photon may undergo substantial attenuation inside the material depending on its energy - self-absorption. For example, $\mathrm{O} \mathrm{K} \alpha$ may have enough energy to excite electrons into unoccupied $\mathrm{O}$ $2 p$-derived states in $\mathrm{MnO}$. This also is allowed for in the formalism we have outlined above.

\section{A multilayer sample as a standing-wave generator}

We now discuss $\mathrm{x}$-ray optical effects in a synthetic multilayer, or "superlattice" structure, which acts as a soft x-ray standing-wave generator. The more conventional hard $\mathrm{x}$-ray standing-wave technique involving Bragg reflection from crystal planes has been used extensively in studies of atomic structure at the $\AA$ scale, in both bulk crystals and near surfaces. ${ }^{15,16}$ However, beginning with work by Kim and Kortright $^{19}$ and by Yang et al., ${ }^{18,20}$ it has been shown that the standing-wave method can be extended into the soft x-ray regime $(\sim 0.5-1.5 \mathrm{keV})$ and it has subsequently been combined with high-resolution x-ray photoelectron spectroscopy to develop a non-destructive and element specific depth-resolved spectroscopic tool. ${ }^{18,20-32}$ This new soft $\mathrm{x}$-ray technique has several significant advantages: for soft $\mathrm{x}$-rays, the photoelectric cross sections are significantly larger than those in the hard X-ray regime, and the standing wave can be scanned through the layers, thus highlighting/de-highlighting a certain depth of a layer by either varying the photon incidence angle around the Bragg angle or varying the incidence angle around the Bragg condition, as noted earlier. Beyond this, by making one of the layers in the sample structure in a wedge profile, it is possible to minimize the number of free parameters to fit, by exploiting the nearly perfect "phase pinning" of the standing waves with respect to the standing-wave 
generator. In this manner, the standing wave can be scanned through the sample in the direction perpendicular to the sample plane, thus providing additional depth-selectivity to the normal SW experiment. This has been termed the "SWEDGE" method.

To generate high contrast standing waves, a significant difference in optical constants between the two components of the bilayers making up the multilayer mirror is necessary. These optical constants are directly related to the scattering form factor, which defines the interaction between the incident photon and target atom. The scattering form factor is furthermore proportional to the atomic number of the target atom. ${ }^{46}$ Therefore, a higher atomic number contrast between the two components leads in general to a higher-modulated standing wave, as is for example, the case with the $\mathrm{B}_{4} \mathrm{C} / \mathrm{W}$ or $\mathrm{Si} / \mathrm{Mo}$ pairs that are often used for multilayer $\mathrm{x}$-ray mirrors. The conditions for Bragg reflection and the nature of the standing wave generated have been introduced previously (see, e.g., Eqs. (34) and (35)). (a)

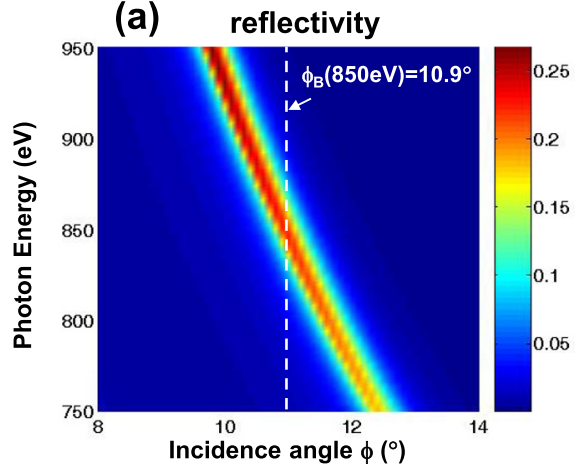

(c)

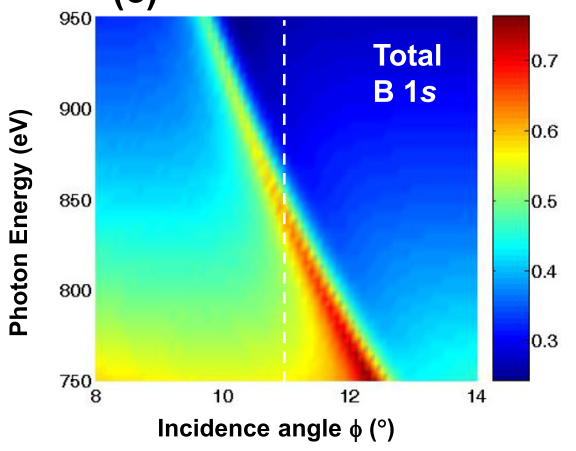

(e)

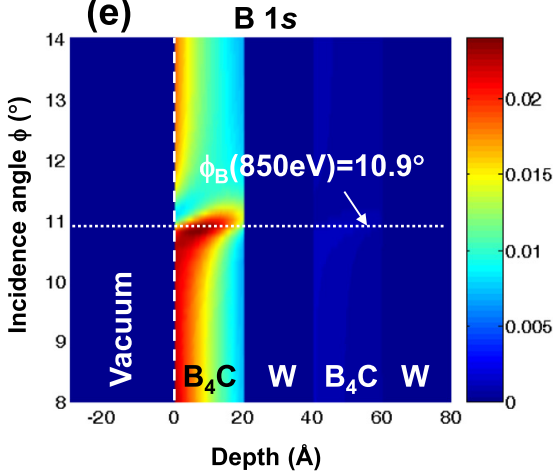

(g)

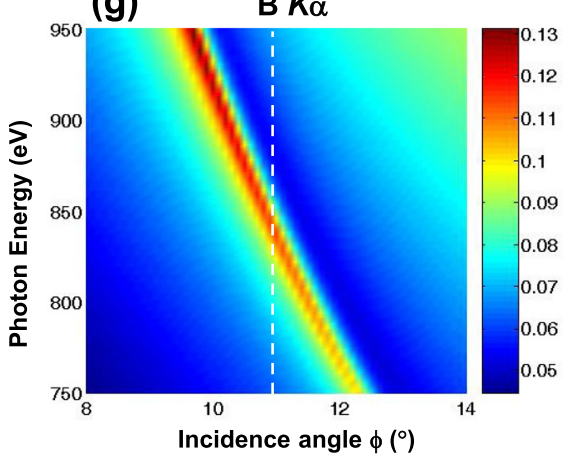

(b) normalized $|E|^{2}$

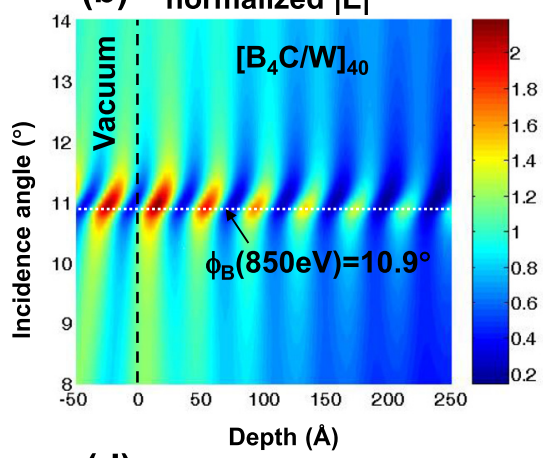

(d)

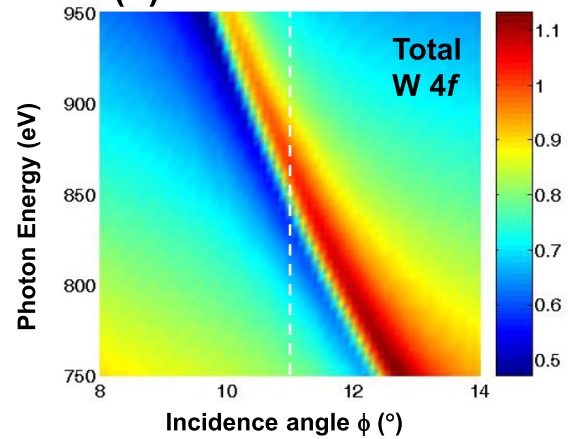

(f)

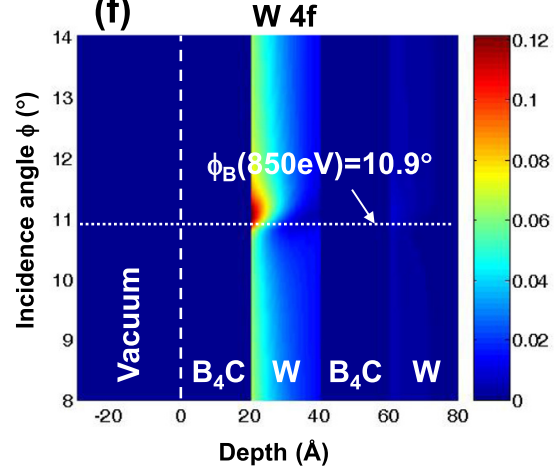

(h)

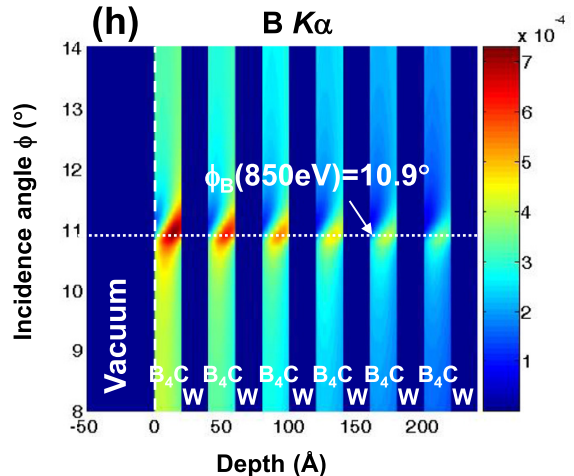

FIG. 7. X-ray optical calculations for a multilayer standing wave generator [20 ̊ B4C/ $20 \AA \mathrm{W}]_{40}$ exposed to $p$-polarized light: (a) Reflectivity vs. incidence photon angle and energy. The dashed vertical line represents Bragg angle $\phi_{B}=10.9^{\circ}$ corresponding to a reference energy of $h \nu=850 \mathrm{eV}$. (b) The depth profile of electric field strength normalized to an incident wave of unity as a function of incidence photon angle for $h \nu=850 \mathrm{eV}$. The horizontal line represents the Bragg angle of $\phi_{B}=10.9^{\circ}$. Depthintegrated photoelectron intensities for (c) B $1 s$ and (d) W $4 f$ vs. incidence photon angle and energy at a $90^{\circ}$ take-off angle (normal to the surface). Depth-resolved photoelectron intensities as a function of incident $\mathrm{x}$-ray angle with $850 \mathrm{eV}$ excitation for (e) B $1 s$ and (f) W $4 f$. (g) Depth-integrated x-ray emission intensity for B $K \alpha$ vs. incidence photon angle and energy with a $90^{\circ}$ in-between angle. Vertical line represents Bragg angle $\phi_{\mathrm{B}}=10.9^{\circ}$ corresponding to $h \nu=850 \mathrm{eV}$. (h) Depthresolved $\mathrm{B} K \alpha \mathrm{x}$-ray emission as a function of incidence photon angle. 
We will focus on the first-order Bragg angle at which more than $10 \%$ reflectivity can be readily achieved in the soft $\mathrm{x}$-ray region with a typical $\mathrm{B}_{4} \mathrm{C} / \mathrm{W}$ multilayer having 40-60 bilayer periods, a 20-60 small but reasonable interdiffusion at the interfaces of $\sigma<5 \AA$. As a specific example, Fig. 7(a) plots the reflectivity for a multilayer of form $\left[20 \AA \mathrm{B}_{4} \mathrm{C} / 20 \AA \mathrm{W}\right]_{40}$, grown on an oxidized substrate of $2500 \AA \mathrm{SiO}_{2} / \mathrm{Si}$ as functions of photon energy over a range of 750 to $950 \mathrm{eV}$ and incidence angle from $0^{\circ}$ to $15^{\circ}$ that spans the first-order Bragg angle of the multilayer at about $11^{\circ}$. Of course, for all energies, the reflectivity goes to 1 for zero incidence angle. As the photon energy decreases, Bragg reflectivity and electric field contrast decrease and the Bragg angle shifts to higher values as seen in Fig. 7(a). It should be noted that the high reflectivity in the total reflection regime near zero incidence angle does not give rise to a large standing-wave modulation of the electric field in the multilayer, but only in vacuum because the origin of high reflectivity at the Bragg angle is fundamentally different from that of total reflection at grazing incidence.

Fig. 7(b) shows a plot of the electric field intensity modulation as functions of the depth and incidence angle for a photon energy of $850 \mathrm{eV}$ and the same multilayer as in 7 (a). The calculated electric field intensities are all normalized to the incident beam intensity. Deviating by $1^{\circ}$ from the Bragg angle $\left(\phi_{\mathrm{B}}=10.9^{\circ}\right)$ gives rise to a dramatic reduction in the standing-wave electric field intensity. Values of reflectivity at the Bragg angle $\phi_{\mathrm{B}}(23 \%), \phi=9.9^{\circ}(1.6 \%)$ and $\phi=11.9^{\circ}$ $(0.97 \%)$ correspond to minimum-maximum electric field modulations of $0.271 \sim 2.189$ or 1.918 relative to the incident intensity for $\phi_{\mathrm{B}}, 0.763 \sim 1.269$ or 0.506 for $\phi=9.9^{\circ}$ and $0.812 \sim 1.2067$ or 0.395 for $\phi=11.9^{\circ}$ in vacuum. The relative numbers quoted here are furthermore very close to those given by $\pm \sqrt{ } R$, as discussed in the text following Eq. (37). The depth dependence shown here implies that the modulation of the electric field strength at the Bragg angle is strong enough to highlight a specific buried region, which is located as deep as $20 \sim 30 \AA$ below the surface for photoemission (with the limit being set by the IMFP for photoelectrons), and up to over $1000 \AA$ for $x$-ray emission (with the limit set by the penetration depth of the x-rays). Figs. 7(c), $7(\mathrm{~d})$, and $7(\mathrm{~g})$ further plot the total photoemission (B $1 s$ and $\mathrm{W} 4 f)$ and total $\mathrm{x}$-ray emission $(\mathrm{B} K \alpha)$ intensity variation as functions of photon energy and incidence angle, where normal take-off angle and constant in-between angle of $90^{\circ}$ have been used for photoelectron and x-ray emission, respectively. In the region between $\phi=0^{\circ} \sim 4^{\circ}$, the spectral intensities increase exponentially with the increasing incidence angle because this region corresponds to total reflection where $\Lambda_{\mathrm{pe}}$ is comparable with the x-ray penetration depth that increases dramatically with increasing $\phi$. When the incidence angle increases beyond the total reflection region, the PES intensities gradually decrease due to the interaction factor $A(\eta, \xi)$, while XES intensities continue to increase until $\phi \sim 85^{\circ}$. As for $\phi>85^{\circ}$, the emitting photon is in the internal total reflection region (strong self-absorption) and the XES intensity sharply decreases with increasing $\phi$ (see Fig. 6(c)). Figs. 7(c), 7(d), and 7(g) show the pronounced PES and XES intensity variations, respectively, as one scans $\phi$ through the Bragg angle: a rocking curve scan. The depth profiles of emitted intensity corresponding to these two rocking curves are further plotted in Figs. 7(e), 7(f) (PES), and 7(h) (XES). It is clearly seen that the XES intensity profile is more closely connected with the electric field strength modulation than that of PES due to the greater attenuation lengths of $\mathrm{x}$-rays, so that XES can probe deeper-buried layers or interfaces.

We also note that, in Figs. 7(a), 7(c), 7(d), and 7(g), the small oscillations along the maxima curves are due to Kiessig fringes, as described via Eq. (36) and (37), and discussed further below.

As a final comment, Fig. 7(b) further demonstrates that the maximum of the electric field intensity in such a standing-wave scan shifts by very close to one half of the period of the bilayer in the mirror in going from angles below the Bragg angle to angles above the Bragg angle. The same is found to be true if we sit at a fixed angle and scan photon energy through the Bragg condition for the multilayer. Thus, the region enhanced by the standing-wave maximum shifts in vertical position by one half of the bilayer period in doing either a rocking curve or a photon energy scan.

\section{A multilayer mirror with wedge profile sample}

We now turn to the situation of a multilayer substrate with a wedge-profile sample on top, as appropriate to the SWEDGE method mentioned above. We consider a sample consisting of $15 \AA$ of $\mathrm{Fe}$ on top of a $\mathrm{Cr}$ wedge varying from 35 to $135 \AA$ in thickness, in turn grown on top of the same $\mathrm{B}_{4} \mathrm{C} / \mathrm{W}$ mirror as considered for the results in Fig. 8. For a photon energy of $850 \mathrm{eV}$, Figs. 8 (a)-8(d) plot (a) reflectivity as a function of the $\mathrm{x}$-ray spot position along the $\mathrm{Cr}$ thickness and incidence angle, and, at an incidence angle of $\phi_{B}=10.9^{\circ}$, (b) the depth-resolved electric field strength as a function of $\mathrm{Cr}$ thickness, and (c) and (d) the photoemission intensity from $\mathrm{Fe} 2 p$ and $\mathrm{Cr} 2 p$, respectively. Not only do the maximum values of reflectivity $\left(R_{\max }\right)$ for $\phi>9^{\circ}$ change by less than $10 \%$ as the $\mathrm{Cr}$ thickness is scanned, but the position of the maximum is effectively pinned at the angle associated with the first order Bragg condition of $\phi_{\mathrm{B}}=10.9^{\circ}$. Fig. 8(b) further shows that there is very little change in phase and amplitude of the SW as the Cr thickness is varied. Since the incidence angle and the bilayer period determine the standing wave period (see, Eq. (34)), the constant $R_{\max }$ position further indicates that the standing wave phase is pinned, thus enabling depth-resolved characterization of buried layers or interfaces. This phase pinning is valid only when the x-ray penetration length is longer than the total thickness of standing-wave generating multilayer and the Bragg angle is outside of the total reflection region. The "SWEDGE" technique using this phase pinning has a couple of significant advantages for depth-resolved SW studies: (a) no scan of energy or angle is necessary to have the standing wave scan vertically through the layers, (b) the SW modulation amplitude varies significantly less than those for the energy or angle scan, (c) a wedge scan can involve several periods of the SW passing through the sample, thus effectively enlarging the dataset and yielding very nearly sinusoidal variations 


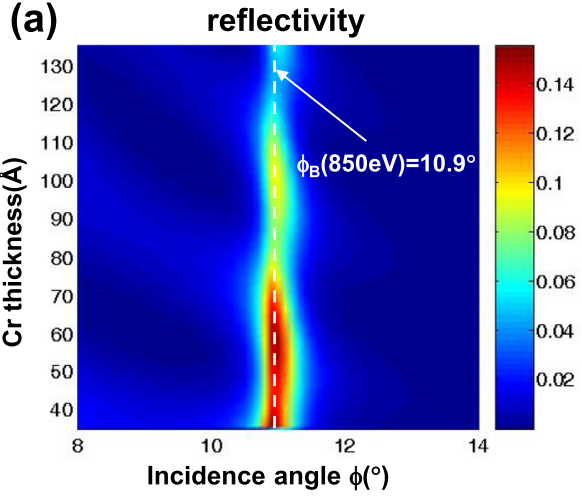

(c)

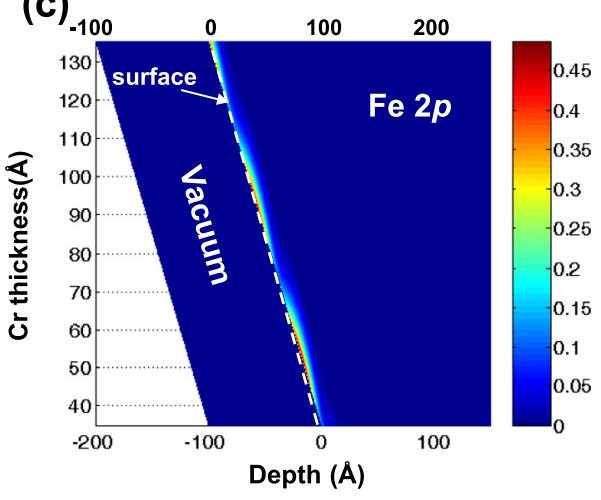

(e)

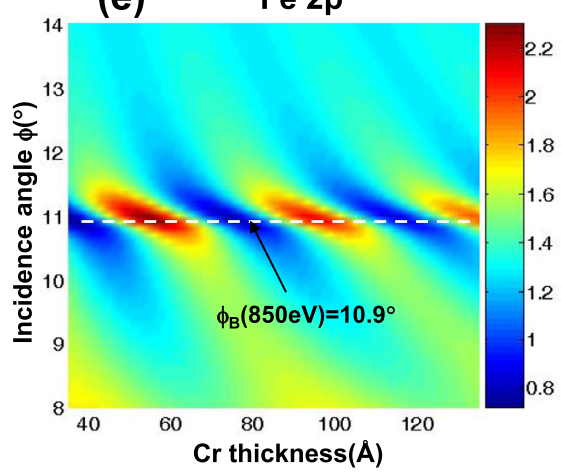

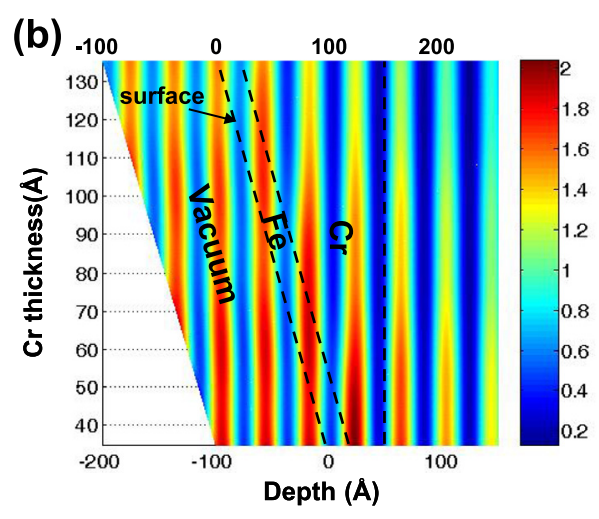

(d)

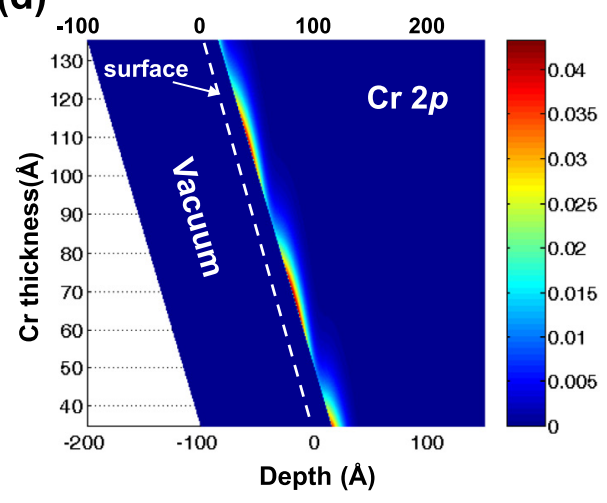

(f)

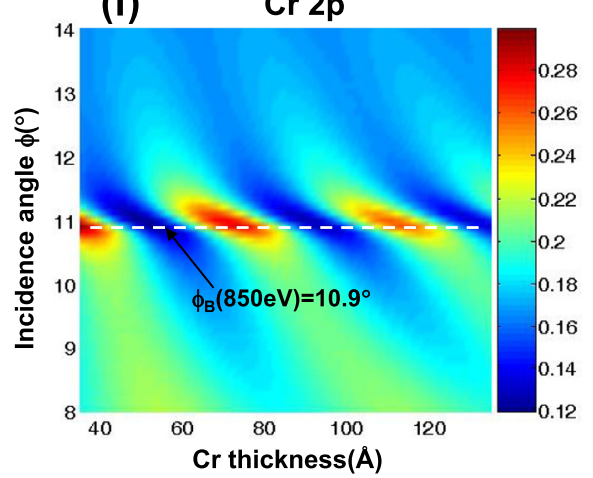

FIG. 8. Calculations for a wedge-profile sample on top of a multilayer mirror consisting of $15 \AA \mathrm{Fe} / \mathrm{a} 35 \sim 135 \AA \mathrm{Cr}$ wedge/a multilayer of form [20 $\AA \mathrm{B}_{4} \mathrm{C} /$ $20 \AA \mathrm{W}]_{40}$ : (a) $3 \mathrm{D}$ contour plot of reflectivity vs. incidence photon angle and $\mathrm{Cr}$ thicknesses. Note that the reflectivity peak at Bragg angle $\phi_{\mathrm{B}}=10.9^{\circ}$ oscillates as a function of $d_{\mathrm{Cr}}$ thus exhibiting local maxima at $d_{\mathrm{Cr}} \sim 65,105$, and $145 \AA$, and local minima $d_{\mathrm{Cr}} \sim 45,85$, and $125 \AA$. These correspond to $d_{\mathrm{Fe}}+d_{\mathrm{Cr}}=80,120$, and $160 \AA\left(n \lambda_{\mathrm{sw}}\right)$ for local maxima, and 60,100 , and $140 \AA$ $\left(\left(n+\frac{1}{2}\right) \lambda_{\mathrm{sw}}\right)$ for local minima, which implies that the constructive interference happens and the amplitude of standing wave becomes stronger when the thickness of $\mathrm{Fe} / \mathrm{Cr}$ overlayer is the multiple of standing wave period while vice versa when the $\mathrm{Fe} / \mathrm{Cr}$ layer is half periods thick. Vertical line represents Bragg angle $\phi_{\mathrm{B}}=10.9^{\circ}$ again corresponding to $h \nu=850 \mathrm{eV}$. (b) Depth profile of electric field strength depth as a function of $\mathrm{Cr}$ thickness. Note the nearly constant position of the SW maximum in depth relative to the multilayer, an indication of the phase pinning. (c) $\mathrm{Fe} 2 p$ and (d) $\mathrm{Cr}$ $2 p$ photoelectron intensity profiles as a function of $\mathrm{Cr}$ thicknesses, and (e) and (f) their integrated intensity $3 \mathrm{D}$ contour plot vs. incidence photon angle and $\mathrm{Cr}$ thicknesses. that can be more quantitatively analyzed for depth profile information. These advantages effectively reduce the number of fitting parameters and enable significantly more reliable depth-resolved characterization with higher spatial resolution than is possible with either angle scans (rocking curves) or photon energy scans. The depth-resolved plots of Fe $2 p$ and $\mathrm{Cr} 2 p$ intensity variation vs. Cr thickness shown in Figs. 8(c) and $8(\mathrm{~d})$ indicate that the interface between $\mathrm{Fe}$ and $\mathrm{Cr}$ will be emphasized at $d_{\mathrm{Cr}} \approx 72 \AA$ and $112 \AA$ where $\mathrm{Cr} 2 p$ intensities form local maxima, while it will be de-emphasized at $d_{\mathrm{Cr}} \approx$ $52 \AA, 92 \AA$, and $132 \AA$ where Cr $2 p$ intensities form local minima. It should be noted that $\mathrm{Fe} 2 p$ photoemission intensity does not have a minimum (maximum) at the positions where the interface between $\mathrm{Fe}$ and $\mathrm{Cr}$ is emphasized (de-emphasized) because the thickness of the Fe layer is $15 \AA$. That is, Fe $2 p$ intensity maxima (minima) occur with about a $15 \AA$ phase delay with respect to the $\mathrm{Cr} 2 p$ minima (maxima), at $d_{\mathrm{Cr}} \approx 57 \AA$ and $97 \AA$. Depth-integrated Fe and $\mathrm{Cr} 2 p$ total photoemission intensity plots as functions of $\mathrm{Cr}$ thickness and incidence angle are displayed in Figs. 8(e) and 8(f). It is obvious that the intensity modulations reach their maxima at the Bragg angle and that $\mathrm{Fe} 2 p$ maxima (or minima) do not coincide with the $\mathrm{Cr} 2 p$ minima (or maxima), but that that maxima are displaced by very nearly $15 \AA$ from one another, consistent with the $\mathrm{Fe}$ and $\mathrm{Cr}$ layers being displaced vertically with respect to one another.

Analyzing experimental $\mathrm{Fe} 2 p$ and $\mathrm{Cr} 2 p$ photoemission data from this sample via a trial-and-error use of the YXRO program $^{21}$ has in fact permitted deriving the detailed atomic concentration profiles through the $\mathrm{Fe} / \mathrm{Cr}$ interface, with the final results of this study being summarized on the left side of Fig. 9.

Depth-resolved x-ray emission spectroscopy using the SWEDGE method can also provide complementary information on buried layers/interfaces due to the longer $\mathrm{x}$-ray attenuation lengths of the emitted x-rays as compared to photoelectrons. Fig. 10 plots the calculated Fe $L \alpha$ ((a) and (c)) and $\mathrm{Cr} L \alpha$ ((b) and (d)) depth profiles and integrated x-ray intensities for the same sample and multilayer mirror. Compared to the photoemission case, the Fe $L \alpha$ modulations of 


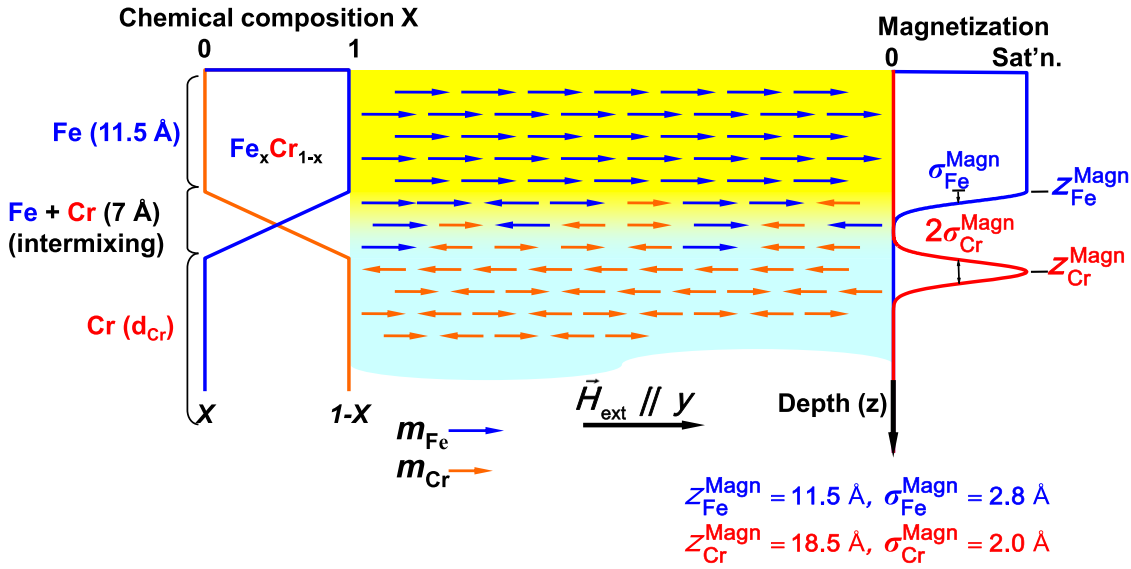

FIG. 9. Schematic diagram of a $\mathrm{Fe} / \mathrm{Cr}$ layer with interdiffused region and magnetic moment distribution profile in a sample with configuration $15 \AA \mathrm{Fe} /$ $35 \sim 135 \AA$ Cr-wedge/[20 $\AA \mathrm{B} 4 \mathrm{C} / 20 \AA \mathrm{W}]_{40}$. The parameters here were derived from experimental SWEDGE measurements, as described in Ref. 21.

Figs. 10(a) and 10(c) are comparable to those of Fe $2 p$ photoemission (Figs. 8(c) and 8(e)), while and analogous comparison for $\mathrm{Cr} L \alpha$ (Fig. 10(d)) yields significantly smaller modulations than those of $\mathrm{Cr} 2 p$ (Fig. 8(f)). This is because the x-ray attenuation length $(\sim 190 \AA$ for the $\mathrm{Cr} L \alpha$ emission line in $\mathrm{Cr}$ ) is significantly larger that the photoelectron IMFP, and thus $\mathrm{Cr} L \alpha$ effectively samples several SW periods, thus reducing the SW modulations. More quantitatively, Fig. 10(b) shows that $\mathrm{Cr} L \alpha$ total intensity samples at least three periods of the SW strongly. Also, since increasing $d_{\mathrm{Cr}}$ leads to an increased $\mathrm{Cr}$ sampling depth, the integrated $\mathrm{Cr} L \alpha$ intensity is expected to increase with increasing $d_{\mathrm{Cr}}$. Excellent agreement between calculations of this type and experiment is in fact reported in Ref. 26, to which the reader is referred for more details.

Beyond determining concentration profiles, elementspecific magnetization of each species can be determined by combining the SWEDGE method and magnetic circular dichroism (MCD) in photoemission or x-ray emission. The normalized MCD intensity is determined from the formula

$$
I_{\mathrm{MCD}}=\frac{I_{\mathrm{LCP}}-I_{\mathrm{RCP}}}{\left[I_{\mathrm{LCP}}+I_{\mathrm{RCP}}\right] / 2},
$$

where $I_{\mathrm{LCP}}$ and $I_{\mathrm{RCP}}$ are photoemission/x-ray emission intensities excited by left-and right-circularly polarized radiation (LCP and RCP), respectively, and they can be written as

$$
\begin{aligned}
I_{\mathrm{LCP}}\left(\phi_{i n c}, d_{\mathrm{Cr}}\right)= & C \int\left[1+\mu_{\mathrm{LCP}}(z)\right]\left|\vec{E}\left(\phi_{i n c}, z, d_{\mathrm{Cr}}\right)\right|^{2} \\
& \times e^{-z / \Lambda \sin \phi} \rho(z) \frac{d \sigma_{x}}{d \Omega} d z \\
I_{\mathrm{RCP}}\left(\phi_{i n c}, d_{\mathrm{Cr}}\right)= & C \int\left[1+\mu_{\mathrm{RCP}}(z)\right]\left|\vec{E}\left(\phi_{i n c}, z, d_{\mathrm{Cr}}\right)\right|^{2} \\
& \times e^{-z / \Lambda \sin \phi} \rho(z) \frac{d \sigma_{x}}{d \Omega} d z
\end{aligned}
$$

(a)

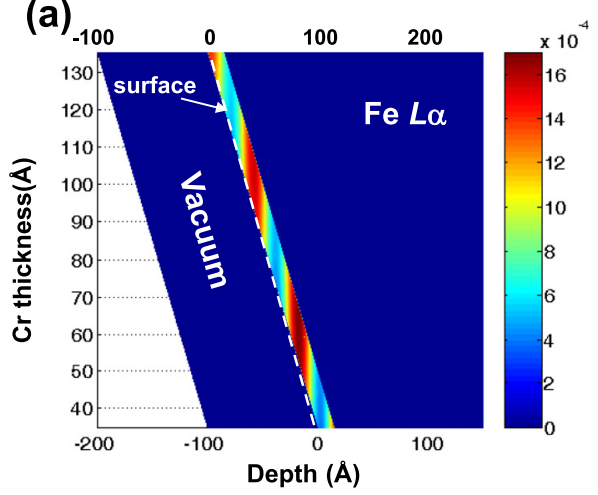

(c)

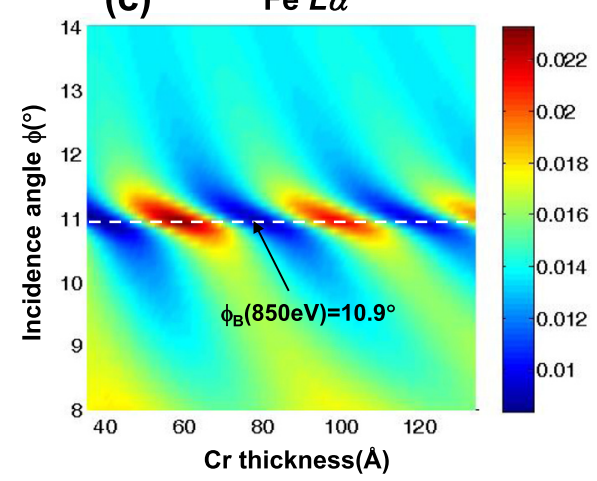

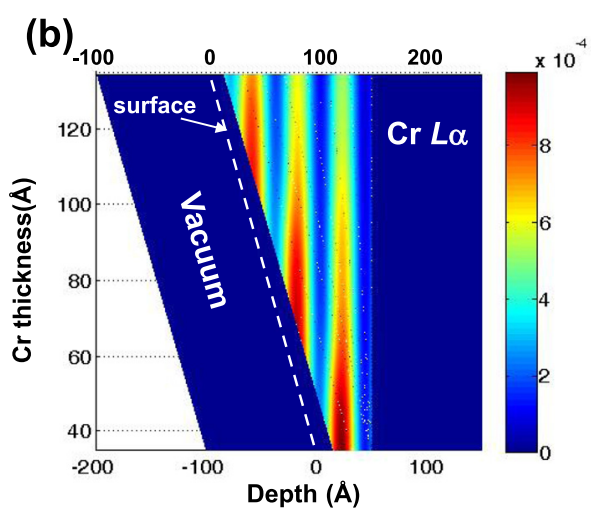

(d)

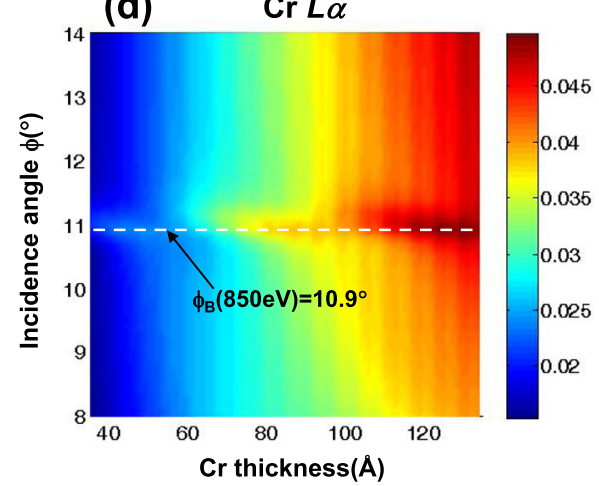

FIG. 10. Calculations for a wedgeprofile sample on top of a multilayer mirror consisting of $15 \AA \mathrm{Fe} / 35 \sim 135 \AA$ Cr-wedge/[20 $\left.\AA \mathrm{B}_{4} \mathrm{C} / 20 \AA \mathrm{W}\right]_{40}$ : (a) $\mathrm{Fe}$ $L \alpha$ and (b) $\operatorname{Cr} L \alpha$ x-ray emission intensity profiles as a function of $\mathrm{Cr}$ thicknesses, and (c) and (d) their integrated intensity vs. incidence photon angle and $\mathrm{Cr}$ thicknesses. The standing-wave modulation is much reduced for $\mathrm{Cr}$ in (d) due to the greater emission depth of $\mathrm{Cr} L \alpha$ and the greater thickness of the wedge compared to the period of the SW. 
where $C$ is a constant, $\mu_{\mathrm{LCP}}(z)$ and $\mu_{\mathrm{RCP}}(z)$ are the depth dependent contributions of a given atomic type to the magnetic dichroism, $\left|\vec{E}\left(\phi_{i n c}, z, d_{\mathrm{Cr}}\right)\right|^{2}$ is the electric field intensity of the SW for a given incidence angle $\phi_{\text {inc }}$, at a given depth $z$ and at a given $\mathrm{Cr}$ thickness $d_{\mathrm{Cr}}, \Lambda$ is an attenuation length ( $\Lambda_{p e(x e)}$ in earlier equations), and $\phi$ is an internal emission angle $\left(\phi_{p e(x e)}^{0}\right.$ in prior equations) of emitted photoelectrons or $\mathrm{x}$-ray photons, depending on whether we are considering photoemission or x-ray emission. To a good approximation, $\mu_{\mathrm{LCP}}(z)$ and $\mu_{\mathrm{RCP}}(z)$ can also be related to one another via a proportion of the form $\mu_{\mathrm{LCP}}(z)-\mu_{\mathrm{RCP}}(z)=\kappa m(z)$, where $\kappa$ is a constant and $m(z)$ is the depth-dependent magnetization (magnetic moment) per atom due to a specific atomic type, e.g., Fe and $\mathrm{Cr}$ here. Now, Eq. (38) becomes

$$
\begin{aligned}
\mathrm{I}_{\mathrm{MCD}}\left(\phi_{i n c}, d_{\mathrm{Cr}}\right) & =\frac{2 \int\left[\mu_{\mathrm{LCP}}(z)-\mu_{\mathrm{RCP}}(z)\right]\left|\vec{E}\left(\phi_{i n c}, z, d_{\mathrm{Cr}}\right)\right|^{2} e^{-z / \Lambda \sin \phi} \rho(z) \frac{d \sigma_{x}}{d \Omega} d z}{\int\left[2+\mu_{\mathrm{LCP}}(z)+\mu_{\mathrm{RCP}}(z)\right]\left|\vec{E}\left(\phi_{i n c}, z, d_{\mathrm{Cr}}\right)\right|^{2} e^{-z / \Lambda \sin \phi} \rho(z) \frac{d \sigma_{x}}{d \Omega} d z} \\
& \cong \frac{\int\left[\mu_{\mathrm{LCP}}(z)-\mu_{\mathrm{RCP}}(z)\right]\left|\vec{E}\left(\phi_{i n c}, z, d_{\mathrm{Cr}}\right)\right|^{2} e^{-z / \Lambda \sin \phi} \rho(z) \frac{d \sigma_{x}}{d \Omega} d z}{\int\left|\vec{E}\left(\phi_{i n c}, z, d_{\mathrm{Cr}}\right)\right|^{2} e^{-z / \Lambda \sin \phi} \rho(z) \frac{d \sigma_{x}}{d \Omega} d z},
\end{aligned}
$$

where the last approximate equality holds if we assume that $\mu_{\mathrm{LCP}}(z)$ and $\mu_{\mathrm{RCP}}(z)$ are much less than unity. At this point, it is important to note that, if the contribution of each atom of a given type to magnetic dichroism is the same, regardless of its position inside a given layer or in an interface between two layers, it is equivalent to both $\mu_{\mathrm{LCP}}(z)$ and $\mu_{\mathrm{RCP}}(z)$ being independent of $z$ (or constants). In this case, Eq. (40) is reduced to $\mathrm{I}_{\mathrm{MCD}}\left(\phi_{\text {inc }}, d_{\mathrm{Cr}}\right) \cong \mu_{\mathrm{RCP}}-\mu_{\mathrm{RCP}}=\kappa m$. This implies that MCD will be constant during a SW scan in $z$ or, in fact, also a rocking curve scan in $\phi_{i n c}$, unless the contribu- tions of a given atom to dichroism as a function of depth differ from the concentration profile of that atom. An alternative and very useful way of looking at this is that if the MCD is found to vary during a SW scan or rocking curve measurement, the contribution of a given atom to magnetic dichroism is not constant through the layer or through one or both of the interfaces bounding the layer containing that atom.

To demonstrate this, an intermixing between $\mathrm{Fe}$ and the $\mathrm{Cr}$ wedge layer that produces non-uniform magnetization (a)

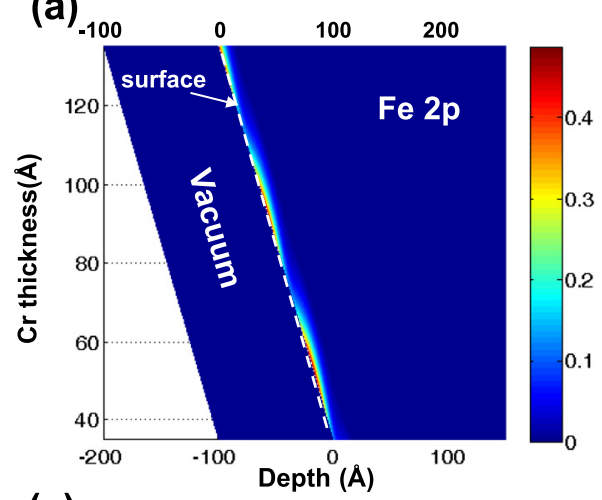

(c)

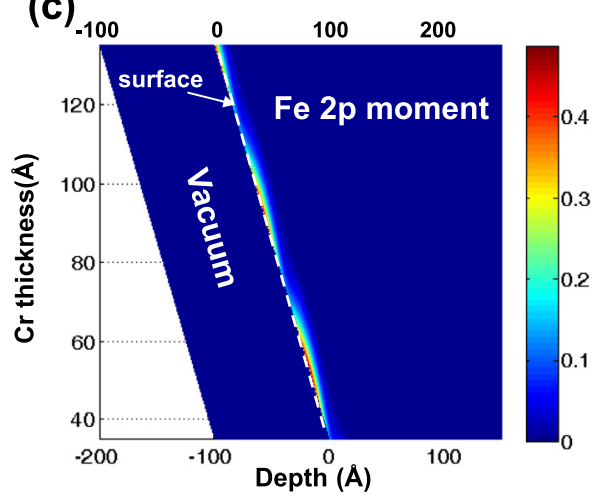

(b)

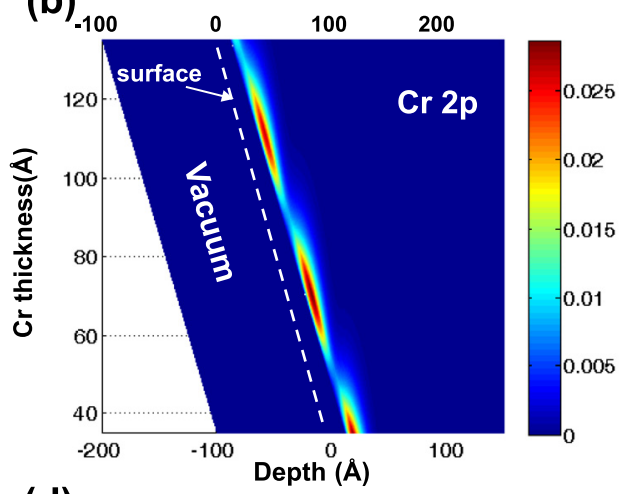

(d)

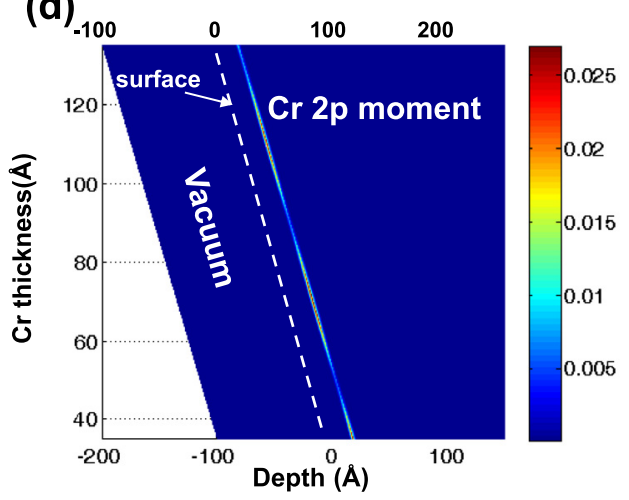

FIG. 11. Calculations for a wedgeprofile sample on top of a multilayer mirror consisting of $15 \AA \mathrm{Fe} / 35 \sim 135 \AA$ Cr-wedge/[20 $\left.\AA \mathrm{B}_{4} \mathrm{C} / 20 \AA \mathrm{W}\right]_{40}$, but for which the $\mathrm{Fe} / \mathrm{Cr}$ interface is linearly interdiffused and has a Gaussian profile of element-specific magnetization (magnetic moment), as shown in Fig. 9: (a) Fe $2 p$ and (b) $\mathrm{Cr} 2 p$ photoelectron intensity profiles as a function of $\mathrm{Cr}$ thicknesses. Horizontal vertical line represents Bragg angle $\phi_{\mathrm{B}}=10.9^{\circ}$ corresponding to $h \nu=850 \mathrm{eV}$. (c) Fe $2 p$ and (d) $\mathrm{Cr} 2 p$ photoelectron intensity profiles that represent the magnetic moments of (c) $\mathrm{Fe}$ and (d) $\mathrm{Cr}$ as a function of $\mathrm{Cr}$ thicknesses. 
(a)

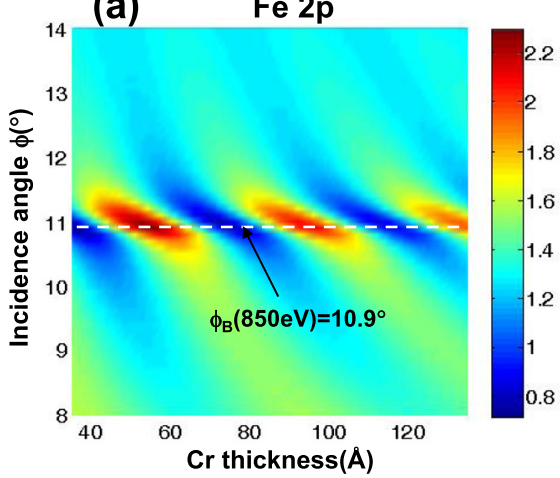

(c) Fe 2p moment

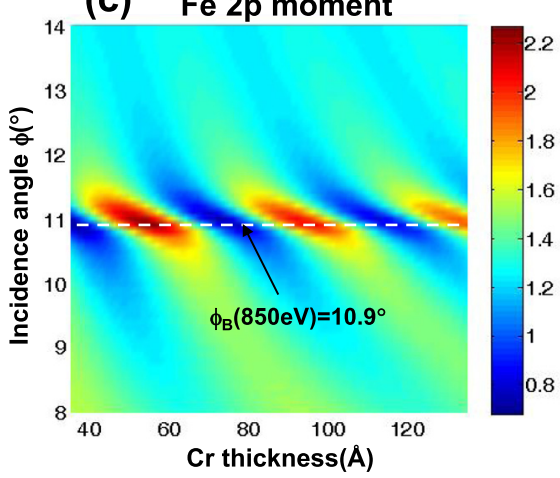

(e) Fe 2p MCD

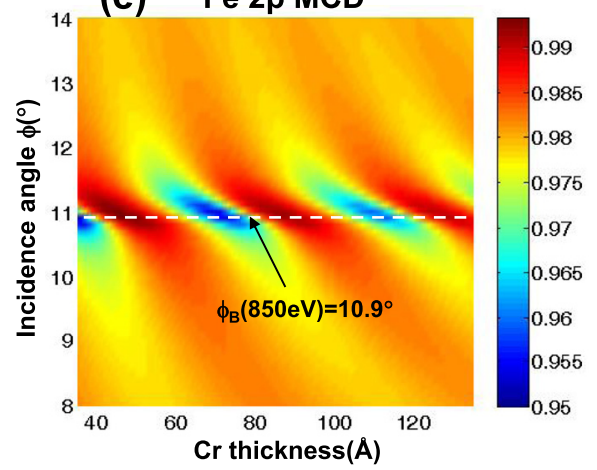

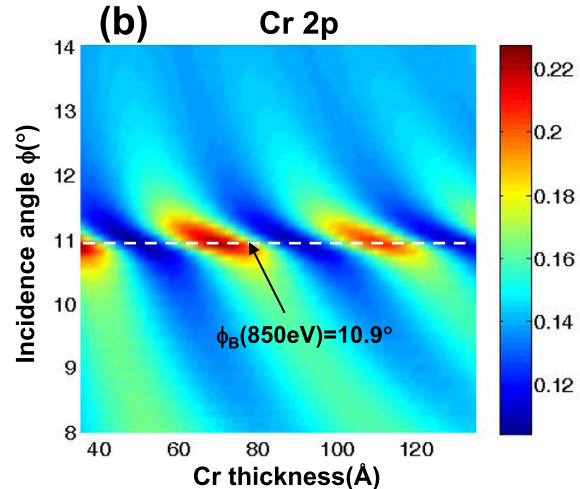

(d)

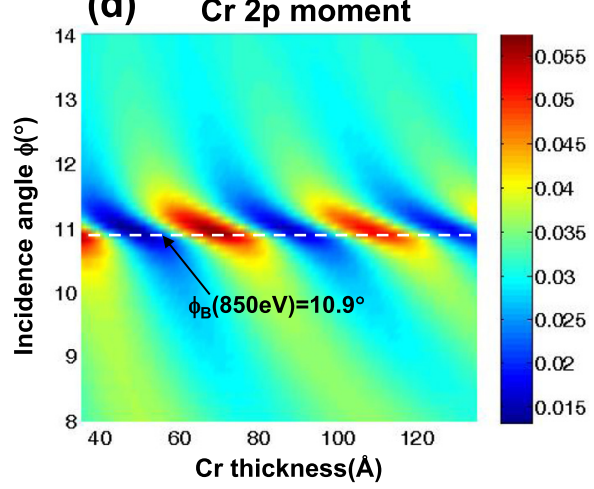

(f) $\mathrm{Cr} 2 \mathrm{p} \mathrm{MCD}$

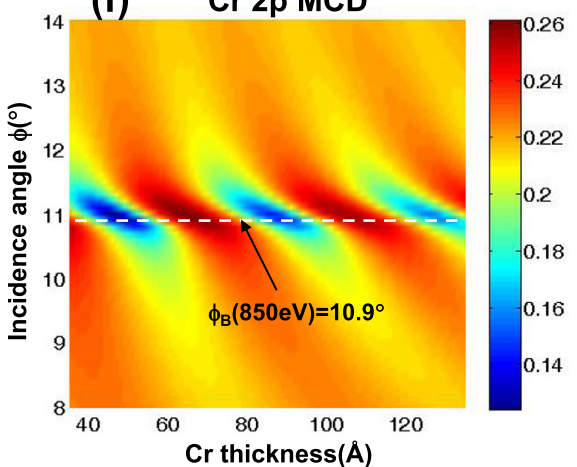

FIG. 12. (a) Fe $2 p$ and (b) $\mathrm{Cr} 2 p$ 3D integrated intensity plot vs. incidence photon angle and Cr thicknesses based on Figs. 11(a) and 11(b). Integrated contribution to the magnetic moments of (c) $\mathrm{Fe} 2 p$ (d) $\mathrm{Cr} 2 p$ based on Figs. 11(c) and 11(d), again as a function of incidence angle and Cr thickness. The final MCD contour plot, against the same coordinates, for (e) $\mathrm{Fe} 2 p$ and (f) $\mathrm{Cr} 2 p$. depth profiles, as derived previously from experiment ${ }^{21}$ is assumed, as shown in Fig. 9. The linearly interdiffused region spans over $7 \AA$, and the associated magnetization profiles that have been derived by analyzing experimental data with Eq. (40) (Ref. 21) show that the Fe magnetic moment is uniform in the non-diffused region while it rapidly decreases with the half-width-half-maximum (HWHM) $2.8 \AA$, and the $\mathrm{Cr}$ moment is zero all over the place except the region just underneath the intermixed region $(z=18.5 \AA)$ with $\mathrm{HWHM}=2 \AA$. For further insight into these results, we show calculations related to MCD measurements for $\mathrm{Fe}$ and $\mathrm{Cr} 2 p$ photoemission in Figs. 11 and 12 and for $\mathrm{Fe} L \alpha$ and $\mathrm{Cr}$ $L \alpha$ X-ray emission in Figs. 13 and 14. The depth-resolved intensities as a function of $\mathrm{Cr}$ thickness are obtained from the average of LCP and RCP excitation, as $\left[I_{\mathrm{LCP}}+I_{\mathrm{RCP}}\right] / 2$. Depth-resolved MCD signals proportional to the elementspecific magnetization (magnetic moment) are also shown. The final MCD profiles $\left[I_{\mathrm{LCP}}-I_{\mathrm{RCP}}\right] / I_{\mathrm{ave}}$ for photoemission are plotted in Figs. 12(e) and 12(f) and for x-ray emission in Figs. 14(e) and 14(f), with these showing significant modula- tions as functions of angle (rocking curve) and $d_{\mathrm{Cr}}$. Such modulations of MCD in SWEDGE method have been clearly observed in experiments on $\mathrm{Fe} / \mathrm{Cr}$ in both photoemission ${ }^{21}$ and in $\mathrm{x}$-ray emission ${ }^{26}$ thus showing that the magnetic moments in $\mathrm{Fe}$ and $\mathrm{Cr}$ are not uniform across the layers due to intermixing at the $\mathrm{Fe} / \mathrm{Cr}$ interface.

The SWEDGE method can also be very powerful for extracting the matrix-element-weighted depth-resolved valence band (VB) density of states (DOS) from layers or interfaces of interest, ${ }^{25,30,31}$ and we now illustrate this from a theoretical point of view for the same Fe layer on a $\mathrm{Cr}$ wedge. In contrast to core levels, the VB spectra from each layer will in general overlap each other in the binding energy regime from the Fermi level to approximately $15-20 \mathrm{eV}$, and it can thus be very hard to extract the DOS from each layer or interface with conventional photoemission spectroscopy. But if we now look at this from the point of view of a SWEDGE measurement, the VB photoelectron intensity at a certain kinetic energy $E_{\text {kin }}$ in a given $\mathrm{Cr}$ wedge thickness $d_{\mathrm{Cr}}$ can be written as 
(a)

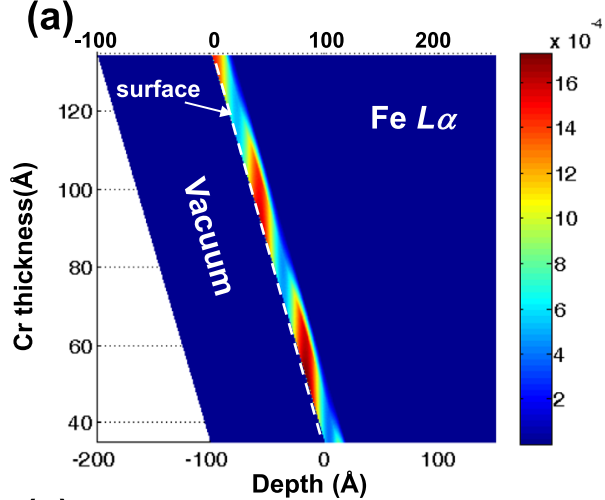

(c)

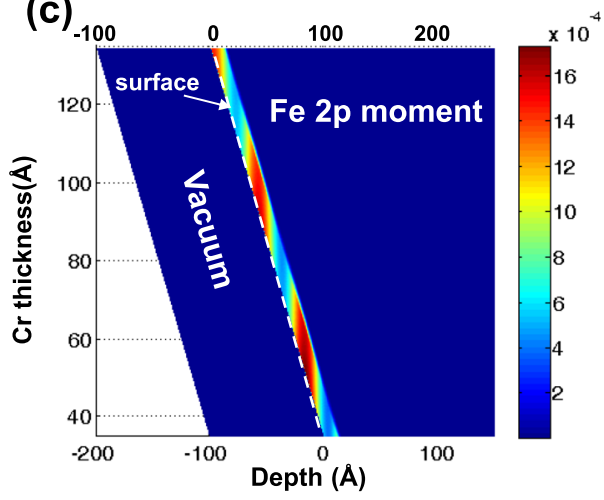

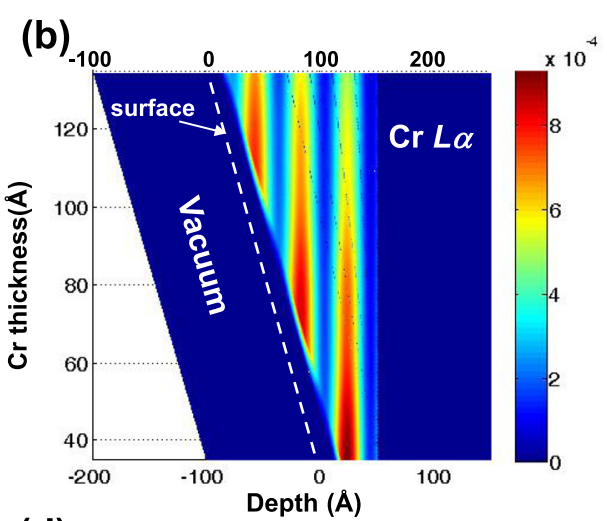

(d)

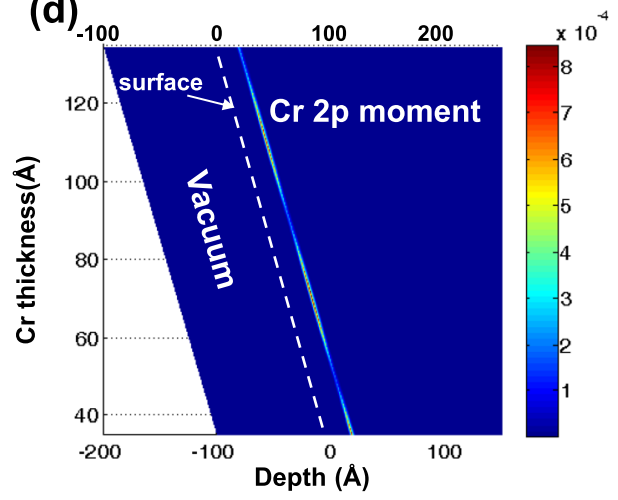

FIG. 13. Calculation of x-ray emission for a wedge-profile sample on top of the same multilayer mirror consisting of $15 \AA \mathrm{Fe} / 35 \sim 135 \AA$ Cr-wedge/[20 $\AA$ $\mathrm{B} 4 \mathrm{C} / 20 \AA \mathrm{W}]_{40}$ where the $\mathrm{Fe} / \mathrm{Cr}$ interface is uniform in both concentration and magnetization, and the photon energy is $850 \mathrm{eV}$. The incidence angle is fixed at $\phi_{B}=10.9^{\circ}$ : (a) $\mathrm{Fe} L \alpha$ and (b) $\mathrm{Cr}$ $L \alpha \mathrm{x}$-ray emission intensity profiles as a function of $\mathrm{Cr}$ thickness. (c) $\mathrm{Fe} L \alpha$ and (d) $\mathrm{Cr} L \alpha$ photoelectron intensity profiles that represent the contributions to the magnetic moments of (c) $\mathrm{Fe}$ and (d) $\mathrm{Cr}$ as a function of $\mathrm{Cr}$ thicknesses.

$$
I_{V B}\left(E_{\mathrm{kin}}, d_{\mathrm{Cr}}\right)=C \int_{0}^{\infty}\left|\vec{E}\left(z, d_{\mathrm{Cr}}\right)\right|^{2} e^{-z / \Lambda\left(E_{k i n}\right) \sin \phi} \tilde{D}\left(E_{\mathrm{kin}}, z\right) d z
$$

where $\mathrm{C}$ is a constant factor, $\vec{E}\left(z, d_{\mathrm{Cr}}\right)$ is the electric field at depth $z, \Lambda\left(E_{k i n}\right)$ is the EAL of the photoelectron, $\theta$ is again the internal photoelectron emission angle with respect to the surface, and $\tilde{D}\left(E_{\mathrm{kin}}, z\right)$ is the matrix-element weighted DOS at $E_{\text {kin }}$ at depth $z$ that we will deduce. The VB spans a small region and for soft $\mathrm{x}$-ray or hard X-ray excitation, it is thus a good approximation to assume that the EALs are constant over the full spectrum at the same energy. If we make the further assumption that $\tilde{D}\left(E_{\text {kin }}, z\right)$ is uniform in a given layer with index $L$, Eq. (41) can be rewritten as

$$
\begin{aligned}
I_{V B}\left(E_{\mathrm{kin}}, d_{\mathrm{Cr}}\right) & \cong C \sum_{L} \tilde{D}_{L}\left(E_{\mathrm{kin}}\right) \int_{z \in L}\left|\vec{E}\left(z, d_{\mathrm{Cr}}\right)\right|^{2} e^{-z / \Lambda\left(E_{k i n}\right) \sin \phi} d z \\
& =C \sum_{L} \tilde{D}_{L}\left(E_{\mathrm{kin}}\right) \int_{0}^{\infty} W_{L}\left(z, d_{\mathrm{Cr}}\right) d z
\end{aligned}
$$

where $\tilde{D}_{L}\left(E_{\mathrm{kin}}\right)$ is the matrix-element-weighted DOS at kinetic energy $E_{\text {kin }}$ for a uniform layer $L$ (here $=\mathrm{Fe}$, the $\mathrm{Fe} / \mathrm{Cr}$ interface region, $\mathrm{Cr}$, and $\mathrm{B}_{4} \mathrm{C}$ or $\mathrm{W}$ in the multilayer) and $W_{L}$ is the depth dependent weighting of each layer $L$ given by

$$
W_{L}\left(z, d_{\mathrm{Cr}}\right) \equiv\left\{\begin{array}{cl}
|\vec{E}(z, d \mathrm{Cr})|^{2} e^{-z / \Lambda\left(E_{k i n}\right) \sin \phi} & \text { if } z \in L \\
0 & \text { if } \mathrm{z} \notin \mathrm{L}
\end{array} .\right.
$$

The values of $W_{L}\left(z, d_{\mathrm{Cr}}\right)$ can be calculated by considering the combination of x-ray optics and the photoelectron emission process, as discussed earlier. Figs. 15(a)-15(c) plot $W_{L}$ for $L=\mathrm{Fe}, \mathrm{Fe} / \mathrm{Cr}$ interface, and $\mathrm{Cr}$ as functions of $z$ and $d_{\mathrm{Cr}}$.
Depending on each uniform layer, the phases and intensities of $W_{L}$ are clearly different from each other. Now, Eq. (42) can be reduced to

$$
I_{V B}\left(E_{\mathrm{kin}}, d_{\mathrm{Cr}}\right)=C \sum_{L} \tilde{D}_{L}\left(E_{\mathrm{kin}}\right) U_{L}\left(d_{\mathrm{Cr}}\right)
$$

where $U_{L}\left(d_{\mathrm{Cr}}\right)=\int W_{L}\left(z, d_{\mathrm{Cr}}\right) d z$, with curves of this integrated quantity being plotted in Fig. 15(d).

For a given $E_{\text {kin }}$ or binding energy $E_{\mathrm{B}}$, the number of $\tilde{D}_{L}\left(E_{\mathrm{kin}}\right)$ values to be determined is the number of $L$ 's $\equiv M$, while the number of equations (or the number of $d_{\mathrm{Cr}}$ 's) is the total number of VB spectra over the wedge scan $\equiv N$. Eq. (44) becomes over-determined when $N>M$, or underdetermined when $N<M$, respectively. To obtain a more reliable $\tilde{D}_{L}\left(E_{\text {kin }}\right), N \gg M$ is necessary. Thus, $\tilde{D}_{L}$ values can be uniquely determined by solving the overdetermined linear Eq. (44) in a least-squares manner way. There is an additional constraint on the solution that $\tilde{D}_{L}$ should be non-negative. The fitting calculations are straightforward and unequivocal with no fundamental physical parameters to be optimized. This method is very useful to deduce the matrix-elementweighted DOS especially at interfaces. Recently, Yang et al. ${ }^{30}$ showed that the SWEDGE method could be used to determine that the electronic structure of a $\mathrm{Fe} / \mathrm{MgO}$ interface region is FeO-like, and thus not only being significantly different from that of Fe but reducing the effective band gap of $\mathrm{MgO}$.

More recently, Kaiser et $a l .^{31}$ have used a similar approach based only upon rocking curve measurements in which a number $\mathrm{N}$ of VB spectra at different angles are compared to core-level intensities in order to study changes in the interface density of states near the Fermi level in a 
(a)

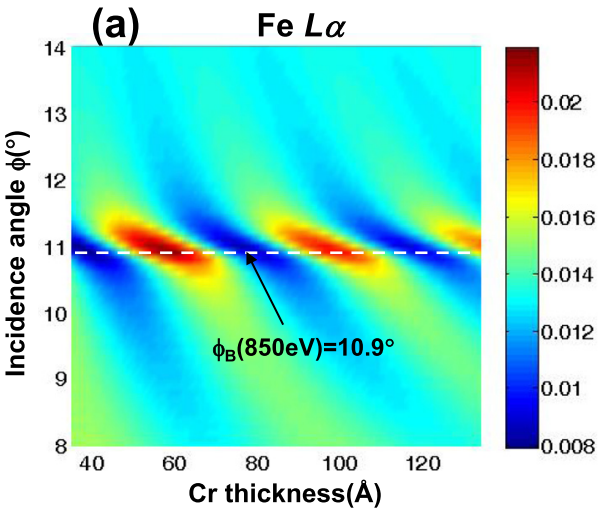

(c) Fe $L \alpha$ moment

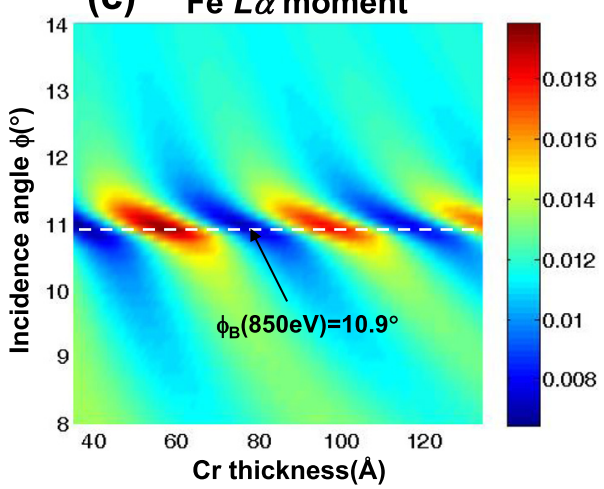

(e) Fe $L \alpha M C D$

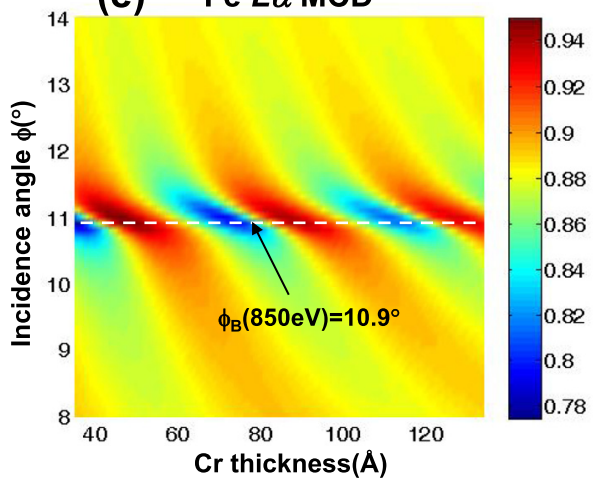

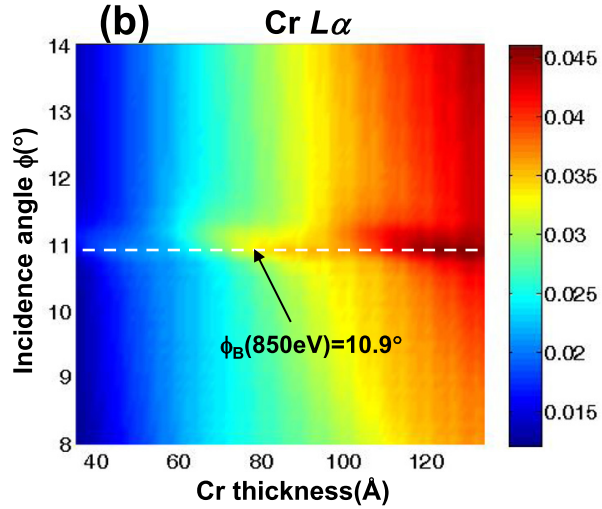

(d)

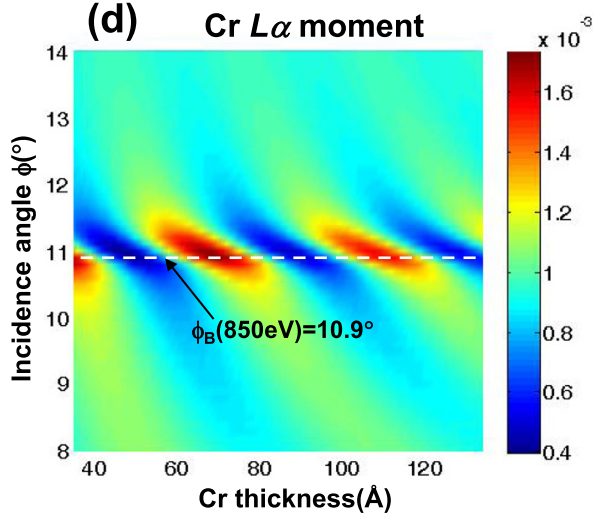

(f) $\operatorname{Cr} L \alpha M C D$

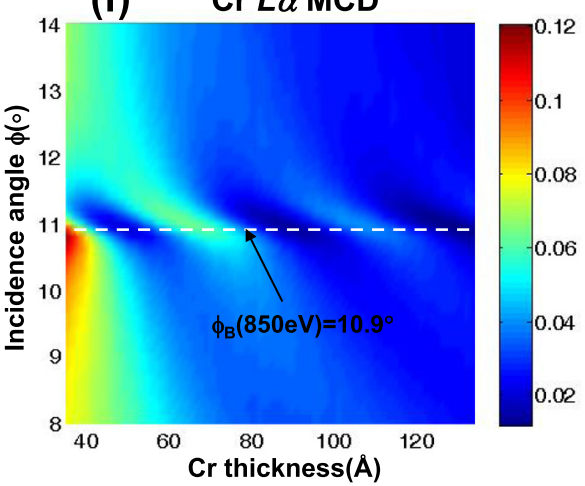

FIG. 14. (a) $\mathrm{Fe} L \alpha$ and (b) $\mathrm{Cr} L \alpha$ integrated intensity plot vs. incidence photon angle and $\mathrm{Cr}$ thicknesses based on Figs. 13(a) and 13(b) (c) Fe $L \alpha$ moment and (d) $\mathrm{Cr} L \alpha$ moment integrated intensity plot based on Figs. 13(c) and 13(d). (e) Fe $L \alpha$ MCD and (f) $\mathrm{Cr} L \alpha$ MCD contour plot. The horizontal line again indicates the Bragg angle for $850 \mathrm{eV}$.
$\mathrm{LaNiO}_{3} / \mathrm{SrTiO}_{3}$ multilayer. In this work, core-level intensities that are unique to each layer are used as the signatures of a given layer, equivalent to the $D_{L}$ parameters above, but now experimentally derived, and the VB spectra analyzed in the same sort of self-consistent least-squares analysis. This method is similar in philosophy to that used in prior standing-wave photoemission studies making use of singlecrystal planar Bragg-reflection studies. ${ }^{16}$

\section{E. Standing-wave localization, total reflection, and waveguide effects in tailored multilayer configuration}

We now consider two interesting phenomena that occur with a proper tailoring of the various layers in a multilayer sample: standing wave localization or confinement and waveguide effects. Both of these effects have the potential in certain cases of enhancing depth resolution.

A type of standing-wave localization or confinement can be achieved by employing an aperiodic multilayer with care- fully tuned optical properties and individual layer thicknesses. Fig. 16(a) is the plot of electric-field intensity $\left(E^{2}\right)$ versus sample depth for an aperiodic multilayer structure depicted in a schematic diagram in Fig. 16(b). The epitaxial multilayer consists of two types of alternating superlattice clusters. The first cluster is composed of $4 \mathrm{SrTiO}_{3} /$ $\mathrm{La}_{1.2} \mathrm{Sr}_{1.8} \mathrm{Mn}_{2} \mathrm{O}_{7}$ (STO/LSMO) bilayers where each individual layer consists of 8 unit cells. The second cluster is composed of 12 STO/LSMO bilayers where each individual layer consists of 7 unit cells. As a result of this, at each cluster boundary, the phase of the standing wave is always either delayed or advanced by $\pi$, as shown in the inset in Fig. 16(a). Therefore, at the Bragg angle, the standing-wave modulation within the sample exhibits a "beat" profile, which terminates at the surface, and almost completely confines the standing wave within the sample. The simulated reflectivity vs. angle plot in Fig. 17 exhibits a complicated structure near the Bragg angle and nears zero at the Bragg angle $\left(1.12^{\circ}\right)$ as shown in the inset, as it must for the SW to be so weak 
(a)
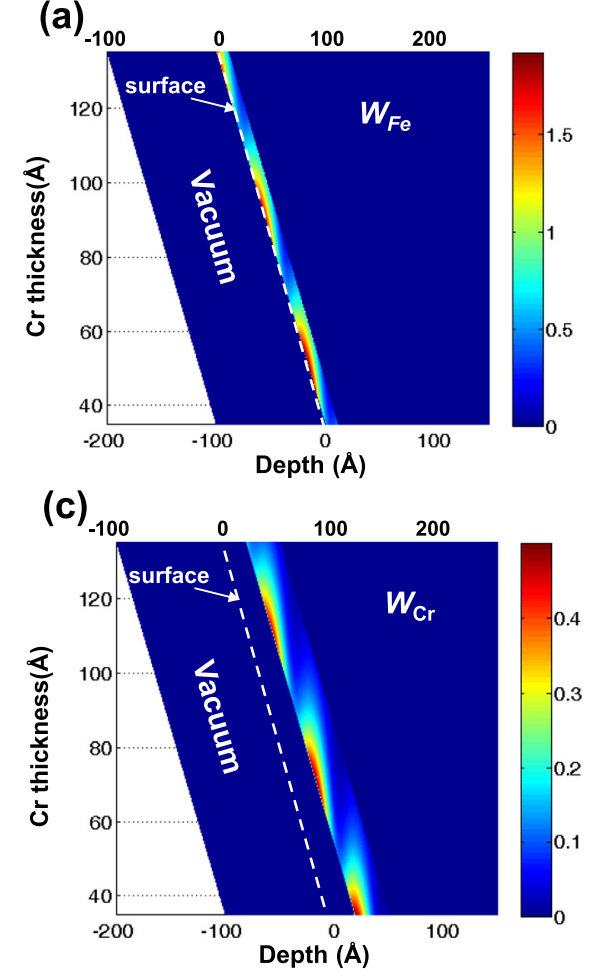

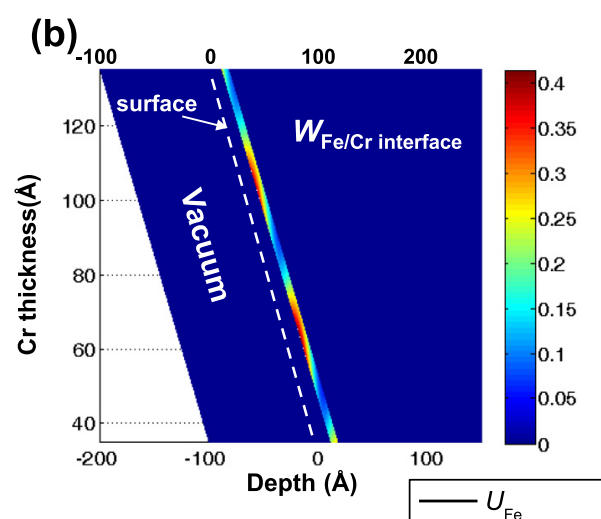

(d)

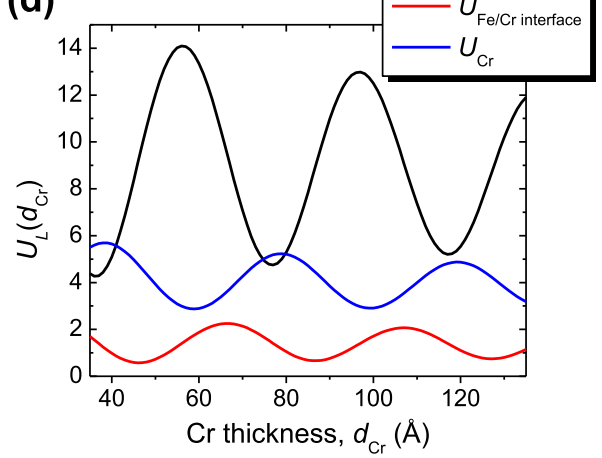

FIG. 15. Calculation steps used in deriving layer-specific densities of states for the $\mathrm{Fe} / \mathrm{Cr}$ sample of Figs. 8 and 10-14. ((a)-(c)) 3D contour plots of the depthresolved theoretical weighting factors $W_{\mathrm{L}}$ for each layer, as defined in Eq. (43), as a function of $\mathrm{Cr}$ wedge thickness $d_{\mathrm{Cr}}$ and depth $z$ for (a) $W_{\mathrm{Fe}}$, (b) $W_{\mathrm{Fe} / \mathrm{Cr} \text { interface, and }}$ (c) $W_{\mathrm{Cr}}$. Note that these include both the variation of the electric field as the SW scans through the sample and the inelastic attenuation of the escaping photoelectrons. (d) The integrals $U_{\mathrm{L}}$ of $W_{\mathrm{L}}$ over a given layer $U_{\mathrm{Fe}}$ (black), $U_{\mathrm{Fe} / \mathrm{Cr} \text { interface }}$ (red), and $U_{\mathrm{Cr}}$ (blue) are plotted versus $d_{\mathrm{Cr}}$. outside the sample. The layer configuration which leads to such a standing-wave confinement effect is by no means unique. A wide variety of aperiodic multilayers could produce this destructive interference effect above the sample surface. This phenomenon could have a wide variety of potential applications in interface science, where depthselective photoemission is of advantage. It also could potentially be applied to photoemission experiments probing solid-liquid and solid-gas interfaces, where it could be beneficial to attenuate the $\mathrm{x}$-ray electric field right above the solid sample surface so as not to sample a number of cycles of excitation in the effectively semi-infinite liquid or gas medium above the surface.

As another interesting case, we consider varying the incidence angle in the region between the Bragg angle and the region of total external reflection, a rich variety of x-ray optical phenomena, including waveguide effects, can be observed for specific film structures grown on multilayer substrates. We here for the first time also consider hard x-ray excitation at a typical energy for current experiments. ${ }^{29}$ As an example, Fig. 17(a) illustrates a schematic cross section of a sample configuration, which could be used to study interface phenomena in an exchange-bias junction. For reference, exchange bias is an effect through which an antiferromagnetic $(\mathrm{AF})$ layer (here $\mathrm{FeF}_{2}$ ) can be used to bias the magnetic hysteresis loop of an adjacent ferromagnetic (FM) layer (here $\mathrm{Co}$ ) such that this bilayer can be used as the fixed-magnetization bottom portion of a four-layer $\mathrm{AF} /$ $\mathrm{FM}$ (fixed)/metal or insulator barrier layer/FM(free) in a giant magnetoresistive or tunnel magnetoresistive structure, respectively. ${ }^{55}$ The specific model sample we have chosen consists of a GaAs/AlAs multilayer comprising 60 bilayers with a period of $44.9 \AA$, with a $200 \AA \mathrm{MgF}_{2}$ seed layer for the epitaxial growth of $\mathrm{FeF}_{2}$, followed by $100 \AA$ of $\mathrm{FeF}_{2}$ (the antiferromagnet in the exchange bias), $40 \AA$ of $\mathrm{Co}$ (the ferromagnet to be exchange biased), a $10 \AA \mathrm{Al}$ protective cap, and finally a thin layer of oxygen contaminant to simulate the surface oxidation of Al. The photoemission intensities originating from various electronic subshells are modeled as functions of $\mathrm{x}$-ray grazing incidence angle for a typical hard X-ray photon energy of $5.9 \mathrm{keV}$ in Fig. 17(b).

Typical Bragg peaks in photoemission intensities due to the constructive interference of the incident and reflected waves in the multilayer are observed near the angle of $1.4^{\circ}$ for all subshells. Most standing-wave excited photoemission experiments have been performed at or around the Bragg angle, in order to enhance the depth selectivity of the photoemission by maximizing the intensity of the standing-wave modulations. The $E$-field intensity inside the sample at the Bragg angle is plotted as a function of depth in Fig. 17(d), and it exhibits strong standing-wave modulations. On both sides of the Bragg peak, two sets of interference fringes, the Kiessig (or Fresnel) fringes mentioned previously, are observed-longer-wavelength fringes associated with the $\mathrm{Co} / \mathrm{FeF}_{2} / \mathrm{MgF}_{2}$ layers above the multilayer, and shorterwavelength fringes resulting from interference between waves reflecting from the top layer of the multilayer and from the bottom interface with the GaAs substrate. The shorter-wavelength Kiessig fringes are more evident in the blown-up plot of the region near the Bragg angle in Fig. 17(c). The separations of these fringes are fully consistent with Eq. (37) and distances D of $2688 \AA$ for the overall multilayer (compared to the input value of $2694 \AA$ ), and D of $387 \AA$ for the overlayers on the multilayer (compared to the input value of $390 \AA$ ). As the grazing incidence angle is decreased toward the onset of total external reflection at 

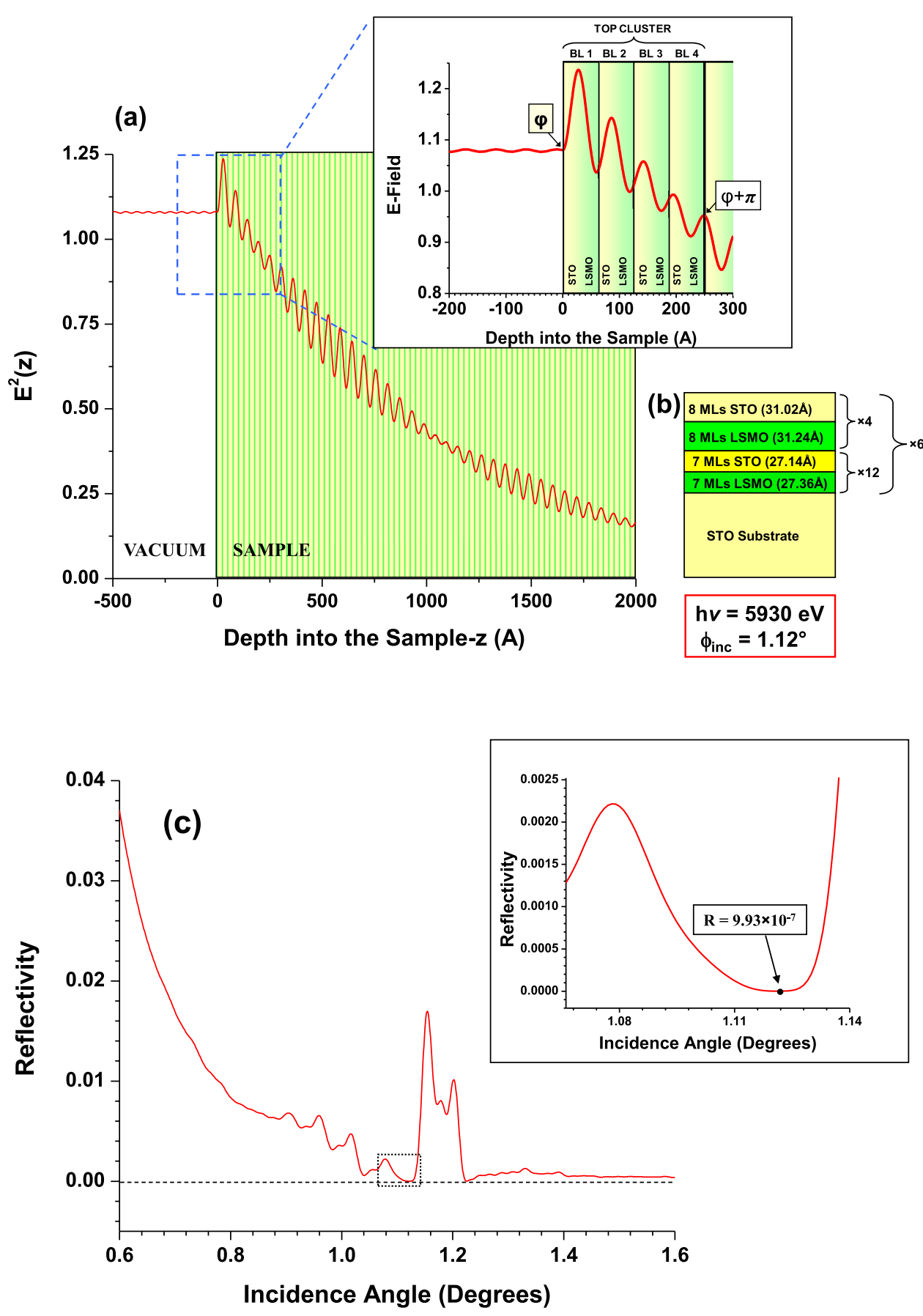

FIG. 16. (a) Calculated electric-field intensity $\left(|E|^{2}\right)$ versus sample depth for an aperiodic multilayer structure of STO and LSMO depicted in a schematic diagram in (b) and x-rays of $5930 \mathrm{eV}$ energy incident at $1.12^{\circ}$. At each cluster boundary, the phase of the standing wave is always either delayed or advanced by $\pi$, as shown by the inset in (a), and the net effect is to confine the standing-wave inside of the multilayer. (c) Calculated reflectivity vs. angle for the sample shown in (a). Note the near-zero value at an incidence angle of $1.12^{\circ}$ as shown in the inset; this must be the case, since if the standing-wave modulation is zero above the surface, the reflected wave must approach zero also. Although not shown here, it appears that an incidence angle of about $1.22^{\circ}$ should also lead to a similar standing-wave confinement, due to the very low reflectivity seen there as well. $\sim 0.4^{\circ}$, a general increase is seen in the intensities of peaks originating in the top few layers ( $\mathrm{O} 1 s$ in $\mathrm{Al}$ oxide, $\mathrm{Al} 2 p$, and $\operatorname{Co} 2 p$ ). This is due to the concentration of the E-field intensity near the surface of the sample, and is finally related at the onset of total reflection to the effect seen in Fig. 3.

As grazing angles are reduced below $\sim 0.4^{\circ}$, there is decreasing $E$-field penetration below the surface of the sample, as illustrated for point 1 in Figs. 17(b) and 17(e), and most of the photoemission originates from the $\mathrm{Al}$ oxide, $\mathrm{Al}$ and Co layers. Going to low grazing incidence angles, therefore, is an effective way of enhancing the surface sensitivity of a hard x-ray (or in fact also soft x-ray) photoemission experiment, as realized before in the technique GIXPS/ TRXPS. ${ }^{12,13}$
Finally, an unusual effect is observed at the grazing incidence angle of $0.375^{\circ}$, where the photoemission intensities originating from the $\mathrm{MgF}_{2}$ layer exhibit a very intense spike labeled as point 2. This phenomenon can be explained by examining the $E$-field intensity profile inside the sample shown in Fig. 17(f). Due to its low optical density, the $\mathrm{MgF}_{2}$ layer acts as a waveguide, enhancing the E-field intensity within itself via multiple scattering of the X-rays from the top and bottom interfaces with the adjacent $\mathrm{FeF}_{2}$ and AlAs layers, which are of higher atomic number and thus also optical density. Because of this waveguide effect, the photoemission sensitivity is enhanced dramatically inside the buried $\mathrm{MgF}_{2}$ layer. Such effects have in fact been observed in hard x-ray fluorescence experiments previously. 56 

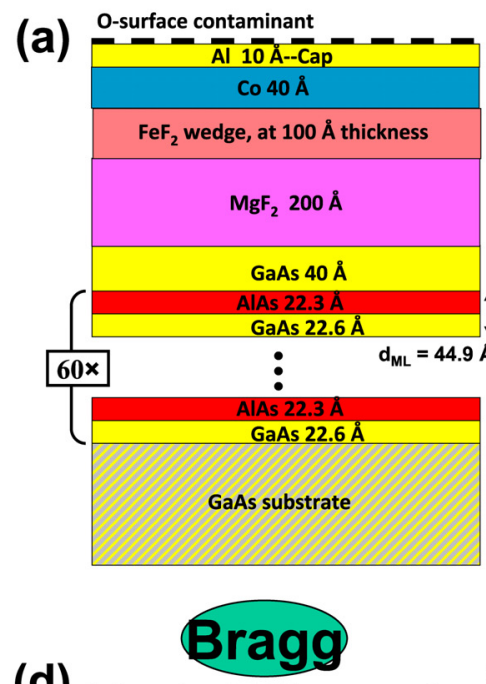

(d) [E lectric Field $]^{2}$ Vs. Depth

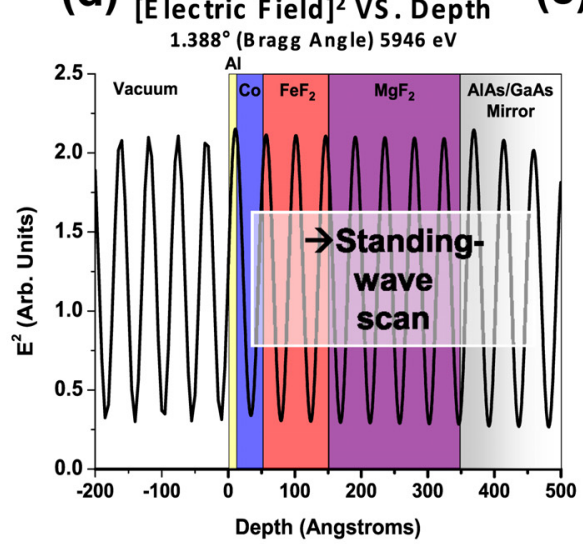

(b)

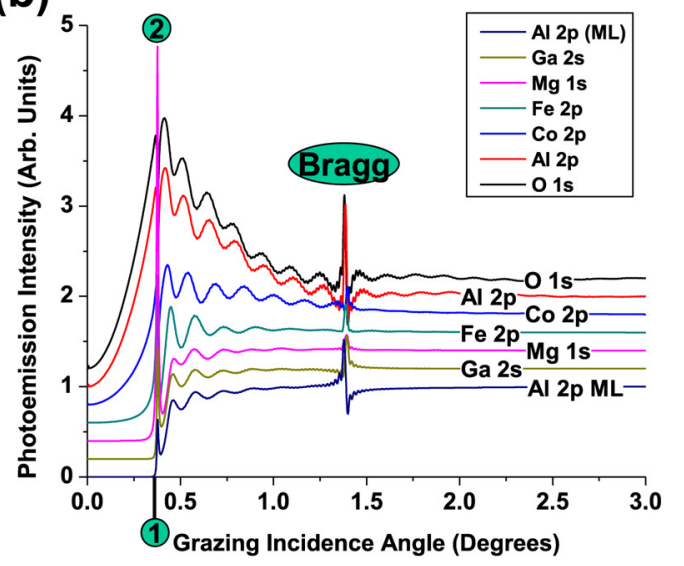

(c)
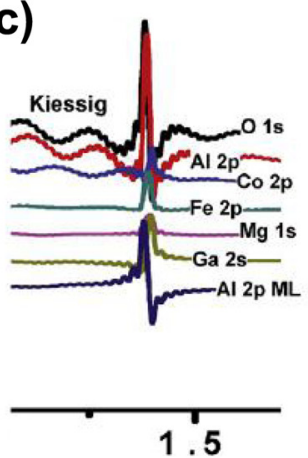

(e)

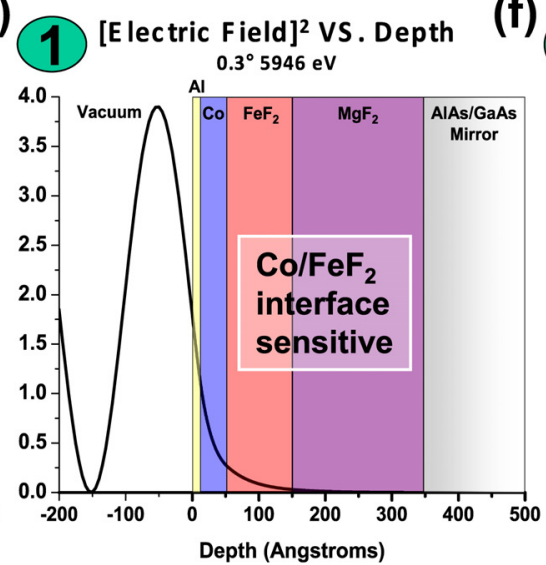

(f)

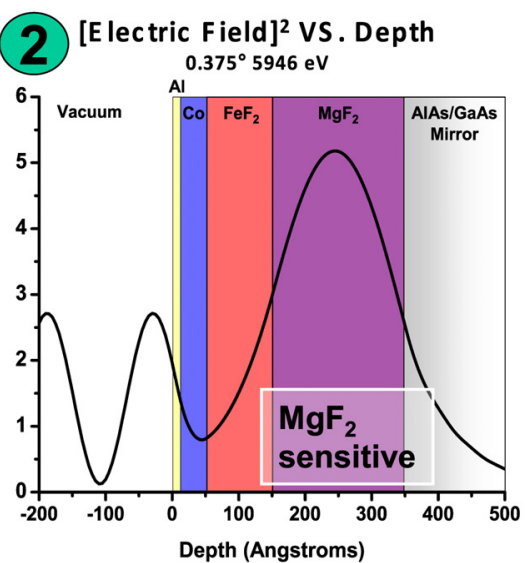

FIG. 17. (a) Schematic cross section of a hypothetical sample which could be used to study interface phenomena in an exchange-bias junction consisting of ferromagnetic $\mathrm{Co}$, antiferromagnetic $\mathrm{FeF}_{2}$ (assumed here to be $100 \AA$ thick, although it might be grown in wedge profile as needed), and a buffer layer of $\mathrm{MgF}_{2}$, all grown on a GaAs/AlAs multilayer mirror. (b) Photoemission intensities originating from various electronic subshells are calculated as a function of $\mathrm{x}$-ray grazing incidence angle for a typical hard x-ray photon energy of $5.9 \mathrm{keV}$. (c) Photoemission intensities in the region near the Bragg angle. E-field intensity as a function of depth inside the sample (d) at the Bragg angle of $1.39^{\circ}$, (e) at point 1 for a grazing incidence angle of $0.300^{\circ}$, and (f) at a grazing incidence angle of $0.375^{\circ}$, where the waveguide effect is observed in the $\mathrm{MgF}_{2}$ layer.

These two types of theoretical simulations thus suggest several ways in which $\mathrm{x}$-ray optical effects can be useful in focusing photoemission, or x-ray emission, on certain depths within a sample.

\section{F. Application to $\mathrm{nm}$-scale unit cells in layered single- crystals}

A final interesting aspect of the soft X-ray standing wave method is its application to single-crystalline systems exhibiting an intrinsically layered structure, such as the layered manganites, e.g., $\mathrm{La}_{1.2} \mathrm{Sr}_{1.8} \mathrm{Mn}_{2} \mathrm{O}_{7}$ (Ref. 57) showing colossal magnetoresistance or the high- $T_{\mathrm{c}}$ superconductors, e.g., $\mathrm{YBa}_{2} \mathrm{Cu}_{3} \mathrm{O}_{7} .{ }^{58}$ In these materials, the crystalline unit cell can be considered as a multilayer consisting of sub-planes with a different atomic constitution, e.g., $\mathrm{MnO}_{2}$ (MO) and $\mathrm{La}_{0.4} \mathrm{Sr}_{0.6} \mathrm{O}_{1}$ (LSO) planes in the layered manganite LSMO. In Sec. IV, we will concentrate on the x-ray optical properties of this material and consequences thereof.

LSMO crystallizes in the Ruddlesden-Popper phase $(\mathrm{n}=2)$ and is closely related to the perovskite $\mathrm{La}_{0.4} \mathrm{Sr}_{0.6} \mathrm{MnO}_{3}$. It can thus be modeled as depicted in Fig. 18(a). The layer thicknesses used in the simulations have been derived from the differences of the lattice parameters of the perovskite and the Ruddlesden-Popper phases and can thus only serve as a rough approximation. Uncertainty of the atomic positions has been modeled by introducing interdiffusion of the single layers of $0.5 \AA$.

Reflectivity and photoelectron intensities have been simulated for this compound, treating it as a multilayer mirror whose layers have different indices of refraction, and including an accurate experimental determination of the resonant effects on crossing the $\mathrm{Mn}-\mathrm{L}_{3}$ edge. An LSMO single crystal has been modeled using 1000 repetitions of the LSO/ $\mathrm{MO} / \mathrm{LSO} / \mathrm{MO} / \mathrm{LSO}$ pentalayers, as shown in Fig. 18(a). Fig. 18(b) shows the reflectivity of LSMO crystals for different photon energies across the $\mathrm{Mn}-\mathrm{L}_{3}$ resonance. Due to the strongly changing X-ray optical properties (see, Fig. 4(a) for the closely related $\mathrm{MnO}$ ), the shape of the Bragg peak depends on the photon energy as well. The high number of repetitions yields a reflectivity of more than $10 \%$ with a FWHM of $\sim 0.4^{\circ}$ for the resonant energy of $h \nu=634 \mathrm{eV}$. The standing wave effect is also prominent in the photoelectron yield of the $\mathrm{Sr}-3 d$ and $\mathrm{Mn}-3 p$ electrons, with overall 
(a)

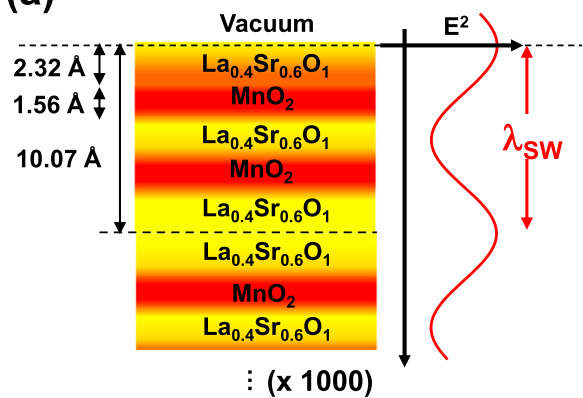

(c)

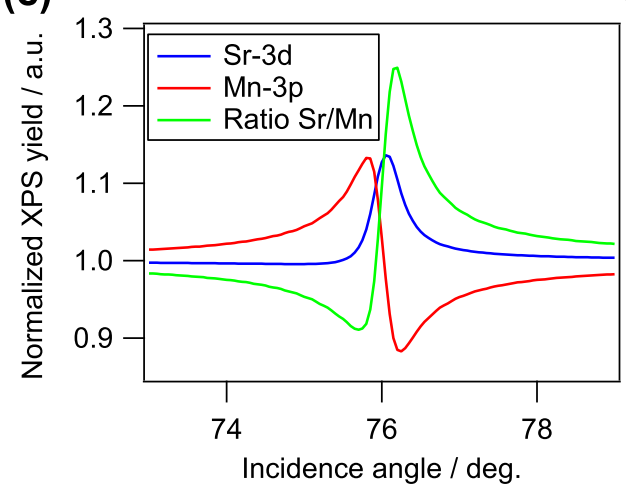

(b)

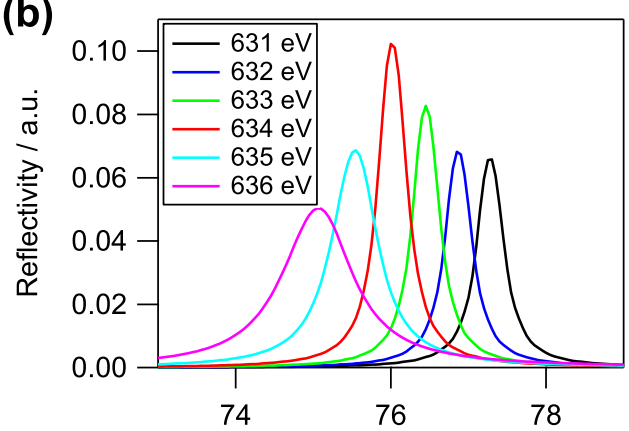

(d)

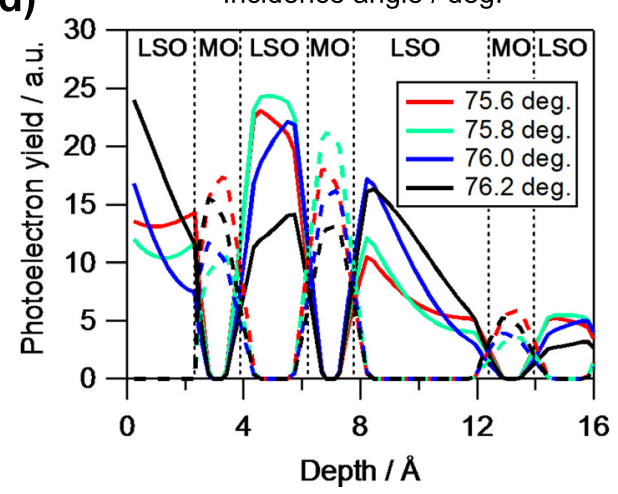

FIG. 18. (a) Schematic cross-section of a $\mathrm{La}_{1.2} \mathrm{Sr}_{1.8} \mathrm{Mn}_{2} \mathrm{O}_{7}$ bilayer manganite crystal chosen as a model system for a layered compound. The wavelength of the standing wave electric field, $\lambda_{\text {Sw }}$ (plotted on the right) spans five single layers in the compound. (b) Change of reflectivity when tuning the photon energy across the $\mathrm{Mn}-\mathrm{L}_{3}$ resonance and allowing quantitatively for the precise variation of $\delta$ and $\beta$. (c) Normalized photoelectron yields of $\mathrm{Sr}-3 d$ and $\mathrm{Mn}-3 p$ photoelectrons as a function of incidence angle near the Bragg angle. The ratio of the two yields shows a modulation of more than $30 \%$. (d) Depth-resolved photoelectron yield of Sr-3d electrons (continuous lines) and Mn-3p electrons (dashed lines) for different photon incidence angles across the Bragg peak. effects of 20\%-30\%, as shown in Fig. 18(c). The maxima and minima of the rocking curves for the two photoelectron peaks from different layers are significantly shifted relative to each other. An evaluation of the ratio of the normalized Sr-3d and Mn-3p photoelectrons for each angle reveals that one third of the overall intensity can be shifted from one layer to the other by changing the incidence angle by $0.45^{\circ}$.

However, for this particular crystal structure, it has to be noted that, unlike the case of a repeated simple nanoscale bilayers considered in prior sections, the $\mathrm{Sr}-3 d$ maximum intensity does not correspond to the $\mathrm{Mn}-3 p$ minimum intensity and vice versa. Due to the complex layered structure, one wavelength of the standing wave spans more than a single bilayer of LSO/MO, as depicted in Fig. 18(a). Therefore, in this model system, a shift of the standing wave by half a wavelength does not necessarily swap the photoelectron yield maxima of both sublayers. Nonetheless, a further analysis of the depth-resolved photoelectron yields in Fig. 18(d) shows that by varying the incidence angle, the center of gravity of the photoemission yield can be significantly moved within the wavelength of the standing wave. For example, a comparison of the photoelectron yields for $76.2^{\circ}$ and $75.8^{\circ}$ shows a significant shift of the Mn-photoemission from the first to the second sublayer and a change from surface-sensitive $\mathrm{Sr}$-photoemission to a higher sensitivity to the electrons from the buried sublayer. By carefully tuning the incidence angle and modeling the experimental data, it should thus be possible to compare the electronic structure from the surface and buried LSO layers and from different MO layers. These results thus suggest another fruitful area of application of standing-wave photoemission that can be modeled using the YXRO program. However, for such an atomic-layer specific standing-wave study, the next higher level of microscopic theory, in which the detailed atomic structure is included over many layers and each atom is described by a complex scattering factor, would be more appropriate, and would include a prediction of Bragg reflections from various crystal planes that would not be predicted by the YXRO program.

\section{SUMMARY AND OUTLOOK FOR FUTURE STUDIES}

We have presented an accurate and versatile methodology for calculating x-ray optical effects on photoelectron and Auger electron spectroscopy, as well as x-ray emission spectroscopy, including all relevant refraction, reflection, and absorption phenomena. The resulting computer program is available for general use. ${ }^{40}$ We have illustrated these $\mathrm{x}$-ray optical effects for several examples of photoelectron emission, with other applications using photoemission and $\mathrm{x}$-ray emission appearing in the references of this paper [e.g., Refs. 18, 24, and 25). Effects occurring for semi-infinite substrates have been considered, including intensity enhancements on going to grazing incidence near total reflection, without and with resonant excitation. The use of strong standing-wave effects above samples behaving in some sense like multilayer mirrors has been considered, including the possible effects of resonant excitation in studies of strongly correlated materials. The possibility of localizing standing waves near a surface by means of beat phenomena between different mirror periodicities has been suggested. The additional use of $\mathrm{x}$-ray waveguide effects for certain types of multilayer structures has also been explored. Finally, the use of standing-wave photoemission in the study of crystalline materials exhibiting a layered structure with $\mathrm{nm}$-scale periodicity in one direction has been considered. Overall, we conclude that making use of x-ray optical effects, particularly including standing waves in photoemission, as well as in 
Auger and x-ray emission, represents a versatile set of techniques for the study of various types of nanostructured materials, and that the methodology described here for modeling these effects, including the program that is openly available, permits quantitatively describing them.

\section{ACKNOWLEDGMENTS}

This work was supported by the U.S. Department of Energy, Office of Science, Office of Basic Energy Sciences, Materials Sciences Division, under Contracts No. DE-AC0376SF00098 and DE-AC02-05CH11231 and for A.M.K. also by the Army Research Office MURI Program under Award W911-NF-09-1-0398. S.-H.Y. thanks an additional support by Korea Science and Engineering Foundation (KOSEF). Research at Stanford was supported through the Stanford Institute for Materials and Energy Science (SIMES) and the LCLS by the US Department of Energy, Office of Basic Energy Sciences.

${ }^{1}$ C. J. Powell, A. Jablonski, S. Tanuma, and D. R. Penn, J. Electron Spectrosc. Relat. Phenom. 68, 605 (1994).

${ }^{2}$ C. S. Fadley, Electron Spectroscopy: Theory, Techniques, and Applications, edited by C. R. Brundle and A. D. Baker (Academic, London, 1978), Chap. 1, Vol. II.

${ }^{3}$ C. L. Briant and R. P. Messmer, Auger Electron Spectroscopy, (Academic Press, Boston, 1988).

${ }^{4} J$ J. Zegenhagen and C. Kunz, Nucl. Instrum. Methods A 547, 5 (2005).

${ }^{5}$ Programs and abstract archives from three recent hard $\mathrm{x}$-ray photoemission workshops: http://haxpes2006.spring8.or.jp/program.html; http://ssg.als. lbl.gov/ssgdirectory/fedorov/workshops/index.html; http://www.nsls.bnl.gov/ newsroom/events/workshops/2009/haxpes/.

${ }^{6}$ A. Kotani and S. Shin, Rev. Mod. Phys. 73, 203 (2001).

${ }^{7}$ X-Ray Absorption: Principles, Applications, Techniques of EXAFS, SEX$A F S$ and XANES, edited by D. C. Koningsberger and R. Prins (Wiley, 1988).

${ }^{8}$ J. B. Kortright and A. Fischer-Colbrie, J. Appl. Phys. 61, 1130 (1987).

${ }^{9}$ B. L. Henke, Phys. Rev. A 6, 94 (1972).

${ }^{10}$ M. Mehta and C. S. Fadley, Phys. Lett. A 55, 59 (1975).

${ }^{11}$ D. K. G. de Boer, Phys. Rev. B 44, 498 (1991).

${ }^{12}$ J. Chester, T. Jach, and S. Thurgate, J. Vac. Sci. Technol. B 11, 1609 (1993).

${ }^{13}$ J. Kawai, S. Hayakawa, Y. Kitajima, K. Maeda, and Y. Gohshi, J. Electron Spectrosc. Relat. Phenom. 76, 313 (1995).

${ }^{14}$ N. Mannella, S.-H. Yang, B. S. Mun, L. Zhao, A. T. Young, E. Arenholz, and C. S. Fadley, Phys. Rev. B 74, 165106 (2006).

${ }^{15}$ D. P. Woodruff, Rep. Prog. Phys. 68, 743 (2005).

${ }^{16}$ J. C. Woicik, E. J. Nelson, L. E. Berman, and I. A. Vartanyants, Phys. Rev. B 64, 125115 (2001); S. Thiess, T.-L. Lee, F. Bottin, and J. Zegenhagen, Solid State Commun. 150, 553 (2010).

${ }^{17}$ I. A. Vartanyants and M. V. Kovalchuk, Rep. Prog. Phys. 64, 1009 (2001).

${ }^{18}$ S.-H. Yang, B. S. Mun, A. W. Kay, S.-K. Kim, J. B. Kortright, J. H. Underwood, Z. Hussain, and C. S. Fadley, Surf. Sci. Lett. 461, L557 (2000).

${ }^{19}$ S. K. Kim and J. B. Kortright, Phys. Rev. Lett. 86, 1347 (2001).

${ }^{20}$ S.-H. Yang, B. S. Mun, A. W. Kay, S. K. Kim, J. B. Kortright, J. H. Underwood, Z. Hussain, and C. S. Fadley, J. Electron Spectrosc. Relat. Phenom. 114, 1089 (2001)

${ }^{21}$ S.-H. Yang, B. S. Mun, N. Mannella, S.-K. Kim, J. B. Kortright, J. H. Underwood, F. Salmassi, E. Arenholz, A. Young, Z. Hussain, M. A. Van Hove, and C. S. Fadley, J. Phys. Condens. Matter. 14, L407 (2002).

${ }^{22}$ C. S. Fadley, S.-H. Yang, B. S. Mun, and J. Garcia de Abajo, invited Chapter in the book Solid-State Photoemission and Related Methods: Theory and Experiment, edited by W. Schattke and M. A. Van Hove (Wiley-VCH Verlag, Berlin GmbH, 2003).

${ }^{23}$ S.-H. Yang, B. S. Mun, and C. S. Fadley, Synchrotron Radiat. News 17(3), 24 (2004).
${ }^{24}$ S.-H. Yang, B. C. Sell, and C. S. Fadley, J. Appl. Phys. 103, 07C519 (2008).

${ }^{25}$ S.-H. Yang, B. S. Mun, N. Mannella, A. Nambu, B. C. Sell, S. B. Ritchey, F. Salmassi, S. S. P. Parkin, and C. S. Fadley, J. Phys.: Condens. Matter 18, L259-L267 (2006).

${ }^{26}$ B. C. Sell, S. B. Ritchey, S.-H. Yang, S. S. P. Parkin, M. Watanabe, B. S. Mun, L. Plucinski, N. Mannella, A. Nambu, J. Guo, M. W. West, F. Salmassi, J. B. Kortright, and C. S. Fadley, J. Appl. Phys. 103, 083515 (2008).

${ }^{27}$ F. Kronast, R. Ovsyannikov, A. Kaiser, C. Wiemann, S. H. Yang, D. E. Burgler, R. Schreiber, F. Salmassi, P. Fischer, H. A. Dürr, C. M. Schneider, W. Eberhardt, and C. S. Fadley, Appl. Phys. Lett. 93, 243116 (2008).

${ }^{28}$ A. X. Gray, F. Kronast, C. Papp, S.-H. Yang, S. Cramm, I. P. Krug, F. Salmassi, E. M. Gullikson, D. L. Hilken, E. H. Anderson, P. Fischer, H. A. Dürr, C. M. Schneider, and C. S. Fadley, Appl. Phys. Lett. 97, 062503 (2010).

${ }^{29}$ A. X. Gray, C. Papp, B. Balke, S.-H. Yang, M. Huijben, E. Rotenberg, A. Bostwick, S. Ueda, Y. Yamashita, K. Kobayashi, E. M. Gullikson, J. B. Kortright, F. M. F. de Groot, G. Rijnders, D. H. A. Blank, R. Ramesh, and C. S. Fadley, Phys. Rev. B 82, 205116 (2010).

${ }^{30}$ S.-H. Yang, B. Balke, C. Papp, S. Döring, U. Berges, L. Plucinski, C. Westphal, C. M. Schneider, S. S. P. Parkin, and C. S. Fadley, Phys. Rev. B 84, 184410 (2011)

${ }^{31}$ A. M. Kaiser, A. X. Gray, G. Conti, J. Son, A. Greer, A. Perona, A. Rattanachata, A.Y. Saw, A. Bostwick, S. Yang, S.-H. Yang, E. M. Gullikson, J. B. Kortright, S. Stemmer, and C. S. Fadley, Phys. Rev. Lett. 107, 116402 (2011).

${ }^{32}$ C. Papp, G. Conti, B. Balke, S. Ueda, Y. Yamashita, H. Yoshikawa, Y. S. Uritsky, K. Kobayashi, and C. S. Fadley, J. Appl. Phys. 112, 114501 (2012).

${ }^{33}$ L. Z. Liu, J. W. Allen, O. Gunnarsson, N. E. Christensen, and O. K. Andersen, Phys. Rev. B 45, 8934 (1992).

${ }^{34}$ J. W. Allen and L. Z. Liu, Phys. Rev. B 46, 5047 (1992).

${ }^{35}$ A. Sekiyama, T. Iwasaki, K. Matsuda, Y. Saitoh, Y. Ônuki, and S. Suga, Nature 403, 396 (2000).

${ }^{36}$ S.-H. Yang, S.-J. Oh, H.-D. Kim, R.-J. Jung, A. Sekiyama, T. Iwasaki, S. Suga, Y. Saitoh, E.-J. Cho, and J.-G. Park, Phys. Rev. B 61, R13329 (2000).

${ }^{37}$ R.-J. Jung, B.-H. Choi, S.-J. Oh, H.-D. Kim, E.-J. Cho, T. Iwasaki, A. Sekiyama, S. Imada, S. Suga, and J.-G. Park, Phys. Rev. Lett. 91, 157601 (2003).

${ }^{38}$ S. S. P. Parkin, N. More, and K. P. Roche, Phys. Rev. Lett. 64, 2304 (1990).

${ }^{39}$ P. P. Naulleau, E. H. Anderson, E. M. Gullikson, and J. Bokor, Opt. Commun. 200, 27 (2001).

${ }^{40}$ See https://sites.google.com/a/lbl.gov/yxro/ for the downloadable YXRO computer program and the installation instructions.

${ }^{41}$ M.-R. Lefevere and M. Montel, C. R. Acad. Sci., B 273, 329 (1971).

${ }^{42}$ L. G. Parratt, Phys. Rev. 95, 359 (1954).

${ }^{43}$ C. S. Fadley, Prog. Surf. Sci. 16, 275 (1984).

${ }^{44}$ See http://eaps4.iap.tuwien.ac.at/ werner/sessa.html for the downloadable SESSA simulation computer package and instructions.

${ }^{45} \mathrm{See}$ http://www.nist.gov/srd/nist71.cfm for the NIST standard reference database of electron inelastic mean free paths.

${ }^{46}$ B. L. Henke, E. M. Gullikson, and J. C. Davis, At. Data Nucl. Data Tables 54, 181 (1993).

${ }^{47}$ J. D. Jackson, Classical Electrodynamics, 2nd ed. (Wiley, New York, 1975).

${ }^{48}$ S. Tanuma, C. J. Powell, and D. R. Penn, Surf. Interface Anal. 43, 689 (2011).

${ }^{49} \mathrm{R}$. W. James, The Optical Principles of the Diffraction of X-rays II (G. Bell and Sons, 1962).

${ }^{50}$ D. L. Windt, Comput. Phys. 12, 360 (1998).

${ }^{51}$ The Center for X-Ray Optics, X-Ray Interaction with Matter, http://henke.lbl.gov/optical_constants/.

${ }^{52}$ J. Kawai, S. Hayakawa, S. Suzuki, Y. Kitajima, Y. Takata, T. Urai, K. Meaeda, M. Fujinami, Y. Hashiguchi, and Y. Gohshi, Appl. Phys. Lett. 63, 269 (1993).

${ }^{53}$ A. Kay, E. Arenholz, B. S. Mun, F. J. Garcia de Abajo, C. S. Fadley, R. Denecke, Z. Hussain, and M. A. Van Hove, Science 281, 679 (1998).

${ }^{54}$ A. W. Kay, F. J. Garcia de Abajo, S.-H. Yang, E. Arenholz, B. S. Mun, N. Mannella, Z. Hussain, M. A. Van Hove, and C. S. Fadley, Phys. Rev. B 63, $115119(2001)$

${ }^{55}$ J. Nogues and I. K. Schuller, J. Magn. Magn. Matter. 192, 203 (1999).

${ }^{56}$ A. Gupta, N. Darowski, I. Zizak, C. Meneghini, G. Schumacher, and A. Erko, Spectrochim. Acta 62, 622 (2007).

${ }^{57}$ Y. Moritomo, A. Asamitsu, H. Kuwahara, and Y. Tokura, Nature 380, 141 (1996)

${ }^{58}$ J. D. Jorgensen, B. W. Veal, A. P. Paulikas, L. J. Nowicki, G. W. Crabtree, H. Claus, and W. K. Kwok, Phys. Rev. B 41, 1863 (1990). 